\title{
A MICROSCOPIC APPROACH TO SOUSLIN-TREE CONSTRUCTION, PART II
}

\author{
ARI MEIR BRODSKY AND ASSAF RINOT
}

\begin{abstract}
In Part I of this series, we presented the microscopic approach to Souslin-tree constructions, and argued that all known $\diamond$-based constructions of Souslin trees with various additional properties may be rendered as applications of our approach. In this paper, we show that constructions following the same approach may be carried out even in the absence of $\diamond$. In particular, we obtain a new weak sufficient condition for the existence of Souslin trees at the level of a strongly inaccessible cardinal.

We also present a new construction of a Souslin tree with an ascent path, thereby increasing the consistency strength of such a tree's nonexistence from a Mahlo cardinal to a weakly compact cardinal.

Section 2 of this paper is targeted at newcomers with minimal background. It offers a comprehensive exposition of the subject of constructing Souslin trees and the challenges involved.
\end{abstract}

\section{Contents}

1. Introduction

1.1. Two results of particular interest

1.2. Conventions

1.3. Notation

2. How to construct a Souslin tree the right way

2.1. Trees

2.2. Souslin trees

2.3. Streamlined trees

2.4. Completing canonical branches and sealing antichains

2.5. Elementary submodels and diamonds

2.6. Coherent ladder systems

2.7. A simple construction

3. Interlude: The principle

4. A generalization of $\otimes^{-}(\kappa)$

4.1. Ladder systems

4.2. Ladder systems with hitting features

4.3. Jensen's classical result and nonreflecting sets

4.4. Hitting on a club

4.5. Improving the coherence

4.6. Indexed ladders

5. Departing from $\diamond$

6. Tree constructions

6.1. Basic characteristics of trees

6.2. The underlying setup

6.3. The prototype construction

6.4. A tree with an ascent path

6.5. Omitting an ascending path

6.6. Rigid 
6.7. Free

6.8. Uniformly homogeneous and uniformly coherent

7. Some open problems

8. Acknowledgements

References

\section{INTRODUCTION}

The systematic study of set-theoretic trees was pioneered by Đuro Kurepa in the 1930s Kur35, in the context of examining Souslin's Problem. Souslin's Problem goes back a century, to 1920 [Sou20], and its most succinct formulation is:

Is every linearly ordered topological space satisfying the countable chain condition (ccc) necessarily separable?

A counterexample would be called a Souslin line 11 while the conjecture that the answer is "yes" (meaning that a Souslin line does not exist) has come to be called Souslin's Hypothesis (SH).

In the course of attempting to prove SH, Kurepa showed in 1935 Kur35 that the problem can be reformulated in terms of trees and thus "eliminated topological considerations from Souslin's Problem and reduced it to a problem of combinatorial set theory" [Kan11, p. 3]. Kurepa's result is that the existence of a Souslin line is equivalent to the existence of (what we now call) an $\aleph_{1}$-Souslin tree, that is, a tree of size $\aleph_{1}$ that includes neither an uncountable branch nor an uncountable antichain 3

Further progress toward resolving Souslin's problem came only in the 1960s, after the advent of the forcing technique, when it became apparent that Souslin's problem (at the level of $\aleph_{1}$ ) is independent of ZFC: Jech [Jec67, Tennenbaum Ten68, and Jensen Jen68, gave consistent constructions of $\aleph_{1}$-Souslin trees, while Solovay and Tennenbaum ST71] proved the consistency of SH. Amazingly enough, the resolution of this single problem led to key discoveries in set theory: various notions of trees Kur35], forcing axioms and the method of iterated forcing [ST71, the diamond and square principles [Jen72, §5-6], and the theory of iteration without adding reals DJ74, Chapter VIII].

Most of the early work around Souslin's problem focused on the level of $\aleph_{1}$, and even to this day, most of the standard references in set theory, including Dra74, Kun80, [Tod84], Roi90, JW97, HJ99], Lev02, provide a construction of a $\kappa$-Souslin tree only for the case $\kappa=\aleph_{1}$. However, Souslin's problem admits a natural generalization to higher cardinals. Indeed, Kurepa proved the following more general equivalence:

\footnotetext{
${ }^{1}$ If we remove the constraint that the topology be induced by a linear order, then there is no difficulty in obtaining a counterexample, such as the countable complement topology on any uncountable set [SS78, Counterexample 20].

${ }^{2}$ Kurepa states the equivalence between the topological and tree-based formulations of Souslin's Problem (at the level of $\aleph_{1}$ ) explicitly in Kur35, §12.D.2, pp. 124-125] Kur96 p. 111] and Kur38, Section 8, p. 134] [Kur96 p. 119]. Several sources [Rud69, p. 1116], [ST71, Section 2.1, p. 202], [DJ74 p. 12], Mal96, §2, p. 421] attribute the reformulation to E. W. Miller in 1943 [Mil43], perhaps because Kurepa's thesis and early papers were written in French. Others (Alv99, p. 213], Kan11, p. 3], Todorcevic in Kur96 p. 9]) acknowledge that Miller rediscovered Kurepa's result.

${ }^{3}$ Detailed definitions will be given in Section 2
} 
Fact 1.1 (Kurepa, Kur35]). For any regular uncountable cardinal $\kappa$, the following are equivalent 4

- Every tree of size $\kappa$ contains either a branch of size $\kappa$ or an antichain of size $\kappa$ (that is, there is no $\kappa$-Souslin tree);

- Every linearly ordered topological space satisfying the $\kappa$-chain condition $(\kappa-c c)$ has a dense subset of cardinality $<\kappa$.

The preceding leads to the following definition.

Definition 1.2 (Jen72, p. 292]). For any regular uncountable cardinal $\kappa$, the $\kappa$-Souslin hypothesis $\left(\mathrm{SH}_{\kappa}\right)$ asserts that there are no $\kappa$-Souslin trees.

Jensen proved Jen72, Theorem 6.2] that, assuming $V=L$, for every regular uncountable cardinal $\kappa, \mathrm{SH}_{\kappa}$ holds iff $\kappa$ is weakly compact. Subsequently, many combinatorial constructions of $\kappa$-Souslin trees (for regular uncountable cardinals $\kappa$ that are not weakly compact) from axioms weaker than $V=L$ have appeared 5 However, the classical constructions of $\kappa$-Souslin trees generally depend on the nature of $\kappa$ : that is, on whether $\kappa$ is the successor of a regular cardinal Gre76, She84a, Vel86; the successor of a singular cardinal [BS86, [Rin14, §4]; or an inaccessible cardinal She99.

Furthermore, the classical $\diamond$-based constructions all require $\diamond$ to concentrate on a nonreflecting stationary set, in order to ensure that sealing antichains doesn't prevent us from later building higher levels of the tree. Thus, classical methods cannot be applied in scenarios where all stationary sets reflect, and thus they allow inferring the consistency of only a Mahlo cardinal from GCH and the non-existence of a higher Souslin tree.

In addition, there is a zoo of consistent constructions of $\kappa$-Souslin trees satisfying additional properties, such as complete, regressive, rigid, homogeneous, specializable, non-specializable, admitting an ascent path, omitting an ascending path, free and uniformly coherent. Again, construction of a $\kappa$-Souslin tree with any desired property often depends on the nature of $\kappa$, and in some cases even depends on whether $\kappa$ is the successor of a singular cardinal of countable or of uncountable cofinality Cum97. To obtain the additional features, constructions include extensive bookkeeping, counters, timers, coding and decoding, whose particular nature makes it difficult to transfer the process from one type of cardinal to another.

What happens if we want to replace an axiom known to imply the existence of a $\kappa_{0}$-Souslin tree with strong properties by an axiom from which a plain $\kappa_{1}$-Souslin tree can be constructed? Do we have to revisit each scenario and tailor each of these particular constructions in order to derive a tree with strong properties?

In BR17a, which forms the starting point of this research project, we set out to develop new foundations that enable uniform construction of $\kappa$-Souslin trees; we introduced a single (parameterized) proxy principle from which $\kappa$-Souslin trees with various additional features can be constructed, regardless of the identity of $\kappa$. In that paper, we also built the bridge between the old and new foundations, establishing, among other things, that all known $\diamond$-based constructions of $\kappa$-Souslin trees may be redirected through this new proxy principle. There was one scenario that was not covered by that paper, namely, Jensen's construction from $\square(E)+$ $\diamond(E)$ Jen72, Theorem 6.2], and in Subsection [4.3 of the present paper, we cover it. This means that any $\kappa$-Souslin tree with additional features that will be shown

\footnotetext{
${ }^{4}$ Although Kurepa's explicit statements (referenced in footnote 2) refer specifically to the level of $\aleph_{1}$, he proves the result for arbitrary infinite cardinals via the equivalence $P_{2} \Longleftrightarrow P_{5}$ of the Fundamental Theorem in the Appendix Kur35 §C.3, pp. 132-133].

${ }^{5}$ See Dev84, Theorem IV.2.4], as well as Fact 1.2 of [Rin17] and the historical remarks preceding it.
} 
to follow from the proxy principle will automatically be known to hold in many unrelated models.

But the parameterized proxy principle gives us more:

- It suggests a way of calibrating the fineness of a particular class of Souslin trees, by pinpointing the weakest vector of parameters sufficient for the proxy principle to enable construction of a member of this class. This leads, for instance, to the understanding that uniformly coherent $>$ free $>$ specializable $>$ plain. This is explained in Section 6 below.

- It allows comparison and amplification of previous results.

In Jus01, KLY07, and Rin11b, new weak forms of $\diamond$ at the successor of a regular cardinal $\lambda$ were proposed and shown to entail the existence of $\lambda^{+}$-Souslin trees. In this project, we put all of these principles under a single umbrella by computing the corresponding vector of parameters for which the proxy principle holds in each of the previously studied configurations. From this and the constructions we presented in BR19c, it follows, for example, that the Gregory configuration Gre76] suffices for the construction of a specializable $\lambda^{+}$-Souslin tree, and the König-Larson-Yoshinobu configuration [KLY07] suffices for the construction of a free $\lambda^{+}$-Souslin tree.

Moreover, pump-up lemmas such as [BR19a, Lemma 4.9] and [BR19c, Lemma 3.8] establish that strong instances of the proxy principle may be derived from apparently weaker ones, leading, for example, to the existence of $\kappa$-Souslin trees with a maximal degree of completeness in unexpected scenarios.

- It allows the construction of various types of trees at a broader class of cardinals. To give two examples:

$\longrightarrow$ A combinatorial construction of a free $\kappa$-Souslin tree for $\kappa=\aleph_{1}$ may be found in DJ74, Theorem V.1], Tod84, Theorem 6.6] and [AS93, §2.1]. In [BR17b, $\S 6]$ and in [BR19c, §4.3], we gave new combinatorial constructions of free $\kappa$-Souslin trees, both using the proxy principle, and therefore they automatically apply to all regular uncountable cardinals $\kappa$, including successors of singular cardinals.

$\rightarrow$ A combinatorial construction of a uniformly coherent $\kappa$-Souslin tree for a successor of a regular cardinal $\kappa$ may be found in [DJ74, Theorem IV.1], Lar99, and Vel86. In BR17a, Theorem 2.5], we gave a proxy-based construction of a uniformly coherent $\kappa$-Souslin tree, and therefore it automatically applies to all regular uncountable cardinals $\kappa$, including inaccessible cardinals.

- It allows obtaining completely new types of Souslin trees.

Once we have suitable foundations, the construction of Souslin trees becomes simple, and it is then easier to carry out considerably more complex constructions. For example, in BR17b, Theorem 1.1], we gave the first example of a Souslin tree whose reduced powers behave independently of each other; starting from a combinatorial hypothesis that follows from " $V=L$ ", we constructed an ultrafilter $\mathcal{U}_{0}$ over $\aleph_{0}$ and an ultrafilter $\mathcal{U}_{1}$ over $\aleph_{1}$ such that, for every $(i, j) \in 2 \times 2$, there exists an $\aleph_{3}$-Souslin tree $T$ for which $T^{\aleph_{0}} / \mathcal{U}_{0}$ is $\aleph_{3}$-Aronszajn iff $i=1$ and $T^{\aleph_{1}} / \mathcal{U}_{1}$ is $\aleph_{3}$-Aronszajn iff $j=1$.

- It paves the way to finding completely new scenarios in which Souslin trees must exist, by finding new configurations in which an instance of the proxy principle holds. To give several examples:

$\longrightarrow$ In [BR17a, Corollary 1.20], we constructed a model of Martin's Maximum in which, for every regular cardinal $\kappa>\aleph_{2}$, a strong instance of the proxy principle at $\kappa$ (strong enough to yield a free $\kappa$-Souslin tree) holds.

$\longrightarrow$ In BR17a, Theorem 6.3], we proved that the sufficient condition of Gregory for the existence of a Souslin tree at the successor of a regular uncountable cardinal 
Gre76 yields an instance of the proxy principle, and then, in [BR19c, Corollary 3.4], this was generalized to include successors of singulars, as well.

$\longrightarrow$ In Rin17, Corollary 4.14], the second author proved that for every uncountable cardinal $\lambda, \mathrm{GCH}+\square\left(\lambda^{+}\right)$entails an instance of the proxy principle sufficient for the construction of a $\operatorname{cf}(\lambda)$-complete $\lambda^{+}$-Souslin tree. It follows that if $\mathrm{GCH}$ holds and there are no $\aleph_{2}$-Souslin trees, then $\aleph_{2}$ is a weakly compact cardinal in $L$, thus improving the lower bound obtained by Gregory 40 years earlier Gre76.

$\leadsto$ In [She84b], Shelah proved that adding a single Cohen real indirectly adds an $\aleph_{1}$-Souslin tree. In the same spirit, in BR19b, we identified a large class of notions of forcing that, assuming a GCH-type hypothesis, add a very strong instance of the proxy principle at the level of $\lambda^{+}$. This class includes (but is not limited to) notions of forcing for changing the cofinality of an inaccessible cardinal $\lambda$, such as Prikry, Magidor and Radin forcing.

- It gives rise to combinatorial constructions of $\kappa$-Souslin trees even in the absence of $\diamond$, which is something we did not anticipate, but is established in Sections 5 and 6 below.

- It even gives rise to results in other topics, such as special and non-special Aronszajn trees [BR19a, infinite graph theory [LR19] and Ramsey theory [RZ20].

1.1. Two results of particular interest. In the 1980s (see [Dev83]), Baumgartner proved that $\mathrm{GCH}+\square_{\aleph_{1}}$ entails the existence of an $\aleph_{2}$-Souslin tree with an $\omega$-ascent path (see Definition 6.9 below). A special case of Corollary 6.12 reads as follows.

Theorem A. GCH $+\square\left(\aleph_{2}\right)$ entails the existence of an $\aleph_{2}$-Souslin tree with an w-ascent path.

Remark 1.3. The significance of this improvement is that the consistency strength of the failure of $\square_{\aleph_{1}}$ is a Mahlo cardinal, whereas the consistency strength of the failure of $\square\left(\aleph_{2}\right)$ is a weakly compact cardinal.

As alluded to earlier, Jensen's construction of $\kappa$-Souslin trees in $L$ Jen72, Theorem 6.2] goes through the hypothesis that there exists a stationary subset $E \subset \kappa$ for which $\square(E)$ and $\diamond(E)$ both hold. Here, we obtain the same conclusion from weaker hypotheses, which is best seen for $\kappa$ inaccessible and $E \subseteq E_{>\omega}^{\kappa}$ :

Theorem B. Suppose that $\kappa$ is a strongly inaccessible cardinal, and there exists a sequence $\left\langle A_{\alpha} \mid \alpha \in E\right\rangle$ such that:

- $E$ is a nonreflecting stationary subset of $E_{>\omega}^{\kappa}$;

- For every $\alpha \in E, A_{\alpha}$ is a cofinal subset of $\alpha$;

- For every cofinal $B \subseteq \kappa$, there exists $\alpha \in E$ for which

$$
\left\{\delta<\alpha \mid \min \left(A_{\alpha} \backslash(\delta+1)\right) \in B\right\}
$$

is stationary in $\alpha$.

Then there exists a $\kappa$-Souslin tree.

1.2. Conventions. Throughout the paper, $\kappa$ stands for an arbitrary regular uncountable cardinal; $\theta, \lambda, \mu, \nu, \chi$ are (possibly finite) cardinals $\leq \kappa$; and $\xi, \sigma$ are ordinals $\leq \kappa$.

1.3. Notation. We let $H_{\kappa}$ denote the collection of all sets of hereditary cardinality less than $\kappa$ (cf. Kun80, IV, §6]). We let $\operatorname{Reg}(\lambda)$ denote the set of all infinite regular cardinals below $\lambda$. We say that $\kappa$ is $(<\chi)$-closed iff $\lambda^{<\chi}<\kappa$ for every $\lambda<\kappa$. Denote $E_{\theta}^{\lambda}:=\{\alpha<\lambda \mid \operatorname{cf}(\alpha)=\theta\}$, and define $E_{\neq \theta}^{\lambda}, E_{<\theta}^{\lambda}, E_{>\theta}^{\lambda}$, and $E_{\geq \theta}^{\lambda}$ in a similar fashion. Write $[\lambda]^{\theta}$ for the collection of all subsets of $\lambda$ of cardinality $\theta$, and define $[\lambda]^{<\theta}$ similarly. Write $\mathrm{CH}_{\lambda}$ for the assertion that $2^{\lambda}=\lambda^{+}$. 
Suppose that $C$ is a set of ordinals. Write $\operatorname{acc}(C):=\{\alpha \in C \mid \sup (C \cap \alpha)=$ $\alpha>0\}, \operatorname{nacc}(C):=C \backslash \operatorname{acc}(C), \operatorname{acc}^{+}(C):=\{\alpha<\sup (C) \mid \sup (C \cap \alpha)=\alpha>0\}$. In particular, $\operatorname{acc}(\kappa)$ is the set of all nonzero limit ordinals below $\kappa$. For any $j<\operatorname{otp}(C)$, denote by $C(j)$ the unique element $\delta \in C$ for which $\operatorname{otp}(C \cap \delta)=j$. Write $\operatorname{succ}_{\sigma}(C):=\{C(j+1) \mid j<\sigma \& j+1<\operatorname{otp}(C)\}$. In particular, for all $\gamma \in C$ such that $\sup (\operatorname{otp}(C \backslash \gamma)) \geq \sigma, \operatorname{succ}_{\sigma}(C \backslash \gamma)$ consists of the next $\sigma$ many successor elements of $C$ above $\gamma$.

The class of ordinals is denoted by ORD. For all $\alpha<\kappa, t: \alpha \rightarrow \kappa$, and $i<\kappa$, we denote by $t^{\curvearrowright} i$ the unique function $t^{\prime}$ extending $t$ satisfying $\operatorname{dom}\left(t^{\prime}\right)=\alpha+1$ and $t^{\prime}(\alpha)=i$.

\section{How to COnstruct a Souslin tree the Right WAY}

This section is accessible to novices with just basic background in Set Theory.

2.1. Trees. A tree is a partially ordered set $\left(T,<_{T}\right)$ with the property that, for every $t \in T$, the downward cone $t_{\downarrow}:=\left\{s \in T \mid s<_{T} t\right\}$ is well-ordered by $<_{T}$. The height of $t \in T$, denoted ht $(t)$, is the order-type of $\left(t_{\downarrow},<_{T}\right)$. Then, for any ordinal $\alpha$, the $\alpha^{\text {th }}$ level of $\left(T,<_{T}\right)$ is the set $T_{\alpha}:=\{t \in T \mid \operatorname{ht}(t)=\alpha\}$; the height of the tree $\left(T,<_{T}\right)$ is the smallest ordinal $\alpha$ such that $T_{\alpha}=\emptyset$. For $X \subseteq$ ORD, we write $T \nmid X:=\{t \in T \mid \operatorname{ht}(t) \in X\}=\bigcup_{\alpha \in X} T_{\alpha}$; in particular, if $\alpha$ is any ordinal, then the tree $T \uparrow \alpha$ has height $\leq \alpha$. For any $s, t \in T$, we say that $s$ and $t$ are comparable if $s<_{T} t$ or $t<_{T} s$ or $s=t$; otherwise they are incomparable.

There are several natural properties that the trees we construct will always satisfy. In particular, a tree $\left(T,<_{T}\right)$ is said to be:

- Hausdorff if for any limit ordinal $\alpha$ and $s, t \in T_{\alpha},\left(s_{\downarrow}=t_{\downarrow}\right) \Longrightarrow(s=t)$ 6

- normal if for any pair of ordinals $\alpha<\beta$ and every $s \in T_{\alpha}$, if $T_{\beta} \neq \emptyset$ then there exists some $t \in T_{\beta}$ such that $s<_{T} t$;

- ever-branching if, for every node $s \in T$, the upward cone $s^{\uparrow}:=\{t \in T \mid$ $\left.s<_{T} t\right\}$ is not linearly ordered by $<_{T}$.

2.2. Souslin trees. Suppose that $\left(T,<_{T}\right)$ is a tree. For any ordinal $\alpha$, we say that a subset $B \subseteq T$ is an $\alpha$-branch if $\left(B,<_{T}\right)$ is linearly ordered and $\{$ ht $(t)$ $t \in B\}=\alpha$. We say that $B \subseteq T$ is a cofinal branch if it is a $\kappa$-branch, where $\kappa$ is the height of $\left(T,<_{T}\right)$. We say that $A \subseteq T$ is an antichain if any two distinct $s, t \in A$ are incomparable.

A tree $\left(T,<_{T}\right)$ is a $\kappa$-tree whenever its height is $\kappa$ and $\left|T_{\alpha}\right|<\kappa$ for all $\alpha<\kappa 7$ A $\kappa$-Aronszajn tree is a $\kappa$-tree with no cofinal branches. A $\kappa$-Souslin tree is a $\kappa$-Aronszajn tree that has no antichains of size $\kappa$.

A significant focus of this paper is the construction of $\kappa$-Souslin trees. The most natural way to do this is to construct, recursively, a sequence $\left\langle T_{\alpha} \mid \alpha<\kappa\right\rangle$ of levels whose union will ultimately be the desired $\kappa$-Souslin tree. However, in order to ensure that the outcome tree will have neither any cofinal branches nor any antichains of size $\kappa$, we must find ways to anticipate these "global properties" of the tree when constructing each level. The following well-known lemma (cf. Kun80, Lemma II.7.4]) shows that if we ensure throughout the construction that our $\kappa$-tree is ever-branching (a "local property", which can be ensured level by level throughout the construction), then we can avoid the necessity of verifying that it has no cofinal branches.

Lemma 2.1. For any ever-branching $\kappa$-tree $\mathcal{T}$, the following are equivalent:

\footnotetext{
${ }^{6}$ As 0 is a limit ordinal, any (nonempty) Hausdorff tree is, in particular, rooted - that is, the level $T_{0}$ is a singleton, whose unique element is called the root.

${ }^{7}$ Recall that $\kappa$ denotes a regular uncountable cardinal.
} 
- $\mathcal{T}$ is $\kappa$-Souslin;

- $\mathcal{T}$ has no antichains of size $\kappa$.

Proof. The forward implication is obvious. Next, assume that $\mathcal{T}=\left(T,<_{T}\right)$ is an ever-branching $\kappa$-tree, having no antichains of size $\kappa$. Towards a contradiction, suppose that $\mathcal{T}$ admits a cofinal branch, say, $B$. As $\mathcal{T}$ is ever-branching, for every $t \in B, t^{\uparrow}$ is not linearly ordered, so that we may fix $t^{\prime} \in t^{\uparrow} \backslash B$. Recursively construct $B^{\bullet} \in[B]^{\kappa}$ such that, for any two $s<_{T} t$ both in $B^{\bullet}, \operatorname{ht}\left(s^{\prime}\right)<\operatorname{ht}(t)$. As $\operatorname{ht}(t)<\operatorname{ht}\left(t^{\prime}\right)$ for every $t \in B$, it follows that $\left\{t^{\prime} \mid t \in B^{\bullet}\right\}$ forms an antichain of size $\kappa$, contradicting our hypothesis and thereby completing the proof.

It follows that if we construct an ever-branching $\kappa$-tree, our main worry is to ensure the non-existence of large antichains. Furthermore, the following well-known fact (cf. [BR17b, Lemma 2.4]) shows that we do not lose any opportunities by insisting that the trees we construct are normal and ever-branching.

Fact 2.2. Suppose $\left(T,<_{T}\right)$ is a $\kappa$-Souslin tree. Then there is a normal and everbranching subtree which is again $\kappa$-Souslin. In fact, there is a club $C \subseteq \kappa$ such that $\left(T \uparrow C,<_{T}\right)$ is normal and ever-branching.

Thus, the existence of a $\kappa$-Souslin tree is equivalent to the existence of a normal ever-branching one. In fact, the same is true for $\kappa$-Aronszajn trees (cf. Kun80, Lemmas II.5.11-12]) 8

2.3. Streamlined trees. What will our trees $\left(T,<_{T}\right)$ look like? What are the elements of a tree, anyway?

Formally, of course, elements of a tree can be anything we choose. However, for all of the trees that we construct here, elements of the tree will be (transfinite) sequences of ordinals, and the tree-order $<_{T}$ will be the initial-sequence ordering (which is the same as ordinary proper inclusion $(\subsetneq$ ), if we view a sequence as a function). To ensure that the height of an element in the tree corresponds to the element's length as a sequence, we must ensure that our collection of sequences is closed under initial segments ("downward-closed").

In order to formalize this intuition while retaining some flexibility, we introduce the following definition (recall that for an ordinal $\alpha$ and any set $X,{ }^{\alpha} X$ denotes the set of functions from $\alpha$ to $X$, and $\left.{ }^{<\alpha} X:=\bigcup_{\beta<\alpha}{ }^{\beta} X\right)$ :

Definition 2.3. A set $T$ is a streamlined tree iff there exists some cardinal $\kappa$ such that $T \subseteq{ }^{<\kappa} H_{\kappa}$ and, for all $t \in T$ and $\beta<\operatorname{dom}(t), t \uparrow \beta \in T 9$

We shall freely use the following basic properties, whose verification is left to the reader.

Lemma 2.4. For every streamlined tree $T \subseteq{ }^{<\kappa} H_{\kappa}$ and every ordinal $\alpha$ :

- $(T, \subsetneq)$ is a Hausdorff tree in the abstract sense of Subsection [2.1.

- Assuming $T$ is nonempty, its root is the empty sequence, $\emptyset$.

- For every $t \in T, \operatorname{ht}(t)=\operatorname{dom}(t)$ and $t_{\downarrow}=\{t \uparrow \beta \mid \beta<\operatorname{dom}(t)\}$.

- $T_{\alpha}=T \cap{ }^{\alpha} H_{\kappa}$. In particular, $T\left\lceil\alpha=T \cap{ }^{<\alpha} H_{\kappa}\right.$.

- For every $t \in T$, if $\alpha<\operatorname{dom}(t)$, then $t\left\lceil\alpha\right.$ is the unique element of $t_{\downarrow} \cap T_{\alpha}$.

- Any $\alpha$-branch $B \subseteq T$ can be written as $B=\{t \uparrow \beta \mid \beta<\alpha\}$ for some function $t: \alpha \rightarrow \overline{H_{\kappa}}$.

- For all $s, t \in T, s$ and $t$ are comparable iff $s \cup t \in T$.

\footnotetext{
${ }^{8}$ In a sense, normality is exactly the portion of König's Lemma that can be salvaged at the height of an arbitrary regular cardinal, and this is what makes the problem of constructing $\kappa$ Aronszajn and $\kappa$-Souslin trees challenging.

${ }^{9}$ All of the $\kappa$-trees that we actually construct will be subsets of ${ }^{<\kappa} \kappa$, but we shall also consider the broader $T \subseteq{ }^{<\kappa} H_{\kappa}$ when analyzing derived trees in Subsection 6.7
} 
The main advantage of streamlined trees is the identification of a limit of an increasing sequence of nodes. Indeed, for any $\subsetneq$-increasing sequence $\eta$ of nodes, say, $\eta=\left\langle t_{\gamma} \mid \gamma<\beta\right\rangle$, the unique limit of this sequence, which may or may not be a member of the tree, is nothing but $\bigcup_{\gamma<\beta} t_{\gamma}$, that is, $\bigcup \operatorname{Im}(\eta)$.

It follows that when constructing a streamlined tree, for any limit nonzero ordinal $\alpha$ such that all of the previous levels $\left\langle T_{\beta} \mid \beta<\alpha\right\rangle$ have already been determined, the definition of $T_{\alpha}$ amounts to deciding which $\alpha$-branches will have their limits inserted into the tree. Equivalently, for any cofinal subset $C$ of $\alpha$, we shall have $T_{\alpha} \subseteq\left\{t \in{ }^{\alpha} H_{\kappa} \mid \forall \beta \in C\left(t \uparrow \beta \in T_{\beta}\right)\right\}$.

But are there any disadvantages here? It turns out that we lose no generality by insisting on constructing only streamlined trees:

Lemma 2.5. Suppose that $\left(X,<_{X}\right)$ is a $\kappa$-tree. Then:

(1) If $\left(X,<_{X}\right)$ is Hausdorff, then there exists a streamlined tree $T \subseteq{ }^{<\kappa} \kappa$ such that $\left(X,<_{X}\right)$ is order-isomorphic to $(T, \subsetneq)$.

(2) Regardless of whether or not $\left(X,<_{X}\right)$ is Hausdorff, there exists a streamlined tree $S \subseteq{ }^{<\kappa} \kappa$ such that $\left(X,<_{X}\right)$ is order-isomorphic to a cofinal subset of $(S, \subsetneq)$ via a level-preserving map.

Proof. As $\left|X_{\alpha}\right|<\kappa$ for all $\alpha<\kappa$, we may recursively find a sequence of injections $\left\langle\pi_{\alpha}: X_{\alpha} \rightarrow \kappa \mid \alpha<\kappa\right\rangle$ such that for all $\alpha<\beta<\kappa, \sup \left(\operatorname{Im}\left(\pi_{\alpha}\right)\right)<\min \left(\operatorname{Im}\left(\pi_{\beta}\right)\right)$. Let $\pi:=\bigcup_{\alpha<\kappa} \pi_{\alpha}$. Note that if $y, z \in X$ satisfy $y<_{X} z$, then $\pi(y)<\pi(z)$.

(1) Suppose $\left(X,<_{X}\right)$ is Hausdorff. For all $\delta<\kappa$ and $x \in X_{\delta}$, the set of ordinals

$$
\lceil x\rceil:=\left\{\pi(y) \mid y \in X, y<_{X} x\right\}
$$

has order-type $\delta$, so we may let $t_{x}: \delta \rightarrow\lceil x\rceil$ denote the order-preserving isomorphism. Evidently, $T:=\left\{t_{x} \mid x \in X\right\}$ is a streamlined tree, and $x \mapsto$ $t_{x}$ forms an isomorphism between $\left(X,<_{X}\right)$ and $(T, \subsetneq)$, where injectivity is due to the fact that $\left(X,<_{X}\right)$ is Hausdorff.

(2) For all $\delta<\kappa$ and $x \in X_{\delta}$, the set of ordinals

$$
[x]=\left\{\pi(y) \mid y \in X,\left(y<_{X} x \text { or } y=x\right)\right\}
$$

has order-type $\delta+1$, so we may let $s_{x}: \delta+1 \rightarrow[x]$ denote the orderpreserving isomorphism. Evidently, $S:=\left\{s_{x} \uparrow \beta \mid x \in X, \beta<\kappa\right\}$ is a streamlined tree, and $x \mapsto s_{x}$ forms an isomorphism between $\left(X,<_{X}\right)$ and a cofinal subset of $(S, \subsetneq)$ sending level $\delta$ to level $\delta+1$, where this time injectivity is due to the fact that we have included $\pi(x)$ in $[x]$.

Thus, the existence of a $\kappa$-Aronszajn is equivalent to the existence of a streamlined one, and the same is true for $\kappa$-Souslin trees.

Convention 2.6. We shall say that $T$ is a streamlined $\kappa$-tree if $T \subseteq{ }^{<\kappa} H_{\kappa}$ is a streamlined tree and $(T, \subsetneq)$ is a $\kappa$-tree. Furthermore, whenever we say that a streamlined tree $T$ is normal, ever-branching, Souslin, etc., we mean to refer to the tree $(T, \subsetneq)$.

Remark 2.7. Notice that any streamlined $\kappa$-tree $T$ is a subset of $H_{\kappa}$ and also has cardinality $\kappa$; thus $T$ and all of its subsets are elements of $H_{\kappa^{+}}$.

2.4. Completing canonical branches and sealing antichains. What does it take to build a $\kappa$-Souslin tree? Based on our previous discussion, we shall want to build, level by level, a normal, ever-branching, streamlined $\kappa$-tree.

When constructing the level $T_{\alpha}$, ensuring that the tree remains normal amounts to ensuring that for every $s \in T\left\lceil\alpha\right.$ we insert some $t$ into $T_{\alpha}$ satisfying $s \subsetneq t$. On the other hand, as all levels must be kept of size $<\kappa$ and as we must prevent the 
birth of large antichains, there will be a stationary subset $\Gamma \subseteq \kappa$ on which, for every $\alpha \in \Gamma, T_{\alpha}$ necessarily must be some proper subset of $\left\{t \in{ }^{\alpha} H_{\kappa} \mid \forall \beta<\alpha\left(t\left\lceil\beta \in T_{\beta}\right)\right\}\right.$.

But let us point out the challenge now arising in securing normality at the level $T_{\alpha}$, where $\alpha<\kappa$ is some nonzero limit ordinal. Suppose $x \in T \uparrow \alpha$; we must include a node $\mathbf{b}_{x}^{\alpha}$ in $T_{\alpha}$ extending $x$ (the limit of the "canonical $\alpha$-branch for $x$ "). The natural way to do so is to pick a club $C_{\alpha}$ in $\alpha$ ("a ladder climbing up to $\alpha$ "), and then recursively identify an increasing and continuous sequence $\left\langle x_{\beta} \mid \beta \in C_{\alpha}\right\rangle$ of nodes of $T\left\lceil\alpha\right.$ comparable with $x$ and satisfying $x_{\beta} \in T_{\beta}$ for all $\beta \in C_{\alpha}$. Normality up to level $\alpha$ makes the successor step of this recursion possible; however, when we reach a limit step $\beta$ (that is, $\beta \in \operatorname{acc}\left(C_{\alpha}\right)$ ), this ordinal $\beta$ may be an element of $\Gamma$, meaning that the unique limit of our partial sequence might have been excluded from $T_{\beta}$. If we are extremely unlucky, we may have constructed $T \uparrow \alpha$ to be an Aronszajn tree, with no $\alpha$-branches at all! Thus, we have to define $T_{\beta}$ for $\beta \in \Gamma$ in an educated way so as to avoid such unfortunate scenarios. In the special case where $\kappa=\lambda^{+}$for a (regular) cardinal $\lambda=\lambda^{<\lambda} 10$ one can avoid this problem by simply taking $\Gamma$ to be $E_{\lambda}^{\lambda^{+}}$and letting each ladder have order-type $\leq \lambda$. However, in the general case, there is a need for some coherent ladder system, as we shall see in Definition 2.16 below.

Recalling Lemma 2.1 we must also ensure that the resulting tree $T$ will not have any antichains of size $\kappa$. The number of candidates for antichains of size $\kappa$ is $\left|{ }^{<\kappa} H_{\kappa}\right|^{\kappa}$, which is bigger than $\kappa$, the length of our recursive construction. Put differently, there are not enough stages to take care of all of the candidates for large antichains, if we need to deal with them one at a time! In contrast, assuming $\kappa^{<\kappa}=\kappa$, the number of candidates for proper initial segments of antichains is merely $\kappa$. The upcoming lemma reduces the problem of eliminating antichains of size $\kappa$ to a problem of addressing their proper initial segments.

Definition 2.8. Suppose $T$ is a streamlined $\kappa$-tree. An antichain $A \subseteq T$ is said to be sealed at level $\alpha$ iff every element of $T_{\alpha}$ extends some element of $A$.

Lemma 2.9. Suppose $T$ is a streamlined $\kappa$-tree. Then the following are equivalent:

(1) $T$ has no antichains of size $\kappa$;

(2) For every antichain $A \subseteq T$, there is some ordinal $\alpha<\kappa$ such that $A \subseteq T\lceil\alpha$;

(3) For every maximal antichain $A \subseteq T$, there is some ordinal $\alpha<\kappa$ such that $A \cap(T \uparrow \alpha)$ is sealed at level $\alpha$.

Proof. (1) $\Longrightarrow$ (2): Let $A \subseteq T$ be any given antichain. By (1), $|A|<\kappa$, so that by regularity of $\kappa$, we obtain $\sup \{\operatorname{dom}(x) \mid x \in A\}<\kappa$, as sought.

(2) $\Longrightarrow$ (3): Given any maximal antichain $A \subseteq T$, fix $\alpha$ as in Clause (2), so that $A \subseteq T\left\lceil\alpha\right.$. Let $t \in T_{\alpha}$ be given. As $A$ is a maximal antichain, there must be some $s \in A$ comparable with $t$. But $\operatorname{dom}(s)<\alpha=\operatorname{dom}(t)$, so it follows that $t$ extends $s$.

(3) $\Longrightarrow$ (1): Using Zorn's lemma, it is easy to see that every antichain is included in a maximal antichain. Thus, it suffices to verify that $T$ has no maximal antichains of size $\kappa$.

Given any maximal antichain $A \subseteq T$, fix $\alpha$ as in Clause (3). As $T$ is a $\kappa$-tree, $\left|T_{\beta}\right|<\kappa$ for every $\beta$, so that by regularity of $\kappa$ it follows that $\mid T\left\lceil\alpha\left|=\sum_{\beta<\alpha}\right| T_{\beta} \mid<\kappa\right.$. Thus, it suffices to prove that $A \subseteq(T\lceil\alpha)$.

Consider any $u \in T \uparrow[\alpha, \kappa)$; we shall show that $u \notin A$. Let $t:=u \uparrow \alpha$, which is an element of $T_{\alpha}$. By our choice of $\alpha$ we can fix $s \in A$ with $s \subsetneq t$. Altogether, $s \subsetneq u$. As $s$ is an element of the antichain $A$ and $u$ properly extends $s$, we infer that $u \notin A$.

\footnotetext{
${ }^{10}$ Including, for example, the simple case $\kappa=\aleph_{1}$.
} 
In our discussion of the normality requirement, we already agreed that at limit levels $\alpha, T_{\alpha}$ will consist of elements $\mathbf{b}_{x}^{\alpha}$ extending nodes $x \in T \uparrow \alpha$. In order to accomplish Clause (3) of the preceding, we now need to ensure that given an antichain $A$, each $\mathbf{b}_{x}^{\alpha}$ extends some element of $A \cap(T\lceil\alpha)$. For this to be possible, every $x \in T \uparrow \alpha$ must be comparable with some element of $A \cap(T \uparrow \alpha)$, meaning that $A \cap(T \uparrow \alpha)$ must be a maximal antichain in $T \uparrow \alpha$. How do we locate ordinals at which properties of a given structure are replicated? This question prompts the introduction of elementary submodels.

2.5. Elementary submodels and diamonds. Recall that for every regular uncountable cardinal $\varkappa,\left(H_{\varkappa}, \in\right)$ models all axioms of ZFC except possibly for the power-set axiom. We shall be working extensively with elementary submodels $(\mathcal{M}, \in)$ of $\left(H_{\varkappa}, \in\right)$, though, by a slight abuse of notation, we will identify these structures with their underlying sets $\mathcal{M}$ and $H_{\varkappa}$, omitting the mention of the $\in-$ relation.

A comprehensive exposition of elementary submodels of $H_{\varkappa}$ may be found in [JW97, Chapter 24] and [HSW10, Chapter 4]. For now, we shall only need to be aware of the following corollary of the downward Löwenheim-Skolem theorem.

Fact 2.10. For every parameter $p \in H_{\kappa^{+}}$, the following set is cofinal in $\kappa$ :

$$
B(p):=\left\{\beta<\kappa \mid \exists \mathcal{M} \prec H_{\kappa^{+}}(p \in \mathcal{M} \& \mathcal{M} \cap \kappa=\beta)\right\} .
$$

Remark 2.11. It is not hard to verify that the set $B(p)$ is, in fact, a club in $\kappa$. But we shall not need that.

Given a well-founded poset $\mathbb{P}=(P, \triangleleft)$ which is a subset of $H_{\kappa}$ and $\beta \in B(\mathbb{P})$, for any $\mathcal{M} \prec H_{\kappa^{+}}$witnessing that $\beta \in B(\mathbb{P})$, the intersection $P \cap \mathcal{M}$ is a subset of $P$ that we can think of as being an initial segment of $\mathbb{P}$. The following proposition shows that this is precisely the case when $\mathbb{P}=(T, \subsetneq)$ and $T$ is a streamlined $\kappa$ tree, in which case the initial segment of $\mathbb{P}$ determined by $\mathcal{M}$ is nothing but $T \uparrow \beta$. Furthermore, global properties of $(T, \subsetneq)$ and its derivatives are reflected down to $\beta$ :

Proposition 2.12. Suppose that $T$ is a streamlined $\kappa$-tree, and $\beta \in B(T)$ as witnessed by $\mathcal{M} \prec H_{\kappa^{+}}$. Then:

(1) $T \cap \mathcal{M}=T \uparrow \beta$;

(2) If $A \subseteq T$ is a maximal antichain and $A \in \mathcal{M}$, then $A \cap \mathcal{M}=A \cap(T\lceil\beta)$ is a maximal antichain in $T \uparrow \beta$;

(3) If $f: T \rightarrow T$ is a nontrivial automorphism, and $f \in \mathcal{M}$, then $f \cap \mathcal{M}=$ $f \uparrow(T \uparrow \beta)$ is a nontrivial automorphism of $T \uparrow \beta$.

Proof. (1) For all $\alpha<\beta$, by $\alpha, T \in \mathcal{M}$, we obtain $T_{\alpha} \in \mathcal{M}$, and by $\mathcal{M} \models\left|T_{\alpha}\right|<\kappa$, we infer that $T_{\alpha} \subseteq \mathcal{M}$. So $T \uparrow \beta \subseteq \mathcal{M}$.

As $\operatorname{dom}(z) \in \mathcal{M} \cap \kappa$ for all $z \in T \cap \mathcal{M}$, we conclude that $T \cap \mathcal{M}=T \uparrow \beta$.

(2) Suppose $A \in \mathcal{M}$ is a maximal antichain in $T$. Since $H_{\kappa^{+}} \models A$ is a maximal antichain in $T$, it follows by elementarity that

$$
\mathcal{M} \models A \text { is a maximal antichain in } T \text {, }
$$

so that in fact $A \cap \mathcal{M}$ is a maximal antichain in $T \cap \mathcal{M}$. But $T \cap \mathcal{M}=T \uparrow \beta$ by Clause (1), so that also $A \cap \mathcal{M}=A \cap(T\lceil\beta)$. Altogether, we infer that $A \cap \mathcal{M}=A \cap(T \uparrow \beta)$ is a maximal antichain in $T \uparrow \beta$, as sought.

(3) Left to the reader.

It thus follows from Fact 2.10 and Proposition $2.12(2)$ that for any maximal antichain $A \subseteq T$, we can find cofinally many ordinals $\beta<\kappa$ such that $A \cap(T \uparrow \beta)$ is a maximal antichain in $T \uparrow \beta$. Coming back to our previous discussion, we see that as we build our tree, we will be able to seal maximal antichains of the form 
$A \cap(T \uparrow \beta)$, so that the challenge boils down to predicting $A \cap(T \uparrow \beta)$ for each and every maximal antichain $A$ of the eventual tree $T$. This leads us to discussing diamonds.

The combinatorial principle $\diamond(\kappa)$ was coined by Jensen in [Jen72, p. 293]. Rather than giving its original definition, we focus here on an equivalent formulation that is motivated by Fact 2.10

Fact 2.13 ([BR17a, Lemma 2.2]). $\diamond(\kappa)$ is equivalent to the existence of a sequence $\vec{A}=\left\langle A_{\beta} \mid \beta<\kappa\right\rangle$ of elements of $H_{\kappa}$ such that, for every parameter $p \in H_{\kappa^{+}}$and every subset $\Omega \subseteq H_{\kappa}$, the following set is cofinal in $\kappa$ :

$$
B(\Omega, p):=\left\{\beta<\kappa \mid \exists \mathcal{M} \prec H_{\kappa^{+}}\left(\mathcal{M} \cap \Omega=A_{\beta} \& p \in \mathcal{M} \& \mathcal{M} \cap \kappa=\beta\right)\right\} .
$$

Remark 2.14. It is not hard to verify that the cofinal set $B(\Omega, p)$ is, in fact, stationary in $\kappa$. Also note that a sequence $\vec{A}$ as above must form an enumeration (with repetition) of all elements of $H_{\kappa}$, thus witnessing the fact that $\diamond(\kappa)$ implies $\left|H_{\kappa}\right|=\kappa^{<\kappa}=\kappa$.

It follows from Fact 2.13 that $\diamond(\kappa)$ provides us a way to anticipate instances of Clause (2) of Proposition 2.12

Lemma 2.15 (cf. BR17a, Claim 2.3.2]). Suppose $\diamond(\kappa)$ holds, as witnessed by a sequence $\vec{A}=\left\langle A_{\beta} \mid \beta<\kappa\right\rangle$ as in Fact 2.13.

If $A$ is a maximal antichain in a given streamlined $\kappa$-tree $T$, then the following set is cofinal in $\kappa$ :

$$
B:=\left\{\beta<\kappa \mid A \cap(T \uparrow \beta)=A_{\beta} \text { is a maximal antichain in } T \uparrow \beta\right\} .
$$

Proof. Let $p:=\{T, A\}$ and $\Omega:=A$. Recalling Remark 2.7, we infer that $p \in H_{\kappa^{+}}$ and $\Omega \subseteq H_{\kappa}$, so that by our choice of $\vec{A}$, the corresponding set $B(\Omega, p)$ of Fact 2.13 is cofinal in $\kappa$. To see that $B(\Omega, p) \subseteq B$, consider any given $\beta \in B(\Omega, p)$, as witnessed by some $\mathcal{M} \prec H_{\kappa^{+}}$. Since $p \in \mathcal{M}$, by elementarity we infer that $T, A \in \mathcal{M}$. By Proposition 2.12 (2), we then deduce that $A \cap \mathcal{M}=A \cap(T\lceil\beta)$ is a maximal antichain in $T\left\lceil\beta\right.$. But $\mathcal{M} \cap A=\mathcal{M} \cap \Omega=A_{\beta}$ by our choice of $\mathcal{M}$, and it follows that $\beta \in B$, as sought.

Thus, when building the tree at the outset using a fixed diamond sequence $\vec{A}$, we take advantage of the fact that, for many ordinals $\beta, A_{\beta}$ will be a maximal antichain in $T \uparrow \beta$.

2.6. Coherent ladder systems. We now return to a point we alluded to earlier, in Subsection 2.4. Suppose we are building a limit level $T_{\alpha}$. For $x \in T \uparrow \alpha$, in order to construct $\mathbf{b}_{x}^{\alpha}$, the limit of the "canonical $\alpha$-branch for $x$ ", we want to identify an increasing and continuous sequence $\left\langle x_{\beta} \mid \beta \in C_{\alpha}\right\rangle$ of nodes of $T\lceil\alpha$ comparable with $x$ and satisfying $x_{\beta} \in T_{\beta}$ for all $\beta \in C_{\alpha}$. In order to continue this recursion through a limit step $\beta \in \operatorname{acc}\left(C_{\alpha}\right)$, we need to ensure that the limit of the partial sequence so-far identified was not excluded from $T_{\beta}$. We do this by insisting on a uniform method for constructing $\mathbf{b}_{x}^{\alpha}$, so that the limit of the partial sequence $\left\langle x_{\beta}\right|$ $\left.\beta \in C_{\alpha} \cap \beta\right\rangle$ is exactly $\mathbf{b}_{x}^{\beta}$, the limit of the canonical $\beta$-branch for $x$, which we would have inserted into $T_{\beta}$ when constructing that level. This insistence suggests several requirements whenever $\beta \in \operatorname{acc}\left(C_{\alpha}\right)$ :

(1) Coherence of the ladder system: $C_{\beta}=C_{\alpha} \cap \beta$;

(2) Microscopic perspective: the identification of the node $x_{\beta^{\prime}}$, for $\beta^{\prime} \in C_{\beta}$, must not depend on whether we are heading towards $\mathbf{b}_{x}^{\beta}$ or $\mathbf{b}_{x}^{\alpha}$;

(3) Smoothness: we must never exclude any $\mathbf{b}_{x}^{\beta}$ when constructing the level $T_{\beta}$. 
It should be clear that if we can comply with requirements (1)-(3) above, then we can construct a normal ever-branching $\kappa$-tree. But we must not forget the task of sealing antichains, and requirement (3) appears to conflict with the need to comply with Lemma 2.9. (3). How can this be resolved:11]

The answer lies in the subtlety of how we seal the antichains, more precisely, in how we decide which maximal antichain to seal at level $T_{\alpha}$. Constructing the level $T_{\alpha}$ will not involve consulting the set $A_{\alpha}$ given by Fact 2.13. Rather, when constructing $T_{\alpha}$, we will seal antichains that are predicted by $A_{\beta}$, for ordinals $\beta \in \operatorname{nacc}\left(C_{\alpha}\right)$. This approach respects requirements $(2)$ and $(3)$ above, but raises the concern of whether every maximal antichain will be predicted by $A_{\beta}$ for enough ordinals $\beta$ with $\beta \in \operatorname{nacc}\left(C_{\alpha}\right)$. This concern, together with requirement (1) above, motivates the following principle:

Definition 2.16 ([BR17a, Definition 1.3]) $\mathbb{\nabla}^{-}(\kappa)$ asserts the existence of a sequence $\vec{C}=\left\langle C_{\alpha} \mid \alpha<\kappa\right\rangle$ such that:

- for all $\alpha<\kappa, C_{\alpha}$ is a closed subset of $\alpha$ with $\sup \left(C_{\alpha}\right)=\sup (\alpha)$;

- for all $\alpha<\kappa$ and $\beta \in \operatorname{acc}\left(C_{\alpha}\right), C_{\beta}=C_{\alpha} \cap \beta$;

- for every cofinal $B \subseteq \kappa$, there exists an infinite ordinal $\alpha<\kappa$ such that $\sup \left(\operatorname{nacc}\left(C_{\alpha}\right) \cap B\right)=\alpha$.

Remarks 2.17. (1) The first bullet of Definition 2.16 implies that $C_{0}=\emptyset$, that $\max \left(C_{\alpha+1}\right)=\alpha$ for every $\alpha<\kappa$, and that $C_{\alpha}$ is a club in $\alpha$ for every $\alpha \in \operatorname{acc}(\kappa)$. In particular, any $\alpha$ whose existence is asserted in the last bullet must be a nonzero limit ordinal.

(2) If we omit the last bullet, or even weaken it by removing "nacc", then we can trivially build a witnessing sequence by setting $C_{\alpha}:=\alpha$ for every $\alpha<\kappa$. As we have alluded to in this section, and will see in detail in the proof of Proposition 2.18, it is the action of $\otimes^{-}(\kappa)$ at the non-accumulation points that enables the construction of a $\kappa$-Souslin tree by appropriately sealing the antichains without ruining the smoothness of the identification of canonical $\alpha$-branches throughout the construction.

(3) The last bullet implies that the sequence $\vec{C}$ is unthreadable, that is, there is no club $D \subseteq \kappa$ such that $D \cap \alpha=C_{\alpha}$ for all $\alpha \in \operatorname{acc}(D)$ (see BR17a, Lemma 3.2]). This bullet should be understood as a genericity feature of the coherent sequence (cf. [LR19, Lemma 3.20]).

(4) Notice that in our primary application of the last bullet of $\otimes^{-}(\kappa)$, as exemplified by the upcoming proof of Proposition 2.18, the set $B$ will be the set of ordinals where a maximal antichain is predicted, while the ordinal $\alpha$ will give us a level $T_{\alpha}$ at which such antichains are sealed. As we shall see, separating the set of ordinals where we predict a maximal antichain from the set of ordinals where we seal the predicted antichain will provide a great deal of flexibility.

(5) We encourage the reader who is already familiar with the diamond and club principles to verify that $\diamond\left(\omega_{1}\right) \Longrightarrow \boldsymbol{\phi}\left(\omega_{1}\right) \Longrightarrow \nabla^{-}\left(\omega_{1}\right) 12$ We also mention that, by BR17a, Corollary 1.10(5)], if $V=L$, then $\nabla^{-}(\kappa)$ holds for every (regular uncountable cardinal) $\kappa$ that is not weakly compact. In contrast, by [BR17a, Example 1.26], after Lévy collapsing a weakly compact cardinal to $\omega_{2}, \diamond\left(\omega_{2}\right)$ holds, but $\nabla^{-}\left(\omega_{2}\right)$ fails.

\footnotetext{
${ }^{11} \mathrm{~A}$ brief comparison of the classic non-smooth approach (requiring nonreflecting stationary sets) and the modern approach may be found on [BR17a p. 1965]. The smoothness of our approach is witnessed by Fact 2.20 below.

${ }^{12}$ See Section 3
} 
(6) The minus sign in the notation $\nabla^{-}(\kappa)$ is there to distinguish the latter from the stronger principle $\otimes(\kappa)$ of $[\mathrm{BR} 17 \mathrm{a}$, Definition 1.4].

2.7. A simple construction. Having developed the machinery in the preceding subsections, we are now ready to prove the following proposition.

Proposition 2.18 ([BR17a, Proposition 2.3]). Suppose that $\nabla^{-}(\kappa)+\diamond(\kappa)$ holds. Then there exists a $\kappa$-Souslin tree.

Proof. Let $\vec{C}=\left\langle C_{\alpha} \mid \alpha<\kappa\right\rangle$ be a witness to $\otimes^{-}(\kappa)$. Let $\vec{A}=\left\langle A_{\beta} \mid \beta<\kappa\right\rangle$ be given by Fact 2.13. In addition, let $\triangleleft$ be some well-ordering of $<\kappa_{2}$.

As outlined earlier, we shall recursively construct a sequence $\left\langle T_{\alpha} \mid \alpha<\kappa\right\rangle$ of levels such that $T:=\bigcup_{\alpha<\kappa} T_{\alpha}$ will form a normal, ever-branching, streamlined $\kappa$-Souslin tree. Furthermore, in this construction we shall ensure that for all $\alpha<\kappa$, $T_{\alpha}$ will be a subset of ${ }^{\alpha} 2$ of size $\leq \max \left\{\aleph_{0},|\alpha|\right\} 13$

- Of course, we begin by letting $T_{0}:=\{\emptyset\}$.

- Successor levels are where we will ensure that the tree is ever-branching. The simplest way to do that is to assign two immediate successors to every node from the previous level. That is, for every $\alpha<\kappa$, we let

$$
T_{\alpha+1}:=\left\{t^{\curvearrowright} 0, t^{\curvearrowright} 1 \mid t \in T_{\alpha}\right\} .
$$

Suppose that $\alpha \in \Gamma$, where $\Gamma:=\operatorname{acc}(\kappa)$, and that $\left\langle T_{\beta} \mid \beta<\alpha\right\rangle$ has already been defined. Recall that $T \uparrow \alpha=\bigcup_{\beta<\alpha} T_{\beta}$, and that constructing the level $T_{\alpha}$ involves deciding which $\alpha$-branches through $T\lceil\alpha$ will have their limits placed into the tree. As discussed in Subsection 2.4, we must balance the normality requirement with the need to bound the size of $T_{\alpha}$ and to seal antichains.

Normality requires that for every $x \in T \uparrow \alpha$ we include in $T_{\alpha}$ some node extending $x$. As $\alpha$ is a nonzero limit ordinal, our choice of the sequence $\vec{C}$ implies that $C_{\alpha}$ is a club in $\alpha$. Thus, relying on the fact that the tree $T \uparrow \alpha$ was constructed to be normal (in particular, it is normal at each level $T_{\beta}$ for $\beta \in C_{\alpha}$ ), and recalling that $T \uparrow C_{\alpha}=\bigcup_{\beta \in C_{\alpha}} T_{\beta}$, the idea for ensuring normality at level $T_{\alpha}$ is to attach to each node $x \in T \uparrow C_{\alpha}$ some node $\mathbf{b}_{x}^{\alpha} \in{ }^{\alpha} 2$ above it, and then let

$$
T_{\alpha}:=\left\{\mathbf{b}_{x}^{\alpha} \mid x \in T \uparrow C_{\alpha}\right\} .
$$

Let $x \in T \uparrow C_{\alpha}$ be arbitrary. We want $\mathbf{b}_{x}^{\alpha}$ to be the limit of some canonical $\alpha$-branch for $x$, that is, some $\alpha$-branch through $T \uparrow \alpha$ that contains $x$. As $\sup \left(C_{\alpha}\right)=\alpha$, it makes sense to describe $\mathbf{b}_{x}^{\alpha}$ as the $\operatorname{limit} \bigcup \operatorname{Im}\left(b_{x}^{\alpha}\right)$ of a sequence $b_{x}^{\alpha} \in \prod_{\beta \in C_{\alpha} \backslash \operatorname{dom}(x)} T_{\beta}$ such that:

- $b_{x}^{\alpha}(\operatorname{dom}(x))=x$;

- $b_{x}^{\alpha}\left(\beta^{\prime}\right) \subsetneq b_{x}^{\alpha}(\beta)$ for any pair $\beta^{\prime}<\beta$ of ordinals from $C_{\alpha} \backslash \operatorname{dom}(x)$;

- $b_{x}^{\alpha}(\beta)=\bigcup \operatorname{Im}\left(b_{x}^{\alpha} \uparrow \beta\right)$ for all $\beta \in \operatorname{acc}\left(C_{\alpha} \backslash \operatorname{dom}(x)\right)$.

We build the sequence $b_{x}^{\alpha}$ by recursion:

Let $b_{x}^{\alpha}(\operatorname{dom}(x)):=x$. Next, suppose $\beta^{-}<\beta$ are successive points of $C_{\alpha} \backslash \operatorname{dom}(x)$, and $b_{x}^{\alpha}\left(\beta^{-}\right)$has already been defined. In order to decide $b_{x}^{\alpha}(\beta)$, we consult the following set:

$$
Q_{x, \beta}^{\alpha}:=\left\{t \in T_{\beta} \mid \exists s \in A_{\beta}\left(s \cup b_{x}^{\alpha}\left(\beta^{-}\right)\right) \subsetneq t\right\} .
$$

Now, there are the two possibilities:

- If $Q_{x, \beta}^{\alpha} \neq \emptyset$, then let $b_{x}^{\alpha}(\beta)$ be its $\triangleleft$-least element.

- Otherwise, let $b_{x}^{\alpha}(\beta)$ be the $\triangleleft$-least element of $T_{\beta}$ that extends $b_{x}^{\alpha}\left(\beta^{-}\right)$. Such an element must exist, as the level $T_{\beta}$ was constructed so as to preserve normality.

\footnotetext{
${ }^{13}$ This means that $T$ will be slim, see Definition 6.3
} 
The following is obvious, and is aligned with the microscopic perspective described in requirement (2) of Subsection 2.6.

Dependencies 2.18.1. For any two consecutive points $\beta^{-}<\beta$ of $\operatorname{dom}\left(b_{x}^{\alpha}\right)$, the value of $b_{x}^{\alpha}(\beta)$ is completely determined by $b_{x}^{\alpha}\left(\beta^{-}\right), A_{\beta}$, and $T_{\beta}$.

Finally, suppose $\beta \in \operatorname{acc}\left(C_{\alpha} \backslash \operatorname{dom}(x)\right)$ and $b_{x}^{\alpha}\lceil\beta$ has already been defined. As promised, we let $b_{x}^{\alpha}(\beta):=\bigcup \operatorname{Im}\left(b_{x}^{\alpha} \uparrow \beta\right)$. It is clear that $b_{x}^{\alpha}(\beta) \in{ }^{\beta} 2$, but we need more than that:

Claim 2.18.2. $b_{x}^{\alpha}(\beta) \in T_{\beta}$.

Proof. First, note that since $\beta \in \operatorname{acc}\left(C_{\alpha}\right)$ and $\vec{C}$ is a $\nabla^{-}(\kappa)$-sequence, $C_{\alpha} \cap \beta=C_{\beta}$, so that $x \in T \uparrow C_{\beta}$. So, by the induction hypothesis $(*)_{\beta}$, we infer that $\mathbf{b}_{x}^{\beta}$ is in $T_{\beta} . \quad$ As $\mathbf{b}_{x}^{\beta}=\bigcup \operatorname{Im}\left(b_{x}^{\beta}\right)$ and $b_{x}^{\alpha}(\beta)=\bigcup \operatorname{Im}\left(b_{x}^{\alpha} \uparrow \beta\right)$, it thus suffices to prove that $b_{x}^{\beta}=b_{x}^{\alpha} \uparrow \beta$.

From $C_{\alpha} \cap \beta=C_{\beta}$, we obtain $\operatorname{dom}\left(b_{x}^{\beta}\right)=C_{\beta} \backslash \operatorname{dom}(x)=C_{\alpha} \cap \beta \backslash \operatorname{dom}(x)=$ $\operatorname{dom}\left(b_{x}^{\alpha}\right) \cap \beta$. Call the latter by $d$. Now, we prove that, for every $\delta \in d, b_{x}^{\beta}(\delta)=b_{x}^{\alpha}(\delta)$. By induction:

- Clearly, $b_{x}^{\beta}(\min (d))=x=b_{x}^{\alpha}(\min (d))$.

- Suppose $\delta^{-}<\delta$ are successive points of $d$, and $b_{x}^{\beta}\left(\delta^{-}\right)=b_{x}^{\alpha}\left(\delta^{-}\right)$. Then by Dependencies 2.18.1, also $b_{x}^{\beta}(\delta)=b_{x}^{\alpha}(\delta)$.

- For $\delta \in \operatorname{acc}(d)$ : If the sequences are identical up to $\delta$, then their limits must be identical.

This completes the definition of the sequence $b_{x}^{\alpha}$, and thus of its limit $\mathbf{b}_{x}^{\alpha}$, for each $x \in T \uparrow C_{\alpha}$. Consequently, the level $T_{\alpha}$ is defined as promised in $(*)_{\alpha}$.

Having constructed all levels of the tree, we then let $T:=\bigcup_{\alpha<\kappa} T_{\alpha}$. It is clear from the construction that $T$ is a normal, ever-branching, streamlined $\kappa$-tree. By Lemma 2.1, to prove that $T$ is $\kappa$-Souslin, it suffices to show that it has no $\kappa$-sized antichains. By Lemma 2.9, we thus fix an arbitrary maximal antichain $A \subseteq T$, and argue that there is some ordinal $\alpha<\kappa$ such that $A \cap(T\lceil\alpha)$ is sealed at level $\alpha$.

To find the sought-after ordinal $\alpha$, let

$$
B:=\left\{\beta<\kappa \mid A \cap(T \uparrow \beta)=A_{\beta} \text { is a maximal antichain in } T \uparrow \beta\right\} .
$$

By Lemma 2.15. $B$ is cofinal in $\kappa$. Thus, by our choice of the sequence $\vec{C}$, let us fix an infinite ordinal $\alpha<\kappa$ for which $\sup \left(\operatorname{nacc}\left(C_{\alpha}\right) \cap B\right)=\alpha$. Note that by Remark 2.17(1), $\alpha \in \Gamma$.

Claim 2.18.3. Every node of $T_{\alpha}$ extends some element of $A \cap(T \uparrow \alpha)$.

Proof. Let $t \in T_{\alpha}$ be arbitrary. As $\alpha \in \Gamma$, the construction of $T_{\alpha}$ entails that $t=\mathbf{b}_{x}^{\alpha}$ for some node $x \in T \uparrow C_{\alpha}$. Fix such an $x$. By our choice of $\alpha$, fix $\beta \in$ $\operatorname{nacc}\left(C_{\alpha}\right) \cap B$ above $\operatorname{dom}(x)$. Denote $\beta^{-}:=\sup \left(C_{\alpha} \cap \beta\right)$. Since $\beta \in B$, we know that $A_{\beta}=A \cap(T \uparrow \beta)$ is a maximal antichain in $T \uparrow \beta$, and hence there is some $s \in A_{\beta}$ comparable with $b_{x}^{\alpha}\left(\beta^{-}\right)$, so that by normality of the tree, $Q_{x, \beta}^{\alpha} \neq \emptyset$. It follows that we chose $b_{x}^{\alpha}(\beta)$ to extend some $s \in A_{\beta}$. Altogether,

$$
s \subsetneq b_{x}^{\alpha}(\beta) \subsetneq \bigcup_{\beta \in C_{\alpha} \backslash \operatorname{dom}(x)} b_{x}^{\alpha}(\beta)=\mathbf{b}_{x}^{\alpha}=t .
$$

This completes the proof.

Now that we have built a $\kappa$-Souslin tree from $\nabla^{-}(\kappa)+\diamond(\kappa)$, we mention various scenarios in which these hypotheses are known to be valid:

Fact 2.19. $\otimes^{-}(\kappa)+\diamond(\kappa)$ holds, assuming any of the following:

(1) $\kappa$ is a regular uncountable cardinal that is not weakly compact, and $V=L$ [BR17a, Corollary 1.10(5)]; 
(2) $\kappa=\aleph_{1}$ and $\diamond\left(\aleph_{1}\right)$ holds BR17a, Theorem 3.6];

(3) $\kappa=\lambda^{+}$for $\lambda$ uncountable, and $\square\left(\lambda^{+}\right)+\mathrm{GCH}$ holds [Rin17, Corollary 4.5];

(4) $\kappa=\lambda^{+}$for $\lambda$ uncountable, and $\square_{\lambda}+\mathrm{CH}_{\lambda}$ holds [BR17a, Corollary 3.9];

(5) $\kappa=\lambda^{+}$for $\lambda \geq \beth_{\omega}$, and $\square\left(\lambda^{+}\right)+\mathrm{CH}_{\lambda}$ holds [Rin17, Corollary 4.7].

It follows from Clause (3) of the preceding that in the Harrington-Shelah model HS85, Theorem A], $\nabla^{-}(\kappa)+\diamond(\kappa)$ holds for $\kappa=\aleph_{2}$, and, in addition, every stationary subset of $E_{\aleph_{0}}^{\aleph_{2}}$ reflects, meaning we are far away from the Gregory scenario Gre76. Furthermore, $\nabla^{-}(\kappa)+\diamond(\kappa)$ is compatible with the reflection of all stationary subsets of $\kappa$ :

Fact 2.20 ([Lam17a, Theorem 1.12]). Modulo a large cardinal hypothesis, there is a model of ZFC $+\mathrm{GCH}$ in which $\bigotimes^{-}(\kappa)+\diamond(\kappa)$ holds, and every stationary subset of $\kappa$ reflects, where $\kappa$ can be taken to be $\aleph_{\omega+1}$, or the first inaccessible cardinal.

By Proposition 2.18, we get a $\kappa$-Souslin tree uniformly in all of these scenarios!

After developing some more machinery in the next few sections, we shall return in Section 6 to construct a $\kappa$-Souslin tree from hypotheses considerably weaker than the ones here.

\section{Interlude: The \& PRINCIPle}

The following principle was introduced by Ostaszewski Ost76 for the special case $S=\kappa=\aleph_{1}$.

Definition 3.1. For a stationary set $S \subseteq \kappa$, the principle $(S)$ asserts the existence of a sequence $\left\langle X_{\delta} \mid \delta \in S\right\rangle$ such that:

(1) for every $\delta \in S \cap \operatorname{acc}(\kappa), X_{\delta}$ is a cofinal subset of $\delta$ with order-type $\operatorname{cf}(\delta)$;

(2) for every cofinal subset $X \subseteq \kappa$, the following set is stationary:

$$
\left\{\delta \in S \mid X_{\delta} \subseteq X\right\} .
$$

As $\boldsymbol{\beta}\left(\omega_{1}\right)$ entails $\nabla^{-}\left(\omega_{1}\right)$, it is worth spending some time to present some of the techniques involved in manipulating and improving the former.

Definition 3.2. For any two sets of ordinals $A$ and $B$, we say that $A$ is $B$-separated iff for every pair $\alpha<\alpha^{\prime}$ of ordinals from $A$, there exists $\beta \in B$ with $\alpha<\beta<\alpha^{\prime}$.

Lemma 3.3. For any two cofinal subsets $A, B$ of some limit nonzero ordinal $\delta$, there exists a cofinal subset $A^{\prime} \subseteq A$ such that $A^{\prime}$ is $B$-separated.

Proof. Let $\left\langle\delta_{i} \mid i<\operatorname{cf}(\delta)\right\rangle$ be a strictly increasing sequence of ordinals converging to $\delta$. Recursively construct a sequence $\left\langle\left(\alpha_{i}, \beta_{i}\right) \mid i<\operatorname{cf}(\delta)\right\rangle$ such that, for all $i<j<\operatorname{cf}(\delta)$ :

- $\alpha_{i} \in A$,

- $\beta_{i} \in B$, and

- $\delta_{i}<\alpha_{i}<\beta_{i}<\alpha_{i+1} \leq \alpha_{j}$.

Evidently, $A^{\prime}:=\left\{\alpha_{i} \mid i<\operatorname{cf}(\delta)\right\}$ is as sought.

Corollary 3.4. Suppose $S \subseteq \kappa$ is stationary. Then $\boldsymbol{中}(S)$ is equivalent to the existence of a sequence $\left\langle A_{\delta} \mid \delta \in S\right\rangle$ such that, for every cofinal subset $A \subseteq \kappa$, there exists a nonzero $\delta \in S$ such that $A_{\delta} \subseteq A \cap \delta$ and $\sup \left(A_{\delta}\right)=\delta$.

Proof. We focus on the nontrivial (that is, backward) implication. Let $\vec{A}=\left\langle A_{\delta}\right|$ $\delta \in S\rangle$ be as above. For every $\delta \in S \cap \operatorname{acc}(\kappa)$, if $A_{\delta}$ happens to be a cofinal subset of $\delta$, then let $X_{\delta}$ be a cofinal subset of $A_{\delta}$ of order-type $\operatorname{cf}(\delta)$; otherwise, let $X_{\delta}$ be an arbitrary cofinal subset of $\delta$ of order-type $\operatorname{cf}(\delta)$. For every $\delta \in S \backslash \operatorname{acc}(\kappa)$, just let $X_{\delta}:=\emptyset$. 
To see that $\left\langle X_{\delta} \mid \delta \in S\right\rangle$ is a $\mathbf{p}(S)$-sequence, fix an arbitrary cofinal subset $X \subseteq \kappa$ and a club $B \subseteq \kappa$; we must find $\delta \in S \cap B$ with $X_{\delta} \subseteq X$.

By Lemma 3.3. let $A$ be a cofinal subset of $X$ that is $B$-separated. By the choice of $\vec{A}$, let us fix a nonzero ordinal $\delta \in S$ such that $A_{\delta} \subseteq A \cap \delta$ and $\sup \left(A_{\delta}\right)=\delta$. In particular $\sup (A \cap \delta)=\delta$, and so by $B$-separation, also $\sup (B \cap \delta)=\delta$. But $B$ is closed, so that $\delta \in B$. In addition, $X_{\delta} \subseteq A_{\delta} \subseteq A \subseteq X$, as sought.

Lemma 3.5. Suppose that $\kappa^{\theta}=\kappa, S \subseteq \kappa$, and $\mathbf{m}(S)$ holds. Then there exists a matrix $\left\langle X_{\delta}^{\tau} \mid \delta \in S, \tau \leq \theta\right\rangle$ such that, for every sequence $\left\langle X^{\tau} \mid \tau \leq \theta\right\rangle$ of cofinal subsets of $\kappa$, there exist stationarily many $\delta \in S$, such that, for all $\tau \leq \theta$, $X_{\delta}^{\tau} \subseteq X^{\tau} \cap \delta$ and $\sup \left(X_{\delta}^{\tau}\right)=\delta$.

Proof. Let $\left\langle A_{\delta} \mid \delta \in S\right\rangle$ be a $\boldsymbol{\beta}(S)$-sequence. Fix an enumeration $\left\langle f_{\alpha} \mid \alpha<\kappa\right\rangle$ of ${ }^{\theta+1} \kappa$. Fix a club $D$ such that for all $\delta \in D$ and $\alpha<\delta, \sup \left(\operatorname{Im}\left(f_{\alpha}\right)\right)<\delta$. For all $\delta \in S$ and $\tau \leq \theta$, let $X_{\delta}^{\tau}:=\left\{f_{\alpha}(\tau) \mid \alpha \in A_{\delta}\right\}$. To see that $\left\langle X_{\delta}^{\tau} \mid \delta \in S, \tau \leq \theta\right\rangle$ is as sought, fix an arbitrary sequence $\left\langle X^{\tau} \mid \tau \leq \theta\right\rangle$ of cofinal subsets of $\kappa$. For every $\iota<\kappa$, let $X^{\tau}(\iota)$ denote the unique element $\gamma \in X^{\tau}$ such that $\operatorname{otp}\left(X^{\tau} \cap \gamma\right)=\iota$. Define $g: \kappa \rightarrow \kappa$ by stipulating:

$$
g(\iota):=\min \left\{\alpha<\kappa \mid \forall \tau \leq \theta\left(f_{\alpha}(\tau)=X^{\tau}(\iota)\right)\right\} .
$$

Notice that $g$ is injective, so that $\operatorname{Im}(g)$ is a cofinal subset of $\kappa$. Fix a cofinal subset $A$ of $\operatorname{Im}(g)$ such that, for any pair $\alpha<\alpha^{\prime}$ of ordinals from $A$, we have $\alpha<\min _{\tau \leq \theta} f_{\alpha^{\prime}}(\tau)$. Consider the stationary set:

$$
S^{\prime}:=\left\{\delta \in S \cap D \cap \operatorname{acc}(\kappa) \mid A_{\delta} \subseteq A\right\} .
$$

Let $\delta \in S^{\prime}$ and $\tau \leq \theta$. We claim that $X_{\delta}^{\tau} \subseteq X^{\tau} \cap \delta$ and $\sup \left(X_{\delta}^{\tau}\right)=\delta$. To see this, let $\alpha \in A_{\delta}$ be arbitrary. As $A_{\delta} \subseteq A \subseteq \operatorname{Im}(g)$ and $\alpha \in \delta \in D$, this means that $f_{\alpha}(\tau) \in X^{\tau} \cap \delta$. Finally, as $\sup \left(A_{\delta}\right)=\delta$ and $\alpha<f_{\alpha^{\prime}}(\tau)$ for any pair $\alpha<\alpha^{\prime}$ of ordinals from $A$, we infer that $\sup \left(X_{\delta}^{\tau}\right)=\delta$.

It follows from Remark 2.14 and Corollary 3.4 that $\diamond(\kappa) \Longrightarrow \boldsymbol{\beta}(\kappa)$. More generally, we have the following.

Fact 3.6 (Devlin, Ost76, p. 507]). For every stationary $S \subseteq \kappa$, the following are equivalent:

- $\diamond(S)$;

- $\boldsymbol{\beta}(S)$ and $\kappa^{<\kappa}=\kappa$.

In Dev78, Devlin proved that if $\diamond(S)$ holds for a stationary subset $S \subseteq \kappa$, then there exists a partition $\left\langle S_{\iota} \mid \iota<\kappa\right\rangle$ of $S$ such that $\diamond\left(S_{\iota}\right)$ holds for every $\iota<\kappa$. His proof makes essential use of the consequence $\kappa^{<\kappa}=\kappa$ of $\diamond(S)$. We now generalize Devlin's theorem and show that its analogue is valid for the weaker principle (even in the absence of $\kappa^{<\kappa}=\kappa$ ), along the way, giving a proof that applies to other variants of $\boldsymbol{s}$ and $\diamond$.

Theorem 3.7. Suppose that $\mathbf{\$}(S)$ holds for a stationary subset $S \subseteq \kappa$. Then there exists a partition $\left\langle S_{\iota} \mid \iota<\kappa\right\rangle$ of $S$ such that $\mathbf{p}\left(S_{\iota}\right)$ holds for every $\iota<\kappa$.

Proof. Fix a sequence $\vec{X}=\left\langle X_{\delta} \mid \delta \in S\right\rangle$ witnessing $\boldsymbol{\$}(S)$. Fix a bijection $\pi: \kappa \leftrightarrow$ $\kappa \times \kappa$. Define $h: S \rightarrow \kappa$ by letting $h(\delta):=0$ for $\delta \in S \backslash \operatorname{acc}(\kappa)$, and, for every $\delta \in S \cap \operatorname{acc}(\kappa)$,

$$
h(\delta):=\min \left\{\iota<\kappa \mid(\kappa \times\{\iota\}) \cap \pi\left[X_{\delta}\right] \neq \emptyset\right\} .
$$

For every $\iota<\kappa$, let $S_{\iota}:=\{\delta \in S \mid h(\delta)=\iota\}$, so that $\left\langle S_{\iota} \mid \iota<\kappa\right\rangle$ is a partition of $S$.

Next, for every $\delta \in S \cap \operatorname{acc}(\kappa)$, let

$$
A_{\delta}:=\left\{\gamma<\delta \mid(\gamma, h(\delta)) \in \pi\left[X_{\delta}\right]\right\},
$$

while for $\delta \in S \backslash \operatorname{acc}(\kappa)$, just let $A_{\delta}:=\emptyset$. 
Claim 3.7.1. Let $A \subseteq \kappa$ be cofinal. For every $\iota<\kappa$, there exists a nonzero $\delta \in S_{\iota}$ such that $A_{\delta} \subseteq A$ and $\sup \left(A_{\delta}\right)=\delta$.

Proof. Let $\iota<\kappa$ be arbitrary. Put $X:=\pi^{-1}[A \times\{\iota\}]$. As $\pi$ is bijective, $|X|=$ $|A|=\kappa$, so that $X$ is cofinal in $\kappa$. Consider the $\operatorname{club} B:=\{\beta<\kappa \mid \pi[\beta]=\beta \times \beta\}$. By Lemma 3.3. fix a cofinal $X^{\prime} \subseteq X$ that is $B$-separated. Now, by the choice of $\vec{X}$, the following set is stationary:

$$
G\left(X^{\prime}\right):=\left\{\delta \in S \mid X_{\delta} \subseteq X^{\prime}\right\} .
$$

Fix $\delta \in G\left(X^{\prime}\right) \cap \operatorname{acc}(\kappa)$ above $\iota$. As $X_{\delta} \subseteq X^{\prime}$, we infer $\pi\left[X_{\delta}\right] \subseteq \pi\left[X^{\prime}\right] \subseteq \pi[X]=$ $A \times\{\iota\}$, so that $h(\delta)=\iota$. It thus follows that $\delta \in S_{\iota}$ and $A_{\delta} \subseteq A$. Finally, to see that $\sup \left(A_{\delta}\right)=\delta$, let $\alpha<\delta$ be arbitrary, and we shall find $\gamma \in A_{\delta}$ above $\alpha$. As $\sup \left(X_{\delta}\right)=\delta$, we may assume that $\alpha \in X_{\delta} \backslash \iota$. Let $\alpha^{\prime}:=\min \left(X_{\delta} \backslash(\alpha+1)\right)$. As $X_{\delta} \subseteq X^{\prime}$ and the latter is $B$-separated, let us also fix $\beta \in B$ with $\alpha<\beta<\alpha^{\prime}$. Since $\pi\left[X_{\delta}\right] \subseteq A \times\{\iota\}$, we can fix $\gamma$ such that $\pi\left(\alpha^{\prime}\right)=(\gamma, \iota)$. Since $\pi[\beta]=\beta \times \beta$ while $\iota \leq \alpha<\beta<\alpha^{\prime}$, it follows that $\gamma \geq \beta$. As $\sup \left(X_{\delta}\right)=\delta$ and $X_{\delta} \subseteq X^{\prime}$, we infer that $\sup \left(X^{\prime} \cap \delta\right)=\delta$, so that by $B$-separation of $X^{\prime}$, we also obtain $\sup (B \cap \delta)=\delta$. But $B$ is closed, so that $\delta \in B$, meaning that $\pi[\delta]=\delta \times \delta$. As $\alpha^{\prime}<\delta$, it follows that $\gamma<\delta$. Altogether, $\gamma$ is an element of $A_{\delta}$ above $\alpha$.

As $A_{\delta} \subseteq A$ for all $\delta \in S$, it now follows from Corollary 3.4 that $\boldsymbol{\beta}\left(S_{\iota}\right)$ holds for all $\iota<\kappa$.

When replacing sets of ordinals by sets of sets of ordinals, it is natural to replace the sup measure by a sup-over-min measure which we call mup:

Definition 3.8. For every $\mathcal{A} \subseteq \mathcal{P}(\kappa)$, let $\operatorname{mup}(\mathcal{A}):=\sup \{\min (a) \mid a \in \mathcal{A}, a \neq \emptyset\}$.

A minor variation of the proof of Theorem 3.7 establishes yet another equivalence, which will be utilized in deriving Theorem 4.16 below.

Lemma 3.9. For every stationary $S \subseteq \kappa, \boldsymbol{中}(S)$ holds iff there exists a sequence $\left\langle\mathcal{X}_{\delta} \mid \delta \in S\right\rangle$ such that:

(1) for every $\delta \in S \cap \operatorname{acc}(\kappa), \mathcal{X}_{\delta} \subseteq[\delta]^{<\omega}$ with $\operatorname{mup}\left(\mathcal{X}_{\delta}\right)=\delta$;

(2) for every $\mathcal{X} \subseteq[\kappa]^{<\omega}$ with $\operatorname{mup}(\mathcal{X})=\kappa$, the following set is stationary:

$$
\left\{\delta \in S \mid \mathcal{X}_{\delta} \subseteq \mathcal{X}\right\} \text {. }
$$

Proof. We focus on the nontrivial (that is, forward) implication. Fix a sequence $\vec{A}=\left\langle A_{\delta} \mid \delta \in S\right\rangle$ witnessing $(S)$. Fix a bijection $\pi: \kappa \leftrightarrow[\kappa]^{<\omega}$. For every $\delta \in S$, let

$$
\mathcal{X}_{\delta}:= \begin{cases}\pi\left[A_{\delta}\right], & \text { if } \delta \in \operatorname{acc}(\kappa) \& \pi\left[A_{\delta}\right] \subseteq[\delta]^{<\omega} \& \operatorname{mup}\left(\pi\left[A_{\delta}\right]\right)=\delta ; \\ {[\delta]^{<\omega},} & \text { otherwise. }\end{cases}
$$

To see that $\left\langle\mathcal{X}_{\delta} \mid \delta \in S\right\rangle$ satisfies Clause (2) of the lemma, fix an arbitrary $\mathcal{X} \subseteq[\kappa]^{<\omega}$ with $\operatorname{mup}(\mathcal{X})=\kappa$. By thinning $\mathcal{X}$ out, we may assume that $\emptyset \notin \mathcal{X}$ and that $x \mapsto \min (x)$ is injective over $\mathcal{X}$. Now, let $A:=\pi^{-1}[\mathcal{X}]$, so that $|A|=\kappa$. Consider the following set:

$$
B:=\left\{\beta<\kappa \mid \pi[\beta]=[\beta]^{<\omega} \& \forall x \in \mathcal{X}(\min (x)<\beta \Longrightarrow \max (x)<\beta)\right\} .
$$

It is not hard to see that for $p:=\{\pi, \mathcal{X}\}$, our set $B$ covers the set $B(p)$ of Fact 2.10, so that $B$ is cofinal in $\kappa$. By Lemma 3.3, fix a cofinal $A^{\prime} \subseteq A$ that is $B$-separated. Now, by the choice of $\vec{A}$, there are stationarily many $\delta \in S \cap \operatorname{acc}(\kappa)$ for which $A_{\delta} \subseteq A^{\prime}$. Fix such a $\delta$, and we shall show that $\mathcal{X}_{\delta} \subseteq \mathcal{X}$.

As $A_{\delta} \subseteq A^{\prime}$, we infer $\pi\left[A_{\delta}\right] \subseteq \pi\left[A^{\prime}\right] \subseteq \pi[A]=\mathcal{X}$. By definition of $\mathcal{X}_{\delta}$, then, it suffices to prove that $\pi\left[A_{\delta}\right] \subseteq[\delta]^{<\omega}$ and $\operatorname{mup}\left(\pi\left[A_{\delta}\right]\right)=\delta$. To prove the former: As $\sup \left(A_{\delta}\right)=\delta$ and $A_{\delta} \subseteq A^{\prime}$, we infer that $\sup \left(A^{\prime} \cap \delta\right)=\delta$, so that by $B$-separation 
of $A^{\prime}$, we also obtain $\sup (B \cap \delta)=\delta$. But $B$ is closed, so that $\delta \in B$, and as $A_{\delta} \subseteq \delta$ we infer that $\pi\left[A_{\delta}\right] \subseteq \pi[\delta]=[\delta]^{<\omega}$. To see that $\operatorname{mup}\left(\pi\left[A_{\delta}\right]\right)=\delta$, let $\alpha<\delta$ be arbitrary, and we shall find $\alpha^{\prime} \in A_{\delta}$ with $\min \left(\pi\left(\alpha^{\prime}\right)\right)>\alpha$. As $\sup \left(A_{\delta}\right)=\delta$, we may assume that $\alpha \in A_{\delta}$. Let $\alpha^{\prime}:=\min \left(A_{\delta} \backslash(\alpha+1)\right)$. As $A_{\delta} \subseteq A^{\prime}$ and the latter is $B$-separated, let us also fix $\beta \in B$ with $\alpha<\beta<\alpha^{\prime}$. Since $\pi[\beta]=[\beta]^{<\omega}$, we know that $\max \left(\pi\left(\alpha^{\prime}\right)\right) \geq \beta$. Since $\beta \in B$ and $\pi\left(\alpha^{\prime}\right) \in \pi\left[A_{\delta}\right] \subseteq \mathcal{X}$, we infer that also $\min \left(\pi\left(\alpha^{\prime}\right)\right) \geq \beta>\alpha$, as sought.

\section{A generalization of $\mathbb{\bigotimes}^{-}(\kappa)$}

In this section and the next one, we shall present generalizations of the concepts that arose in Section 2. Here, we present a principle $\mathrm{P}_{\xi}^{-}(\kappa, \mu, \mathcal{R}, \theta, \mathcal{S}, \nu, \sigma)$ that generalizes the ladder system principle $\mathbb{\nabla}^{-}(\kappa)$. Then, in the next section, we shall present $\mathrm{P}_{\xi}^{\bullet}(\kappa, \mu, \mathcal{R}, \theta, \mathcal{S}, \nu)$, which serves as a generalization and weakening of the conjunction $\nabla^{-}(\kappa)+\diamond(\kappa)$.

4.1. Ladder systems. We assume the reader is comfortable with Conventions 1.2 from Page 5

Definition 4.1 ([BR19a $)$. Let $\mathcal{K}(\kappa):=\left\{x \in \mathcal{P}(\kappa) \mid x \neq \emptyset \& \operatorname{acc}^{+}(x) \subseteq x \&\right.$ $\sup (x) \notin x\}$ denote the collection of all nonempty $x \subseteq \kappa$ such that $x$ is a club subset of $\sup (x)$.

For each $C \in \mathcal{K}(\kappa)$, denote $\alpha_{C}:=\sup (C)$

For a binary relation $\mathcal{R}$ over $\mathcal{K}(\kappa)$, and a nonempty collection $\mathcal{S}$ of stationary subsets of $\kappa$, we shall define a principle $\mathrm{P}_{\xi}^{-}(\kappa, \mu, \mathcal{R}, \theta, \mathcal{S}, \nu, \sigma)$ in two stages. In the first stage, we focus on the first four parameters.

Definition 4.2. We say that $\left\langle\mathcal{C}_{\alpha} \mid \alpha<\kappa\right\rangle$ is a $\mathrm{P}_{\xi}^{-}(\kappa, \mu, \mathcal{R}, \ldots)$-sequence iff, for every $\alpha \in \operatorname{acc}(\kappa)$, all of the following hold:

- $\mathcal{C}_{\alpha} \subseteq\left\{C \in \mathcal{K}(\kappa) \mid \operatorname{otp}(C) \leq \xi \& \alpha_{C}=\alpha\right\}$;

- $0<\left|\mathcal{C}_{\alpha}\right|<\mu$;

- for all $C \in \mathcal{C}_{\alpha}$ and $\bar{\alpha} \in \operatorname{acc}(C)$, there exists $D \in \mathcal{C}_{\bar{\alpha}}$ with $D \mathcal{R} C$.

Convention 4.3. If we omit the subscript $\xi$, then we mean that $\xi:=\kappa$.

Convention 4.4. We shall always assume that $\mathcal{C}_{0}:=\{\emptyset\}$ and $\mathcal{C}_{\alpha+1}:=\{\{\alpha\}\}$ for all $\alpha<\kappa$. Likewise, whenever we construct a $\mathrm{P}_{\xi}^{-}(\kappa, \mu, \mathcal{R}, \ldots)$-sequence $\left\langle\mathcal{D}_{\alpha} \mid \alpha<\kappa\right\rangle$, we shall never bother to define $\mathcal{D}_{0}$ and $\mathcal{D}_{\alpha+1}$ for $\alpha<\kappa$.

Example 4.5. The binary relations over $\mathcal{K}(\kappa)$ that fit as the parameter $\mathcal{R}$ should be understood as coherence relations. The basic example is the end-extension relation, $\sqsubseteq$, where, for $C, D \in \mathcal{K}(\kappa)$, we define $C \sqsubseteq D$ iff $C=D \cap \alpha_{C}$. More nuanced binary relations over $\mathcal{K}(\kappa)$ are obtained by modifying the $\sqsubseteq$ relation as follows:

- We define $C \sqsubseteq^{*} D$ iff there exists $\gamma<\alpha_{C}$ such that $C \backslash \gamma \sqsubseteq D \backslash \gamma$;

- For $\mathcal{R} \in\left\{\sqsubseteq, \sqsubseteq^{*}\right\}$, we define $C \chi \mathcal{R} D$ iff $\left((C \mathcal{R} D)\right.$ or $\left.\left(\operatorname{cf}\left(\alpha_{C}\right)<\chi\right)\right)$;

- For $\mathcal{R} \in\left\{\sqsubseteq, \sqsubseteq^{*}\right\}$, we define $C \mathcal{R}_{\chi} D$ iff $((C \mathcal{R} D)$ or $(\operatorname{otp}(D)<\chi$ and $\operatorname{nacc}(D)$ consists only of successor ordinals $)$ );

- For any binary relation $\mathcal{R}$ over $\mathcal{K}(\kappa)$ and any class $\Omega \subseteq$ ORD, we define $C^{\Omega} \mathcal{R} D$ iff $\left((C \mathcal{R} D)\right.$ and $\left.\left(\alpha_{C} \notin \Omega\right)\right)$.

$\mathrm{P}^{-}(\kappa, 2, \sqsubseteq, \ldots)$ - and $\mathrm{P}_{\lambda}^{-}\left(\lambda^{+}, 2,{ }_{\lambda} \sqsubseteq, \ldots\right)$-sequences may be constructed in ZFC, but there are stronger variations. For instance, Jensen's axiom $\square_{\lambda}$ (resp. $\square_{\lambda}^{*}$ ) is equivalent to the existence of a $\mathrm{P}_{\lambda}^{-}\left(\lambda^{+}, 2, \sqsubseteq, \ldots\right)$-sequence (resp. $\mathrm{P}_{\lambda}^{-}\left(\lambda^{+}, \lambda^{+}, \sqsubseteq, \ldots\right)$ sequence). More examples in this spirit may be found in BR17a.

Convention 4.6. We may put " $\infty$ " in place of $\mu$ in Definition 4.2, in which case we mean that $\left|\mathcal{C}_{\alpha}\right| \leq|\alpha|$ for every nonzero $\alpha<\kappa$. 
Remark 4.7. The relation $\sqsubseteq$ coincides with $\stackrel{\Omega}{\chi} \sqsubseteq$ for $(\Omega, \chi):=(\emptyset, 0)$, as well as $(\Omega, \chi):=(\emptyset, \omega)$. Note that if $\kappa=\lambda^{+}$is a successor cardinal, then for every relation

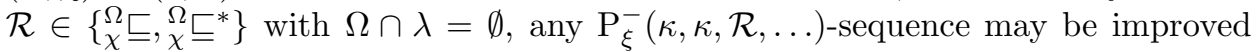
into a $\mathrm{P}_{\xi}^{-}(\kappa, \infty, \mathcal{R}, \ldots)$-sequence while preserving its further crucial features. The simple proof may be found in the construction before Claim 3.4.6 of [BR19b].

Let us stress that a study of coherence relations weaker than $\sqsubseteq$ is necessary. For instance, unlike coherent square sequences that are refuted by large cardinals, $\sqsubseteq \chi$-coherent square sequences provide an effective means to obtain optimal incompactness results above large cardinals (cf. [LR19]), as well as $\kappa$-Souslin trees in a model in which all $\kappa$-Aronszajn trees are nonspecial (cf. BR17a, Corollary 1.20, Examples 1.26 and 1.27]).

In BR19a, we introduced the following definition as a tool for manipulating and improving ladder systems.

Definition 4.8 ([BR19a, Definition 1.8]). A function $\Phi: \mathcal{K}(\kappa) \rightarrow \mathcal{K}(\kappa)$ is a postprocessing function iff for every $C \in \mathcal{K}(\kappa)$ :

- $\sup (\Phi(C))=\sup (C)$;

- $\operatorname{acc}(\Phi(C)) \subseteq \operatorname{acc}(C)$;

- $\Phi(C) \cap \bar{\alpha}=\Phi(C \cap \bar{\alpha})$ for every $\bar{\alpha} \in \operatorname{acc}(\Phi(C))$.

If, in addition, $\min (\Phi(C))=\min (C)(\operatorname{resp} . \operatorname{acc}(\Phi(C))=\operatorname{acc}(C))$ for every $C \in$ $\mathcal{K}(\kappa)$, then $\Phi$ is said to be min-preserving (resp. acc-preserving).

The point is that whenever $\left\langle\mathcal{C}_{\alpha} \mid \alpha<\kappa\right\rangle$ is a $\mathrm{P}_{\xi}^{-}\left(\kappa, \mu,{ }_{\chi} \sqsubseteq, \ldots\right)$-sequence, then for every postprocessing function $\Phi$, by setting $\mathcal{D}_{\alpha}:=\left\{\Phi(C) \mid C \in \mathcal{C}_{\alpha}\right\}$, we get that $\left\langle\mathcal{D}_{\alpha} \mid \alpha<\kappa\right\rangle$ is yet again a $\mathrm{P}_{\xi}^{-}\left(\kappa, \mu,{ }_{\chi}^{\Omega} \sqsubseteq, \ldots\right)$-sequence 14 We now present a sufficient condition for preserving all binary relations from Example 4.5.

Lemma 4.9. Suppose that $\mathfrak{x}=\left\langle x_{\gamma, \beta} \mid \gamma<\beta<\kappa\right\rangle$ is a triangular array of nonempty finite sets such that, for all $\gamma<\beta<\kappa, x_{\gamma, \beta} \subseteq(\gamma, \beta]$, and if $\beta$ is a successor ordinal, then $x_{\gamma, \beta}=\{\beta\}$. Define a corresponding function $\Phi_{\mathfrak{x}}: \mathcal{K}(\kappa) \rightarrow \mathcal{K}(\kappa)$ via:

$$
\Phi_{\mathfrak{x}}(C):=\{\min (C)\} \cup \bigcup\left\{x_{\gamma, \beta} \mid \gamma \in C, \beta=\min (C \backslash(\gamma+1))\right\} \cup \operatorname{acc}(C) .
$$

Then $\Phi_{\mathfrak{x}}$ is a min-preserving, acc-preserving postprocessing function. Furthermore, for every $\mathrm{P}_{\xi}^{-}(\kappa, \mu, \mathcal{R}, \ldots)$-sequence with $\mathcal{R}$ taken from Example 4.5, if we set $\mathcal{D}_{\alpha}:=\left\{\Phi_{\mathfrak{x}}(C) \mid C \in \mathcal{C}_{\alpha}\right\}$, then $\left\langle\mathcal{D}_{\alpha} \mid \alpha<\kappa\right\rangle$ is yet again a $\mathrm{P}_{\xi}^{-}(\kappa, \mu, \mathcal{R}, \ldots)$-sequence.

Proof. Left to the reader (cf. [BR19a, Lemma 2.8]).

4.2. Ladder systems with hitting features. We now arrive at the second stage of the definition of the proxy principle.

Definition 4.10 (Proxy principle). $\mathrm{P}_{\xi}^{-}(\kappa, \mu, \mathcal{R}, \theta, \mathcal{S}, \nu, \sigma)$ asserts the existence of a $\mathrm{P}_{\xi}^{-}(\kappa, \mu, \mathcal{R}, \ldots)$-sequence $\overrightarrow{\mathcal{C}}=\left\langle\mathcal{C}_{\alpha} \mid \alpha<\kappa\right\rangle$ satisfying the following hitting feature.

For every sequence $\left\langle B_{i} \mid i<\theta\right\rangle$ of cofinal subsets of $\kappa$, and every $S \in \mathcal{S}$, there exist stationarily many $\alpha \in S$ such that:

(1) $\left|\mathcal{C}_{\alpha}\right|<\nu$; and

(2) for all $C \in \mathcal{C}_{\alpha}$ and $i<\min \{\alpha, \theta\}$,

$$
\sup \left\{\gamma \in C \mid \operatorname{succ}_{\sigma}(C \backslash \gamma) \subseteq B_{i}\right\}=\alpha
$$

\footnotetext{
${ }^{14}$ In fact, postprocessing functions can be viewed as actions on square-like sequences, see Notation 2.15, Lemma 2.16 and Lemma 4.17 of BR19a]. Also, please keep Convention 4.4 in mind.
} 
Remark 4.11. The reader can verify that the proxy principle satisfies monotonicity properties with respect to most of its parameters: Any sequence witnessing $\mathrm{P}_{\xi}^{-}(\kappa, \mu, \mathcal{R}, \theta, \mathcal{S}, \nu, \sigma)$ remains a witness to the principle if any of $\xi, \mu$, or $\nu$ are increased; if $\theta$ or $\sigma$ are decreased; if $\mathcal{R}$ is weakened; if $\mathcal{S}$ is shrunk; or if any element of $\mathcal{S}$ is expanded. Furthermore, increasing $\chi$ or shrinking $\Omega$ both weaken the relations ${ }_{\chi}^{\Omega} \sqsubseteq,{ }_{\chi}^{\Omega} \sqsubseteq^{*},{ }^{\Omega} \sqsubseteq \chi$, and ${ }^{\Omega} \sqsubseteq_{\chi}^{*}$. Note also that $\mathrm{P}_{\xi}^{-}\left(\kappa, \mu,{ }^{\Omega} \mathcal{R}_{\chi}, \ldots\right)$ entails $\mathrm{P}_{\xi}^{-}\left(\kappa, \mu,{ }_{\chi}^{\Omega} \mathcal{R}, \ldots\right)$. These monotonicity properties will be used freely without explanation as the need arises.

Remark 4.12. In the special case $\sigma=1$, Equation (因) above is equivalent to the assertion that $\sup \left(\operatorname{nacc}(C) \cap B_{i}\right)=\alpha$. Thus, applying an argument just as in the proof of Corollary 3.4 , we infer that the principle $\otimes^{-}(\kappa)$ of Definition 2.16] is equivalent to the instance $\mathrm{P}^{-}(\kappa, 2, \sqsubseteq, 1,\{\kappa\}, 2,1)$.

Convention 4.13. In Definition 4.10, by putting " $<\theta$ " in place of $\theta$, we mean that $\overrightarrow{\mathcal{C}}$ simultaneously witnesses $\mathrm{P}_{\xi}^{-}\left(\kappa, \mu, \mathcal{R}, \theta^{\prime}, \mathcal{S}, \nu, \sigma\right)$ for all $\theta^{\prime}<\theta$.

Convention 4.14. In Definition 4.10, by putting " $<\sigma$ " in place of $\sigma$, we mean that $\overrightarrow{\mathcal{C}}$ simultaneously witnesses $\mathrm{P}_{\xi}^{-}\left(\kappa, \mu, \mathcal{R}, \theta, \mathcal{S}, \nu, \sigma^{\prime}\right)$ for all $\sigma^{\prime}<\sigma$.

We may also put " $<\infty$ " in place of $\sigma$, in which case we mean to replace the assertion of Equation (因) of Definition 4.10 by:

$$
\forall \sigma<\operatorname{otp}(C) \sup \left\{\gamma \in C \mid \operatorname{succ}_{\sigma}(C \backslash \gamma) \subseteq B_{i}\right\}=\alpha .
$$

Theorem 4.15. Suppose $\mathcal{R}$ is taken from Example 4.5. Then all of the following are equivalent:

(i) $\mathrm{P}_{\xi}^{-}(\kappa, \mu, \mathcal{R}, \theta, \mathcal{S}, \nu,<\omega)$;

(ii) $\mathrm{P}_{\xi}^{-}(\kappa, \mu, \mathcal{R}, \theta, \mathcal{S}, \nu, 2)$;

(iii) There exists a $\mathrm{P}_{\xi}^{-}(\kappa, \mu, \mathcal{R}, \ldots)$-sequence $\left\langle\mathcal{C}_{\alpha} \mid \alpha<\kappa\right\rangle$ satisfying the following. For every $S \in \mathcal{S}$ and every sequence $\left\langle B_{i} \mid i<\theta\right\rangle$ of cofinal subsets of $\kappa$, there exist stationarily many $\alpha \in S$ with $\left|\mathcal{C}_{\alpha}\right|<\nu$ such that, for all $C \in \mathcal{C}_{\alpha}$ and $i<\min \{\alpha, \theta\}$,

$$
\sup \left\{\delta \in B_{i} \cap \alpha \mid \min (C \backslash(\delta+1)) \in B_{i}\right\}=\alpha ;
$$

(iv) There exists a $\mathrm{P}_{\xi}^{-}(\kappa, \mu, \mathcal{R}, \ldots)$-sequence $\left\langle\mathcal{C}_{\alpha} \mid \alpha<\kappa\right\rangle$ satisfying the following. For every $S \in \mathcal{S}$, every sequence $\left\langle B_{i} \mid i<\theta\right\rangle$ of cofinal subsets of $\kappa$, and every every club $D \subseteq \kappa$, there exists $\alpha \in S \cap \operatorname{acc}(\kappa)$ with $\left|\mathcal{C}_{\alpha}\right|<\nu$ such that, for all $C \in \mathcal{C}_{\alpha}$ and $i<\min \{\alpha, \theta\}$,

$$
\sup \left\{\delta \in D \cap \alpha \mid \min (C \backslash(\delta+1)) \in B_{i}\right\}=\alpha ;
$$

(v) There exists a $\mathrm{P}_{\xi}^{-}(\kappa, \mu, \mathcal{R}, \ldots)$-sequence $\left\langle\mathcal{C}_{\alpha} \mid \alpha<\kappa\right\rangle$ satisfying the following. For every $S \in \mathcal{S}$ and every sequence $\left\langle\mathcal{B}_{i} \mid i<\theta\right\rangle$ with $\mathcal{B}_{i} \subseteq[\kappa]^{<\omega}$ and $\operatorname{mup}\left(\mathcal{B}_{i}\right)=\kappa$ for all $i<\theta 15$ there exist stationarily many $\alpha \in S$ with $\left|\mathcal{C}_{\alpha}\right|<\nu$ such that, for all $C \in \mathcal{C}_{\alpha}, i<\min \{\alpha, \theta\}$ and $\epsilon<\alpha$, there exist $\gamma, \beta$ with $\epsilon \leq \gamma<\beta<\alpha$ for which $C \cap(\gamma, \beta)$ is in $\mathcal{B}_{i}$;

(vi) There exists a $\mathrm{P}_{\xi}^{-}(\kappa, \mu, \mathcal{R}, \ldots)$-sequence $\left\langle\mathcal{C}_{\alpha} \mid \alpha<\kappa\right\rangle$ satisfying the following. For every $S \in \mathcal{S}$, every sequence $\left\langle B_{i} \mid i<\theta\right\rangle$ of cofinal subsets of $\kappa$, and every $n<\omega$, there exist stationarily many $\alpha \in S$ with $\left|\mathcal{C}_{\alpha}\right|<\nu$ such that, for all $C \in \mathcal{C}_{\alpha}$ and $i<\min \{\alpha, \theta\}$,

$$
\sup \left\{\gamma \in C \mid \operatorname{succ}_{n}(C \backslash \gamma)=\operatorname{succ}_{n}\left(B_{i} \backslash \gamma\right)\right\}=\alpha .
$$

\footnotetext{
${ }^{15}$ For the definition of mup, see Definition 3.8
} 
Proof. It is clear that any sequence witnessing (i) will witness (ii), any sequence witnessing (ii) will witness (iii), and any sequence witnessing (vi) will witness (i). Next, to see that any sequence witnessing (iii) will witness (iv), suppose that we are given a club $D$ and a sequence $\left\langle B_{i}\right| i\langle\theta\rangle$ of cofinal subsets of $\kappa$. For each $i<\theta$, by Lemma 3.3. fix a cofinal subset $B_{i}^{\prime}$ of $B_{i}$ that is $D$-separated. Then, for every $\alpha \in \operatorname{acc}(\kappa)$, every club $C$ in $\alpha$, and every $i<\theta$, if $\sup \left\{\delta \in B_{i}^{\prime} \cap \alpha \mid\right.$ $\left.\min (C \backslash(\delta+1)) \in B_{i}^{\prime}\right\}=\alpha$, then $\sup \left\{\delta \in D \cap \alpha \mid \min (C \backslash(\delta+1)) \in B_{i}\right\}=\alpha$.

(iv) $\Longrightarrow(\mathrm{v})$ : Fix an injection $\psi:[\kappa]^{<\omega} \rightarrow \operatorname{acc}(\kappa)$ such that, for all $x \in[\kappa]^{<\omega}$, $\psi(x)>\sup (x)$. Denote $\Omega:=\operatorname{Im}(\psi)$, and let $\pi: \Omega \rightarrow[\kappa]^{<\omega}$ denote the inverse of $\psi$.

Fix a triangular array $\mathfrak{x}=\left\langle x_{\gamma, \beta} \mid \gamma<\beta<\kappa\right\rangle$, such that for all $\gamma<\beta<\kappa$ :

- if $\beta \in \Omega$, then $x_{\gamma, \beta}=(\pi(\beta) \backslash(\gamma+1)) \cup\{\beta\}$;

- if $\beta \notin \Omega$, then $x_{\gamma, \beta}=\{\beta\}$.

Evidently, $x_{\gamma, \beta} \subseteq(\gamma, \beta]$ for all $\gamma<\beta<\kappa$. Thus, consider the corresponding postprocessing function $\Phi_{\mathfrak{x}}$ from Lemma 4.9. Let $\overrightarrow{\mathcal{C}}=\left\langle\mathcal{C}_{\alpha} \mid \alpha<\kappa\right\rangle$ be a sequence as in (iv). For every $\alpha \in \operatorname{acc}(\kappa)$, let $\mathcal{C}_{\alpha}^{\bullet}:=\left\{\Phi_{\mathfrak{x}}(C) \mid C \in \mathcal{C}_{\alpha}\right\}$, so that $\overrightarrow{\mathcal{C}}^{\bullet}:=\left\langle\mathcal{C}_{\alpha}^{\bullet} \mid \alpha<\kappa\right\rangle$ is a $\mathrm{P}_{\xi}^{-}(\kappa, \mu, \mathcal{R}, \ldots)$-sequence. The following claim shows that $\overrightarrow{\mathcal{C}^{\bullet}}$ witnesses $(\mathrm{v})$ :

Claim 4.15.1. Suppose $S \in \mathcal{S},\left\langle\mathcal{B}_{i} \mid i<\theta\right\rangle$ is a sequence with $\mathcal{B}_{i} \subseteq[\kappa]^{<\omega}$ and $\operatorname{mup}\left(\mathcal{B}_{i}\right)=\kappa$ for all $i<\theta$, and $D \subseteq \kappa$ is a club. Then there exists $\alpha \in S \cap D$ such that:

(1) $\left|\mathcal{C}_{\alpha}^{\bullet}\right|<\nu$; and

(2) for all $C^{\bullet} \in \mathcal{C}_{\alpha}^{\bullet}, i<\min \{\alpha, \theta\}$ and $\epsilon<\alpha$, there exist $\gamma, \beta$ with $\epsilon \leq \gamma<\beta<$ $\alpha$ for which $C^{\bullet} \cap(\gamma, \beta)$ is in $\mathcal{B}_{i}$.

Proof. For all $i<\theta$ and $\gamma<\kappa$, fix $b_{i}^{\gamma} \in \mathcal{B}_{i}$ with $\min \left(b_{i}^{\gamma}\right)>\gamma$. Fix a club $D^{\prime} \subseteq D$ such that, for all $\delta \in D^{\prime}$ :

- $\psi$ " $[\delta]^{<\omega} \subseteq \delta$;

- for all $\gamma<\delta$ and $i<\min \{\theta, \delta\}, b_{i}^{\gamma} \subseteq \delta$.

For each $i<\theta$, let

$$
B_{i}:=\left\{\psi\left(\{\gamma\} \cup b_{i}^{\gamma}\right) \mid \gamma \in D^{\prime}\right\} .
$$

Now, by the choice of $\overrightarrow{\mathcal{C}}$, fix $\alpha \in S \cap \operatorname{acc}(\kappa)$ such that:

- $\left|\mathcal{C}_{\alpha}\right|<\nu$, and

- for all $i<\min \{\alpha, \theta\}$ and $C \in \mathcal{C}_{\alpha}$,

$$
\sup \left\{\delta \in D^{\prime} \cap \alpha \mid \min (C \backslash(\delta+1)) \in B_{i}\right\}=\alpha .
$$

In particular, $\alpha \in \operatorname{acc}^{+}\left(D^{\prime}\right) \subseteq D^{\prime} \subseteq D$. Furthermore, $\left|\mathcal{C}_{\alpha}^{\bullet}\right| \leq\left|\mathcal{C}_{\alpha}\right|<\nu$. Finally, let $i<\min \{\alpha, \theta\}$ and $C^{\bullet} \in \mathcal{C}_{\alpha}^{\bullet}$ be arbitrary. Fix $C \in \mathcal{C}_{\alpha}$ such that $C^{\bullet}=\Phi_{\mathfrak{x}}(C)$. Let

$$
\Delta_{i}:=\left\{\delta \in D^{\prime} \cap \alpha \mid \min (C \backslash(\delta+1)) \in B_{i} \& \max \{i, \min (C)\}<\delta\right\} .
$$

By the choice of $\alpha, \Delta_{i}$ is cofinal in $\alpha$. Let $\delta \in \Delta_{i}$ be arbitrary. Set $\beta:=\min (C \backslash(\delta+$ $1))$ and $\beta^{-}:=\sup (C \cap \beta)$. As $\beta \in B_{i}$, let us fix $\gamma \in D^{\prime}$ such that $\pi(\beta)=\{\gamma\} \cup b_{i}^{\gamma}$. Evidently, $x_{\gamma, \beta}=b_{i}^{\gamma} \cup\{\beta\}$. As $\psi^{"}[\delta]^{<\omega} \subseteq \delta$ and $\beta>\delta$, we infer that $\pi(\beta) \notin[\delta]^{<\omega}$, so that $b_{i}^{\gamma} \nsubseteq \delta$. As $\delta \in D^{\prime}$ and $i<\min \{\theta, \delta\}$, this means that $\gamma \geq \delta$. So, $\min (\pi(\beta))=\gamma \geq \delta \geq \beta^{-}$, and hence $x_{\beta^{-}, \beta} \cap(\gamma, \beta)=b_{i}^{\gamma}$. It thus follows from the definition of $\Phi_{\mathfrak{x}}$ that $C^{\bullet} \cap(\gamma, \beta)=\left(x_{\beta^{-}, \beta} \cap(\gamma, \beta)\right) \in \mathcal{B}_{i}$, as sought.

Finally, to see that any sequence witnessing (v) will witness (vi), let $\left\langle B_{i} \mid i<\theta\right\rangle$ be an arbitrary sequence of cofinal subsets of $\kappa$, and let $n<\omega$ be arbitrary. For each $i<\theta$, set $\mathcal{B}_{i}:=\left\{\operatorname{succ}_{n+1}\left(B_{i} \backslash \epsilon\right) \mid \epsilon<\kappa\right\}$. Then, for every $\alpha \in \operatorname{acc}(\kappa)$, every club $C$ in $\alpha$, every $i<\theta$, and every $\gamma, \beta$ such that $C \cap(\gamma, \beta)$ is in $\mathcal{B}_{i}$, setting $\gamma^{\prime}:=\min (C \cap(\gamma, \beta))$, we obtain $\operatorname{succ}_{n}\left(C \backslash \gamma^{\prime}\right)=\operatorname{succ}_{n}\left(B_{i} \backslash \gamma^{\prime}\right)$. 
It is clear that a sequence witnessing any one of the clauses of Theorem 4.15 will also witness $\mathrm{P}_{\xi}^{-}(\kappa, \mu, \mathcal{R}, \theta, \mathcal{S}, \nu, 1)$. Clause (1) of the following Theorem shows that in the presence of $\boldsymbol{\beta}(\kappa)$, the principles are equivalent.

Theorem 4.16. Suppose $\mathbf{\beta}(\kappa)$ and $\mathrm{P}_{\xi}^{-}(\kappa, \mu, \mathcal{R}, \theta, \mathcal{S}, \nu, 1)$ both hold, with $\mathcal{R}$ taken from Example 4.5. Then:

(1) $\mathrm{P}_{\xi}^{-}(\kappa, \mu, \mathcal{R}, \theta, \mathcal{S}, \nu,<\omega)$ holds;

(2) If $0<\theta<\omega$, then $\mathrm{P}_{\xi}^{-}(\kappa, \mu, \mathcal{R},<\omega, \mathcal{S}, \nu,<\omega)$ holds.

Proof. (1) Denote $\Omega:=\operatorname{acc}(\kappa)$. By $\boldsymbol{\phi}(\kappa)$ and Lemma 3.9. we may fix a sequence $\overrightarrow{\mathcal{X}}=\left\langle\mathcal{X}_{\beta} \mid \beta \in \Omega\right\rangle$ such that:

(a) for every $\beta \in \Omega, \mathcal{X}_{\beta} \subseteq[\beta]^{<\omega}$ with $\operatorname{mup}\left(\mathcal{X}_{\beta}\right)=\beta$;

(b) for every $\mathcal{X} \subseteq[\kappa]^{<\omega}$ with $\operatorname{mup}(\mathcal{X})=\kappa$, there are stationarily many $\beta \in \Omega$ for which $\mathcal{X}_{\beta} \subseteq \mathcal{X}$.

Fix a triangular array $\mathfrak{x}=\left\langle x_{\gamma, \beta} \mid \gamma<\beta<\kappa\right\rangle$ such that for all $\gamma<\beta<\kappa$ :

- if $\beta \in \Omega$, then $x_{\gamma, \beta} \in \mathcal{X}_{\beta} \backslash\{\emptyset\}$ with $\min \left(x_{\gamma, \beta}\right)>\gamma$;

- if $\beta \notin \Omega$, then $x_{\gamma, \beta}=\{\beta\}$.

Now, consider the corresponding postprocessing function $\Phi_{\mathfrak{x}}$ from Lemma 4.9 . Let $\overrightarrow{\mathcal{C}}=\left\langle\mathcal{C}_{\alpha} \mid \alpha<\kappa\right\rangle$ be a sequence witnessing $\mathrm{P}_{\xi}^{-}(\kappa, \mu, \mathcal{R}, \theta, \mathcal{S}, \nu, 1)$. For every $\alpha \in \operatorname{acc}(\kappa)$, let $\mathcal{D}_{\alpha}:=\left\{\Phi_{\mathfrak{x}}(C) \mid C \in \mathcal{C}_{\alpha}\right\}$, so that $\overrightarrow{\mathcal{D}}:=\left\langle\mathcal{D}_{\alpha} \mid \alpha<\kappa\right\rangle$ is a $\mathrm{P}_{\xi}^{-}(\kappa, \mu, \mathcal{R}, \ldots)$-sequence. To see that $\mathrm{P}_{\xi}^{-}(\kappa, \mu, \mathcal{R}, \theta, \mathcal{S}, \nu,<\omega)$ holds, we shall verify that $\overrightarrow{\mathcal{D}}$ witnesses Clause (v) of Theorem 4.15 .

Claim 4.16.1. Suppose $S \in \mathcal{S}$ and $\left\langle\mathcal{B}_{i} \mid i<\theta\right\rangle$ is a sequence with $\mathcal{B}_{i} \subseteq[\kappa]^{<\omega}$ and $\operatorname{mup}\left(\mathcal{B}_{i}\right)=\kappa$ for all $i<\theta$. Then there exist stationarily many $\alpha \in S$ such that:

(1) $\left|\mathcal{D}_{\alpha}\right|<\nu$; and

(2) for all $D \in \mathcal{D}_{\alpha}, i<\min \{\alpha, \theta\}$ and $\epsilon<\alpha$, there exist $\gamma, \beta$ with $\epsilon \leq \gamma<\beta<$ $\alpha$ for which $D \cap(\gamma, \beta)$ is in $\mathcal{B}_{i}$.

Proof. For every $i<\theta$, we know from our choice of $\overrightarrow{\mathcal{X}}$ that $B_{i}:=\left\{\beta \in \Omega \mid \mathcal{X}_{\beta} \subseteq \mathcal{B}_{i}\right\}$ is stationary. Thus, by our choice of $\overrightarrow{\mathcal{C}}$, the following set is stationary:

$$
S^{\prime}:=\left\{\alpha \in S|| \mathcal{C}_{\alpha} \mid<\nu \& \forall i<\min \{\alpha, \theta\} \forall C \in \mathcal{C}_{\alpha}\left[\sup \left(\operatorname{nacc}(C) \cap B_{i}\right)=\alpha\right]\right\} .
$$

Now, let $\alpha \in S^{\prime}$ be arbitrary. We have $\left|\mathcal{D}_{\alpha}\right| \leq\left|\mathcal{C}_{\alpha}\right|<\nu$. Finally, let $i<\min \{\alpha, \theta\}$ and $D \in \mathcal{D}_{\alpha}$ be arbitrary. Pick $C \in \mathcal{C}_{\alpha}$ such that $D=\Phi_{\mathfrak{r}}(C)$. For all $\beta \in$ $\operatorname{nacc}(C) \cap B_{i}$ above $\min (C)$, we have $\mathcal{X}_{\beta} \subseteq \mathcal{B}_{i}$ so that for $\gamma:=\sup (C \cap \beta)$, we obtain $x_{\gamma, \beta} \in \mathcal{B}_{i}$ and $x_{\gamma, \beta} \subseteq(\gamma, \beta)$, and we infer from the definition of $\Phi_{\mathfrak{x}}$ that $D \cap(\gamma, \beta)=x_{\gamma, \beta} \in \mathcal{B}_{i}$.

Thus, $\mathrm{P}_{\xi}^{-}(\kappa, \mu, \mathcal{R}, \theta, \mathcal{S}, \nu,<\omega)$ follows, by the implication $(v) \Longrightarrow(i)$ of Theorem 4.15 .

(2) Left to the reader to show that a sequence as in Clause (v) of Theorem 4.15 with $\theta=1$ also witnesses $\mathrm{P}_{\xi}^{-}(\kappa, \mu, \mathcal{R},<\omega, \mathcal{S}, \nu,<\omega)$.

Remark 4.17. Clause (1) of the preceding is used in the proofs of Theorems 3.11 and 3.12 of [LR19, and also, implicitly, in the proof of Corollary 3.15(2) of the same paper.

In BR17a, p. 1953], we wrote that if we omit $\sigma$, then we mean that $\sigma=1$, but given Theorems 4.15 and 4.16, we now upgrade it as follows.

Convention 4.18. Whenever we omit $\sigma$, we mean that we put " $<\omega$ " in place of $\sigma$. 
4.3. Jensen's classical result and nonreflecting sets. In [Jen72, Theorem 6.2], Jensen showed that, assuming $V=L$, there is a $\kappa$-Souslin tree for every regular uncountable cardinal $\kappa$ that is not weakly compact. The proof of that theorem actually derives a $\kappa$-Souslin tree from the existence of a stationary set $E \subset \kappa$ for which $\square(E)+\diamond(E)$ holds 16 where $\square(E)$ is the principle asserting the existence of a $\mathrm{P}^{-}\left(\kappa, 2,{ }^{E} \sqsubseteq, \ldots\right)$-sequence, and should not be confused with the weaker principle $\square(\kappa)$. Here, we show how to redirect this result through our framework 17

Corollary 4.19. Suppose $\square(E)+\diamond(E)$ holds for some stationary $E \subset \kappa$. Then:

(1) $\mathrm{P}^{-}\left(\kappa, 2, \coprod^{*}, 1,\{E\}, 2\right)$;

(2) If $\kappa>\aleph_{1}$, then $\mathrm{P}^{-}\left(\kappa, 2, \sqsubseteq^{*}, 1,\{S\}, 2\right)$ holds for every stationary $S \subseteq \kappa$.

Proof. (1) By Corollary 4.22 below, there exists a stationary $\Omega \subseteq E$ such that $\mathrm{P}^{-}\left(\kappa, 2,{ }_{\chi} \sqsubseteq^{*}, 1,\{\Omega\}, 2\right)$ holds for $\chi:=\omega$. In particular, $\mathrm{P}^{-}\left(\kappa, 2, \sqsubseteq^{*}, 1,\{E\}, 2\right)$ holds.

(2) This follows from Clause (1), Rin17, Theorem 4.13], and Theorem 4.16.

We now present the main technical lemma of this subsection. In order to enable applications of this lemma to scenarios beyond the scope of this paper (see [RZ20]), we temporarily suspend our usual convention requiring $\mu \leq \kappa$. Thus, for the rest of this subsection only, a $\mathrm{P}^{-}\left(\kappa, \kappa^{+}, \mathcal{R}, \ldots\right)$-sequence is a sequence just as in Definition 4.2, with the second bullet replaced by $0<\left|\mathcal{C}_{\alpha}\right| \leq \kappa$.

Lemma 4.20. Suppose $\kappa^{\aleph_{0}}=\kappa, S \subseteq \kappa$ is stationary, $\boldsymbol{中}(S)$ holds, and $\overrightarrow{\mathcal{C}}^{0}=\left\langle\mathcal{C}_{\alpha}^{0}\right|$ $\alpha<\kappa\rangle$ is a $\mathrm{P}^{-}\left(\kappa, \kappa^{+},{ }_{\chi} \sqsubseteq^{*}, \ldots\right)$-sequence. Then there exist:

(1) a subset $\Omega \subseteq S \cap \operatorname{acc}(\kappa)$;

(2) a sequence $\left\langle X_{\beta} \mid \beta \in \Omega\right\rangle$, where each $X_{\beta}$ is a cofinal subset of $\beta$;

(3) $a \mathrm{P}^{-}\left(\kappa, \kappa^{+}, \Omega_{\chi} \coprod^{*}, \ldots\right)$-sequence, $\left\langle\mathcal{C}_{\alpha} \mid \alpha<\kappa\right\rangle$, with $\left|\mathcal{C}_{\alpha}\right| \leq\left|\mathcal{C}_{\alpha}^{0}\right|$ for all $\alpha<\kappa$, together satisfying the following hitting feature:

(4) for every cofinal $X \subseteq \kappa$, there exists some $\alpha \in \Omega$ such that $\mathcal{C}_{\alpha}$ is a singleton, say, $\mathcal{C}_{\alpha}=\left\{C_{\alpha}\right\}$, and

$$
\sup \left\{\beta \in \operatorname{nacc}\left(C_{\alpha}\right) \cap \Omega \mid X_{\beta} \subseteq X\right\}=\alpha .
$$

Proof. Following Convention 4.4, we assume that $\mathcal{C}_{\alpha+1}^{0}=\{\{\alpha\}\}$ for every $\alpha<\kappa$. Furthermore, by ${ }_{\chi}^{S} \coprod^{*}$-coherence of the sequence $\overrightarrow{\mathcal{C}}^{0}$, we may assume that $\left|\mathcal{C}_{\alpha}^{0}\right|=1$ for every $\alpha \in S$.

Let $\left\langle X_{\beta}^{n} \mid \beta \in S, n \leq \omega\right\rangle$ be given by Lemma 3.5 using $\theta:=\omega$. Without loss of generality, for all $\beta \in S \cap \operatorname{acc}(\kappa)$ and $n \leq \omega, X_{\beta}^{n}$ is a cofinal subset of $\beta$.

Suppose first that, for every cofinal $X \subseteq \kappa$, the set

$$
B_{X}:=\left\{\beta \in S \cap E_{\omega}^{\kappa} \mid X_{\beta}^{\omega} \subseteq X\right\}
$$

is stationary. In this case, let $\Omega:=S \cap E_{\omega}^{\kappa}$, define $\left\langle X_{\beta} \mid \beta \in \Omega\right\rangle$ via $X_{\beta}:=X_{\beta}^{\omega}$, and define a sequence $\overrightarrow{\mathcal{C}}=\left\langle\mathcal{C}_{\alpha} \mid \alpha<\kappa\right\rangle$ by letting $\mathcal{C}_{\alpha}:=\mathcal{C}_{\alpha}^{0}$ for every $\alpha \notin \Omega$, and $\mathcal{C}_{\alpha}:=\left\{X_{\alpha}^{\omega}\right\}$ for every $\alpha \in \Omega$. It is clear that $\overrightarrow{\mathcal{C}}$ is a $\mathrm{P}^{-}\left(\kappa, \kappa^{+},{ }_{\chi}^{S} \sqsubseteq^{*}, \ldots\right)$-sequence, satisfying $\left|\mathcal{C}_{\alpha}\right| \leq\left|\mathcal{C}_{\alpha}^{0}\right|$ for all $\alpha<\kappa$. In particular, it is a $\mathrm{P}^{-}\left(\kappa, \kappa^{+}, \chi_{\chi} \sqsubseteq^{*}, \ldots\right)-$ sequence. To see that Clause (4) is satisfied, let $X \subseteq \kappa$ be an arbitrary cofinal set. As $B_{X}$ is stationary, it is in particular cofinal, so that $B_{B_{X}}$ is stationary as well. Pick $\alpha \in B_{B_{X}}$. Then $\alpha \in \Omega, \mathcal{C}_{\alpha}=\left\{X_{\alpha}^{\omega}\right\}$, and $X_{\alpha}^{\omega} \subseteq B_{X}$. So, if $C_{\alpha}$ denotes the unique element of $\mathcal{C}_{\alpha}$, then every $\beta \in \operatorname{nacc}\left(C_{\alpha}\right)$ is an element of $B_{X}$, so that $\beta \in S \cap E_{\omega}^{\kappa}=\Omega$ and $X_{\beta}=X_{\beta}^{\omega} \subseteq X$, as sought.

\footnotetext{
${ }^{16}$ See [Dev84, Theorem IV.2.4] and [Sch14 Lemma 11.68].

${ }^{17}$ By Theorem 6.8 together with Proposition [5.10 the instances obtained in Corollary 4.19 suffice for the construction of a $\kappa$-Souslin tree
} 
From now on, suppose that there exists a cofinal $X^{\omega} \subseteq \kappa$ for which $B_{X^{\omega}}$ is nonstationary. Denote $\Omega^{0}:=S \cap E_{>\omega}^{\kappa}$. It follows that, for every sequence $\left\langle X^{n}\right|$ $n<\omega\rangle$ of cofinal subsets of $\kappa$, there exist stationarily many $\beta \in \Omega^{0}$, such that, for all $n<\omega, X_{\beta}^{n} \subseteq X^{n}$. In particular, $\kappa \geq \aleph_{2}$. The proof in this case is based on the arguments of [KS93, Theorem 3] and BR19c, Claim 3.8.1].

We have already defined $\Omega^{0}$ and $\overrightarrow{\mathcal{C}^{0}}$. We now attempt to construct a sequence $\left\langle\left(\Omega^{n+1}, \overrightarrow{\mathcal{C}^{n+1}}, X^{n}\right) \mid n<\omega\right\rangle$ satisfying the following properties for all $n<\omega$ :

(a) $\Omega^{n+1} \subseteq \Omega^{n}$

(b) $\overrightarrow{\mathcal{C}^{n}}=\left\langle\mathcal{C}_{\alpha}^{n} \mid \alpha<\kappa\right\rangle=\left\langle\left\{C_{\alpha, \iota}^{n} \mid \iota<\kappa\right\} \mid \alpha<\kappa\right\rangle$ is a $\mathrm{P}^{-}\left(\kappa, \kappa^{+}, \Omega^{\Omega^{n}} \sqsubseteq^{*}, \ldots\right)-$ sequence 18 such that $\mathcal{C}_{\alpha+1}^{n}=\{\{\alpha\}\}$ for every $\alpha<\kappa$, and $\left|\mathcal{C}_{\alpha}^{n}\right|=1$ for every $\alpha \in S$;

(c) $C_{\alpha, \iota}^{n} \subseteq C_{\alpha, \iota}^{n+1}$ for every $\alpha<\kappa$ and every $\iota<\kappa$;

(d) for every $\alpha<\kappa$ and all $\iota, \iota^{\prime}<\kappa$, if $C_{\alpha, \iota}^{n}=C_{\alpha, \iota^{\prime}}^{n}$, then $C_{\alpha, \iota}^{n+1}=C_{\alpha, \iota^{\prime}}^{n+1}$;

(e) $X^{n}$ is a cofinal subset of $\kappa$;

(f) $\sup \left(C_{\alpha, 0}^{n} \cap \Omega^{n+1}\right)<\alpha$ for every $\alpha \in \Omega^{n}$;

(g) for every $\alpha \in \operatorname{acc}(\kappa)$, all $\iota, \iota^{\prime}<\kappa$, every $\bar{\alpha} \in \operatorname{acc}\left(C_{\alpha, \iota^{\prime}}^{n}\right)$, and every $\eta \in$ $C_{\bar{\alpha}, \iota}^{n} \cap C_{\alpha, \iota^{\prime}}^{n}$, if $C_{\bar{\alpha}, \iota}^{n} \backslash \eta=C_{\alpha, \iota^{\prime}}^{n} \cap[\eta, \bar{\alpha})$ then $C_{\bar{\alpha}, \iota}^{n+1} \backslash \eta=C_{\alpha, \iota^{\prime}}^{n+1} \cap[\eta, \bar{\alpha})$;

(h) $\operatorname{nacc}\left(C_{\alpha, \iota}^{n}\right) \cap \Omega^{n+1} \subseteq \operatorname{nacc}\left(C_{\alpha, \iota}^{n+1}\right)$ for all $\alpha, \iota<\kappa$;

(i) for all $\alpha, \iota<\kappa$ and any two consecutive points $\beta^{-}<\beta$ of $C_{\alpha, \iota}^{n}$, either $C_{\alpha, \iota}^{n+1} \cap\left(\beta^{-}, \beta\right)=\emptyset$ or $C_{\alpha, \iota}^{n+1} \cap\left[\beta^{-}, \beta\right)=C_{\beta, 0}^{n+1} \backslash \beta^{-19}$

We proceed by recursion. Fix $n<\omega$ for which $\Omega^{n}$ and $\overrightarrow{\mathcal{C}^{n}}$ have already been successfully constructed. By (a) and (b) $\left\langle X_{\beta}^{n} \mid \beta \in \Omega^{n}\right\rangle$ and $\overrightarrow{\mathcal{C}^{n}}$ satisfy properties (1)-(3) of the conclusion of the lemma. If they satisfy property (4) as well, then the lemma is proven and we abandon the recursive construction at this point.

Otherwise, let us pick a cofinal subset $X^{n} \subseteq \kappa$ witnessing the failure of (4). That is, $\sup \left(\operatorname{nacc}\left(C_{\alpha, 0}^{n}\right) \cap \Omega^{n+1}\right)<\alpha$ for every $\alpha \in \Omega^{n}$, where we define:

$$
\Omega^{n+1}:=\left\{\beta \in \Omega^{n} \mid X_{\beta}^{n} \subseteq X^{n}\right\} .
$$

Define $\overrightarrow{\mathcal{C}}^{n+1}=\left\langle\mathcal{C}_{\alpha}^{n+1} \mid \alpha<\kappa\right\rangle$ by recursion over $\alpha<\kappa$, as follows. Let $\mathcal{C}_{0}^{n+1}:=$ $\{\emptyset\}$, and $\mathcal{C}_{\alpha+1}^{n+1}:=\{\{\alpha\}\}$ for every $\alpha<\kappa$. Now, if $\alpha \in \operatorname{acc}(\kappa)$ and $\left\langle\mathcal{C}_{\bar{\alpha}}^{n+1} \mid \bar{\alpha}<\alpha\right\rangle$ has already been defined, then, for every $\iota<\kappa$, let:

$$
C_{\alpha, \iota}^{n+1}:=C_{\alpha, \iota}^{n} \cup \bigcup\left\{C_{\beta, 0}^{n+1} \backslash \sup \left(C_{\alpha, \iota}^{n} \cap \beta\right) \mid \beta \in \operatorname{nacc}\left(C_{\alpha, \iota}^{n}\right) \backslash \Omega^{n+1}\right\} .
$$

Claim 4.20.1. $\Omega^{n+1}, \overrightarrow{\mathcal{C}}^{n+1}$, and $X^{n}$ satisfy properties $(a)(i)$ of the recursion.

Proof. (a) (c) (d), (e), (g), (h), and (i) are easily verified from the construction.

(f) Consider any given $\alpha \in \Omega^{n}$. By ${ }_{\chi}^{\Omega^{n}} \sqsubseteq{ }^{*}$-coherence of $\overrightarrow{\mathcal{C}^{n}}$, we obtain $\operatorname{acc}\left(C_{\alpha, 0}^{n}\right) \cap$ $\Omega^{n}=\emptyset$, so that certainly $\operatorname{acc}\left(C_{\alpha, 0}^{n}\right) \cap \Omega^{n+1}=\emptyset$. Thus $C_{\alpha, 0}^{n} \cap \Omega^{n+1}=\operatorname{nacc}\left(C_{\alpha, 0}^{n}\right) \cap$ $\Omega^{n+1}$, and it follows from our choice of $X^{n}$ and $D^{n}$ that

$$
\sup \left(C_{\alpha, 0}^{n} \cap \Omega^{n+1}\right)=\sup \left(\operatorname{nacc}\left(C_{\alpha, 0}^{n}\right) \cap \Omega^{n+1}\right)<\alpha .
$$

(b) It is clear from the construction that $C_{\alpha, \iota}^{n+1}$ is a club subset of $\alpha$ for every limit ordinal $\alpha<\kappa$, that $\mathcal{C}_{\alpha+1}^{n+1}=\{\{\alpha\}\}$ for every $\alpha<\kappa$, and (using (d) that $0<\left|\mathcal{C}_{\alpha}^{n+1}\right| \leq\left|\mathcal{C}_{\alpha}^{n}\right| \leq\left|\mathcal{C}_{\alpha}^{0}\right|$ for every $\alpha<\kappa$ and $\left|\mathcal{C}_{\alpha}^{n+1}\right|=1$ for every $\alpha \in S$.

To verify ${ }_{\chi}^{\Omega^{n+1}} \sqsubseteq^{*}$-coherence of the sequence $\overrightarrow{\mathcal{C}}^{n+1}$, consider any given $\alpha \in \operatorname{acc}(\kappa)$, $\iota<\kappa$, and $\bar{\alpha} \in \operatorname{acc}\left(C_{\alpha, \iota}^{n+1}\right)$; we shall show that $\bar{\alpha} \notin \Omega^{n+1}$, and also find some $\iota^{\prime}<\kappa$ such that $C_{\bar{\alpha}, \iota^{\prime}}^{n+1} \chi \sqsubseteq^{*} C_{\alpha, \iota}^{n+1}$, by considering three cases. We may assume, as an

\footnotetext{
${ }^{18}$ The enumeration $\left\{C_{\alpha, \iota}^{n} \mid \iota<\kappa\right\}$ of $\mathcal{C}_{\alpha}^{n}$ need not be injective.

${ }^{19} \mathrm{We}$ do not assume here that $\beta$ is a limit ordinal.
} 
induction hypothesis, that the restriction of $\overrightarrow{\mathcal{C}}^{n+1}$ up to $\alpha$, that is, $\left\langle\mathcal{C}_{\beta}^{n+1} \mid \beta<\alpha\right\rangle$, is already known to be ${\underset{\chi}{n+1}}^{n+}$-coherent.

- Suppose $\bar{\alpha} \in \operatorname{acc}\left(C_{\alpha, \iota}^{n}\right)$. Then by $\stackrel{\Omega}{\chi}^{n} \sqsubseteq^{*}$-coherence of $\overrightarrow{\mathcal{C}^{n}}$, it follows that $\bar{\alpha} \notin \Omega^{n}$ and also we can choose some $\iota^{\prime}<\kappa$ such that $C_{\bar{\alpha}, \iota^{\prime}}^{n} \chi \sqsubseteq^{*} C_{\alpha, \iota}^{n}$. As $\Omega^{n+1} \subseteq \Omega^{n}$, we obtain $\bar{\alpha} \notin \Omega^{n+1}$. If $\operatorname{cf}(\bar{\alpha})<\chi$, then automatically $C_{\bar{\alpha}, 0}^{n+1} \quad \sqsubseteq^{*} C_{\alpha, \iota}^{n+1}$. Otherwise, we have $C_{\bar{\alpha}, \iota^{\prime}}^{n} \sqsubseteq^{*} C_{\alpha, \iota}^{n}$, and we fix $\eta \in C_{\bar{\alpha}, \iota^{\prime}}^{n} \cap$ $C_{\alpha, \iota}^{n}$ such that $C_{\bar{\alpha}, \iota^{\prime}}^{n} \backslash \eta=C_{\alpha, \iota}^{n} \cap[\eta, \bar{\alpha})$. Then, by Clause (g), we obtain $C_{\bar{\alpha}, \iota^{\prime}}^{n+1} \backslash \eta=C_{\alpha, \iota}^{n+1} \cap[\eta, \bar{\alpha})$, so that $C_{\bar{\alpha}, \iota^{\prime}}^{n+1} \sqsubseteq^{*} C_{\alpha, \iota}^{n+1}$.

- Suppose $\bar{\alpha} \in \operatorname{nacc}\left(C_{\alpha, L}^{n}\right)$. As we have assumed that $\bar{\alpha} \in \operatorname{acc}\left(C_{\alpha, L}^{n+1}\right)$, we obtain from Clause $(\mathrm{h})$ that $\bar{\alpha} \notin \Omega^{n+1}$. Furthermore, it follows from Clause (i) (with $\beta:=\bar{\alpha}$ and $\beta^{-}:=\sup \left(C_{\alpha, \iota}^{n} \cap \beta\right)$ ) that $C_{\bar{\alpha}, 0}^{n+1} \sqsubseteq^{*} C_{\alpha, L}^{n+1}$.

- The remaining case is $\bar{\alpha} \notin C_{\alpha, \iota}^{n}$. Let $\beta^{-}<\beta$ be the two consecutive points of $C_{\alpha, \iota}^{n}$ such that $\beta^{-}<\bar{\alpha}<\beta$. In particular, $\beta \in \operatorname{nacc}\left(C_{\alpha, \iota}^{n}\right)$. Since $\bar{\alpha} \in \operatorname{acc}\left(C_{\alpha, L}^{n+1}\right) \cap\left(\beta^{-}, \beta\right)$, it follows from Clause (i) that $C_{\beta, 0}^{n+1} \backslash \beta^{-}=$ $C_{\alpha, L}^{n+1} \cap\left[\beta^{-}, \beta\right)$, so that $\bar{\alpha} \in \operatorname{acc}\left(C_{\beta, 0}^{n+1} \backslash \beta^{-}\right)$. As $\beta<\alpha$ and $\bar{\alpha} \in \operatorname{acc}\left(C_{\beta, 0}^{n+1}\right)$, by induction hypothesis we can choose some $\iota^{\prime}<\kappa$ such that $C_{\bar{\alpha}, \iota^{\prime}}^{n+1} \chi^{\Omega^{n+1}} \sqsubseteq^{*}$ $C_{\beta, 0}^{n+1}$. Thus, in particular, $\bar{\alpha} \notin \Omega^{n+1}$ and $C_{\bar{\alpha}, \iota^{\prime}}^{n+1} \chi \sqsubseteq^{*} C_{\beta, 0}^{n+1}$. If $\operatorname{cf}(\bar{\alpha})<\chi$, then automatically $C_{\bar{\alpha}, 0}^{n+1} \chi \sqsubseteq^{*} C_{\alpha, \iota}^{n+1}$. Otherwise, we have $C_{\bar{\alpha}, \iota^{\prime}}^{n+1} \sqsubseteq^{*} C_{\beta, 0}^{n+1}$, and as $\beta^{-}<\bar{\alpha}$, it follows that $C_{\bar{\alpha}, \iota^{\prime}}^{n+1} \sqsubseteq^{*} C_{\alpha, \iota}^{n+1}$, as sought.

If we reach the end of the above recursive process, then we are altogether equipped with a sequence $\left\langle\left(\overrightarrow{\mathcal{C}^{n}}, \Omega^{n}, X^{n}\right) \mid n<\omega\right\rangle$, from which we shall derive a contradiction. By the choice of $X^{\omega}$, the following set must be stationary:

$$
\Omega^{\bullet}:=\bigcap_{n<\omega} \Omega^{n}=\left\{\beta \in \Omega^{0} \mid \forall n<\omega\left(X_{\beta}^{n} \subseteq X^{n}\right)\right\} .
$$

Thus, $\operatorname{acc}^{+}\left(\Omega^{\bullet}\right)$ is a club in $\kappa$, and we may pick $\alpha \in \Omega^{\bullet} \cap \operatorname{acc}^{+}\left(\Omega^{\bullet}\right)$. For every $n<\omega$, put $\alpha_{n}:=\sup \left(C_{\alpha, 0}^{n} \cap \Omega^{n+1}\right)$, which by Clause (f) is $<\alpha$. As $\alpha \in \Omega^{\bullet} \subseteq \Omega^{0} \subseteq E_{>\omega}^{\kappa}$, we infer that $\alpha^{*}:=\sup _{n<\omega} \alpha_{n}$ is smaller than $\alpha$. Clearly,

$$
C_{\alpha, 0}^{n} \cap \Omega^{n+1} \cap\left(\alpha^{*}, \alpha\right)=\emptyset \text { for every } n<\omega .
$$

Since $\Omega^{\bullet} \cap \alpha$ is cofinal in $\alpha$, let us pick $\beta \in \Omega^{\bullet} \cap\left(\alpha^{*}, \alpha\right)$.

For all $n<\omega$, let $\beta_{n}:=\min \left(C_{\alpha, 0}^{n} \backslash \beta\right)$. As $\left\{C_{\alpha, 0}^{n} \mid n<\omega\right\}$ is a $\subseteq$-increasing chain, it follows that $\left\langle\beta_{n} \mid n<\omega\right\rangle$ is a $\leq$-decreasing sequence of ordinals, and hence stabilizes. Fix $n<\omega$ such that $\beta_{n}=\beta_{n+1}$. Since $\beta \in \Omega^{\bullet} \subseteq \Omega^{n+1},(* *)$ gives $\beta \notin C_{\alpha, 0}^{n}$, so that $\beta_{n}>\beta$. In particular, $\beta_{n}=\min \left(C_{\alpha, 0}^{n} \backslash(\beta+1)\right)$, so that $\beta_{n} \in \operatorname{nacc}\left(C_{\alpha, 0}^{n}\right)$. By $(* *)$ again and $\alpha>\beta_{n}>\beta>\alpha^{*}$, it follows that $\beta_{n} \in \operatorname{nacc}\left(C_{\alpha, 0}^{n}\right) \backslash \Omega^{n+1}$. Thus we infer from $(*)$ that

$$
C_{\alpha, 0}^{n+1} \cap\left[\sup \left(C_{\alpha, 0}^{n} \cap \beta_{n}\right), \beta_{n}\right)=C_{\beta_{n}, 0}^{n+1} \cap\left[\sup \left(C_{\alpha, 0}^{n} \cap \beta_{n}\right), \beta_{n}\right),
$$

and from $\beta_{n}=\min \left(C_{\alpha, 0}^{n} \backslash \beta\right)$, we obtain $\sup \left(C_{\alpha, 0}^{n} \cap \beta_{n}\right) \leq \beta$, and hence

$$
C_{\alpha, 0}^{n+1} \cap\left[\beta, \beta_{n}\right)=C_{\beta_{n}, 0}^{n+1} \cap\left[\beta, \beta_{n}\right) .
$$

Since $\beta<\beta_{n}$, it follows that $\beta_{n+1}=\min \left(C_{\alpha, 0}^{n+1} \backslash \beta\right)=\min \left(C_{\beta_{n}, 0}^{n+1} \backslash \beta\right)<\beta_{n}$, contradicting the choice of $n$ and completing the proof.

Remark 4.21. It follows from Lemma 3.3 that, in Clause (4) of the preceding, the existence of an $\alpha$ is equivalent to the existence of stationarily many such $\alpha$.

Corollary 4.22. Suppose $\kappa^{\aleph_{0}}=\kappa$, and $\mathbf{\$}(E)$ holds for a stationary $E \subseteq \kappa$. If there exists a $\mathrm{P}^{-}\left(\kappa, \mu,{ }_{\chi}^{E} \sqsubseteq^{*}, \ldots\right)$-sequence, then there exists a stationary $\Omega \subseteq E$ such that $\mathrm{P}^{-}\left(\kappa, \mu,{ }_{\chi} \sqsubseteq^{*}, 1,\{\Omega\}, 2\right)$ holds. 
Proof. Let $\Omega \subseteq E \cap \operatorname{acc}(\kappa),\left\langle X_{\beta} \mid \beta \in \Omega\right\rangle$, and $\left\langle\mathcal{C}_{\alpha} \mid \alpha<\kappa\right\rangle$ be given by Lemma4.20, using $S:=E$. For every $\beta \in \Omega$, let $\mathcal{X}_{\beta}:=\left\{\{\delta\} \mid \delta \in X_{\beta}\right\}$.

Set $\nu:=2$. By Clause (4) of Lemma 4.20 together with Remark 4.21, for every cofinal $X \subseteq \kappa$, letting $\mathcal{X}:=\{\{\delta\} \mid \delta \in X\}$ and $B_{0}:=\left\{\beta \in \Omega \mid \mathcal{X}_{\beta} \subseteq \mathcal{X}\right\}$, the set $S^{\prime}$ of all $\alpha \in \Omega$ such that:

- $\left|\mathcal{C}_{\alpha}\right|<\nu$, and

- for every $C \in \mathcal{C}_{\alpha}, \sup \left(\operatorname{nacc}(C) \cap B_{0}\right)=\alpha$

is stationary. The rest of the proof is now identical to that of Theorem 4.16(1), using $\mathcal{R}:={ }_{\chi}^{\Omega} \sqsubseteq *$ and $\theta:=1$.

Suppose $\kappa>\aleph_{1}$. Recall that a subset $\Omega \subseteq \kappa$ is said to be nonreflecting iff $\operatorname{Tr}(\Omega):=\{\alpha<\kappa \mid \operatorname{cf}(\alpha)>\omega \& \Omega \cap \alpha$ is stationary in $\alpha\}$ is empty. In particular, any $\Omega \subseteq \kappa$ for which there exists a $\mathrm{P}^{-}\left(\kappa, \mu,{ }^{\Omega} \mathcal{R}, \ldots\right)$-sequence (regardless of the value of $\mu$ ) must be a nonreflecting set, so that a set $S$ as in the hypothesis of Lemma 4.20 must be a nonreflecting stationary set. In BR19c, more applications of the existence of nonreflecting stationary sets were presented:

Fact $4.23([\overline{B R} 19 \mathrm{c}$, Theorem $\mathrm{A}])$. Suppose that $\mathrm{CH}_{\lambda}$ holds for a regular uncountable cardinal $\lambda=2^{<\lambda}$, and there exists a nonreflecting stationary subset of $E_{\neq \lambda^{+}}^{\lambda^{+}}$. Then $\mathrm{P}_{\lambda}^{-}\left(\lambda^{+}, \lambda^{+}, \sqsubseteq,<\lambda,\left\{\lambda^{+}\right\}, 2,<\lambda\right)$ and $\mathrm{P}^{-}\left(\lambda^{+}, \lambda^{+}, \sqsubseteq^{*}, 1,\left\{E_{\lambda}^{\lambda^{+}}\right\}, 2,1\right)$ both hold.

Fact 4.24 ([BR19c, Theorem B]). Suppose that $\square_{\lambda}^{*}+\mathrm{CH}_{\lambda}$ holds for a singular cardinal $\lambda=2^{<\lambda}$, and either of the following two conditions holds:

(1) there exists a nonreflecting stationary subset of $E_{\neq \mathrm{cf}(\lambda)}^{\lambda^{+}}$; or

(2) there exists a regressive function $f: E_{\mathrm{cf}(\lambda)}^{\lambda^{+}} \rightarrow \lambda^{+}$such that $f^{-1}\{i\}$ is nonreflecting for each $i<\lambda^{+}$.

Then $\mathrm{P}_{\lambda^{2}}^{-}\left(\lambda^{+}, \lambda^{+}, \sqsubseteq, \lambda^{+},\left\{\lambda^{+}\right\}, 2,<\lambda\right)$ holds.

Our next task is to prove analogous results for strongly inaccessible cardinals. For this, let us first recall a result from BR19a.

Fact 4.25 (Special case of [BR19a, Lemma 4.9]). Suppose that:

(a) $\diamond(\kappa)$ holds;

(b) $\overrightarrow{\mathcal{C}}=\left\langle\mathcal{C}_{\alpha} \mid \alpha<\kappa\right\rangle$ is a $\mathrm{P}^{-}\left(\kappa, \kappa,{ }^{\Omega} \sqsubseteq, \ldots\right)$-sequence for some fixed subset $\Omega \subseteq \kappa \backslash\{\omega\}$

(c) $\vec{C}=\left\langle C_{\alpha} \mid \alpha \in \operatorname{acc}(\kappa)\right\rangle$ is an element of $\prod_{\alpha \in \operatorname{acc}(\kappa)} \mathcal{C}_{\alpha}$;

(d) for every cofinal $B \subseteq \kappa$ and every $\Lambda^{\prime}<\kappa$, the following is stationary in $\kappa$ : $\left\{\alpha \in \kappa \backslash \Omega \mid \exists C \in \mathcal{C}_{\alpha}\left[\min (C)=\min (B), \Lambda^{\prime} \leq \operatorname{otp}(C), \operatorname{nacc}(C) \subseteq B\right]\right\}$.

Then there exists a $\mathrm{P}^{-}\left(\kappa, \kappa,{ }^{\Omega} \sqsubseteq, \ldots\right)$-sequence, $\overrightarrow{\mathcal{D}}=\left\langle\mathcal{D}_{\alpha} \mid \alpha<\kappa\right\rangle$, with an element $\vec{D}=\left\langle D_{\alpha} \mid \alpha \in \operatorname{acc}(\kappa)\right\rangle$ of $\prod_{\alpha \in \operatorname{acc}(\kappa)} \mathcal{D}_{\alpha}$, satisfying the following properties:

(1) $\left|\mathcal{D}_{\alpha}\right| \leq\left|\mathcal{C}_{\alpha}\right|$ for all $\alpha<\kappa$;

(2) For every cofinal $A \subseteq \kappa$, there exists a stationary $B \subseteq \kappa$ for which the set

$$
\left\{\alpha \in \operatorname{acc}(\kappa) \mid \operatorname{otp}\left(D_{\alpha}\right)=\alpha, \operatorname{nacc}\left(D_{\alpha}\right) \subseteq A\right\}
$$

covers the set

$\left\{\alpha \in \operatorname{acc}(\kappa) \mid \min \left(C_{\alpha}\right)=\min (B), \operatorname{otp}\left(C_{\alpha}\right)=\operatorname{cf}(\alpha), \operatorname{nacc}\left(C_{\alpha}\right) \subseteq B\right\}$.

Clause (2) of the next theorem is used in the proof of [BR19c, Corollary 4.13].

Theorem 4.26. Suppose that $S$ is a nonreflecting stationary subset of a strongly inaccessible cardinal $\kappa$ and $\diamond(S)$ holds. Then:

(1) $\mathrm{P}^{-}\left(\kappa, \kappa,{ }^{S} \sqsubseteq, 1,\{S\}, 2, \kappa\right)$ holds; 
(2) $\mathrm{P}^{-}\left(\kappa, \kappa,{ }^{S} \sqsubseteq, \kappa,\{S\}, 2,<\infty\right)$ holds.

Proof. Fix a $\diamond(S)$-sequence $\vec{A}=\left\langle A_{\alpha} \mid \alpha \in S\right\rangle$. As $S$ is nonreflecting, for every $\alpha \in \operatorname{acc}(\kappa)$, we may pick a closed and cofinal subset $B_{\alpha}$ of $\alpha$ with $\operatorname{otp}\left(B_{\alpha}\right)=\operatorname{cf}(\alpha)$ and $\operatorname{acc}\left(B_{\alpha}\right) \cap S=\emptyset$. Now, define a sequence $\vec{C}=\left\langle C_{\alpha} \mid \alpha \in \operatorname{acc}(\kappa)\right\rangle$, as follows.

- If $\alpha \in S$ and $A_{\alpha}$ is a cofinal subset of $\alpha$, then by Lemma 3.3. fix a cofinal subset $A_{\alpha}^{\prime}$ of $A_{\alpha}$ that is $B_{\alpha}$-separated, and then let $C_{\alpha}$ be the closure of $\left(A_{\alpha}^{\prime} \cup\left\{\min \left(A_{\alpha}\right)\right\}\right)$ in $\alpha$;

- Otherwise, let $C_{\alpha}:=B_{\alpha}$.

Note that, for every $\alpha \in \operatorname{acc}(\kappa), \operatorname{acc}\left(C_{\alpha}\right) \subseteq \operatorname{acc}\left(B_{\alpha}\right)$, so that $\operatorname{otp}\left(C_{\alpha}\right)=\operatorname{cf}(\alpha)$ and $\operatorname{acc}\left(C_{\alpha}\right) \cap S=\emptyset$.

Next, recalling Convention 4.4, we define a sequence $\overrightarrow{\mathcal{C}}=\left\langle\mathcal{C}_{\alpha} \mid \alpha<\kappa\right\rangle$, as follows. For $\alpha \in S$, we let $\mathcal{C}_{\alpha}:=\left\{C_{\alpha}\right\}$, and for $\alpha \in \operatorname{acc}(\kappa) \backslash S$, we let $\mathcal{C}_{\alpha}$ consist of all closed and cofinal subsets $C$ of $\alpha$ such that $\operatorname{acc}(C) \cap S=\emptyset$.

Evidently, $\vec{C}$ is an element of $\prod_{\alpha \in \operatorname{acc}(\kappa)} \mathcal{C}_{\alpha}$. In particular, and as $\kappa$ is strongly inaccessible, it follows that $\overrightarrow{\mathcal{C}}$ is a $\mathrm{P}^{-}\left(\kappa, \kappa,{ }^{\Omega} \sqsubseteq, \ldots\right)$-sequence for $\Omega:=S \backslash\{\omega\}$.

Claim 4.26.1. For every cofinal $B \subseteq \kappa$ and $\Lambda^{\prime}<\kappa$, the following sets are stationary in $\kappa$ :

(i) $\left\{\alpha \in \Omega \mid \min \left(C_{\alpha}\right)=\min (B), \operatorname{otp}\left(C_{\alpha}\right)=\operatorname{cf}(\alpha), \operatorname{nacc}\left(C_{\alpha}\right) \subseteq B\right\}$, and

(ii) $\left\{\alpha \in \kappa \backslash \Omega \mid \exists C \in \mathcal{C}_{\alpha}\left[\min (C)=\min (B), \Lambda^{\prime} \leq \operatorname{otp}(C), \operatorname{nacc}(C) \subseteq B\right]\right\}$.

Proof. Let $B$ be an arbitrary cofinal set.

(i) Consider the club $D:=\operatorname{acc}^{+}(B \backslash \omega)$. By the choice of $\vec{A}, S^{\prime}:=\{\alpha \in S \cap D \mid$ $\left.B \cap \alpha=A_{\alpha}\right\}$ is a stationary subset of $\Omega$. For every $\alpha \in S^{\prime}$, the definition of $C_{\alpha}$ implies that $\operatorname{otp}\left(C_{\alpha}\right)=\operatorname{cf}(\alpha), \operatorname{nacc}\left(C_{\alpha}\right) \subseteq A_{\alpha} \subseteq B$ and $\min \left(C_{\alpha}\right)=\min \left(A_{\alpha}\right)=$ $\min (B)$.

(ii) Let $\Lambda^{\prime}<\kappa$ be arbitrary. Let $D$ be an arbitrary club. By shrinking $D$, we may assume that $D \subseteq \operatorname{acc}^{+}(B)$. Set $\theta:=\max \left\{\Lambda^{\prime}, \aleph_{0}\right\}$. As $\kappa$ is a limit cardinal, we may fix $\delta \in \operatorname{acc}(D)$ with $\operatorname{cf}(\delta)=\theta^{++}$. Pick $\alpha \in \operatorname{acc}\left(C_{\delta}\right) \cap D$ with $\operatorname{cf}(\alpha)=\theta^{+}$. As $\vec{C}$ is a $\mathrm{P}^{-}\left(\kappa, \kappa,{ }^{\Omega} \sqsubseteq, \ldots\right)$-sequence, $\alpha \notin \Omega$. Finally, since $\alpha \in D \subseteq \operatorname{acc}^{+}(B)$, we infer that $B \cap \alpha$ is cofinal in $\alpha$. Let $A^{\prime}$ be a cofinal subset of $A:=B \cap \alpha$ that is $B_{\alpha}$-separated. Let $C$ be the closure of $\left(A^{\prime} \cup\{\min (A)\}\right)$ in $\alpha$. Then $C \in \mathcal{C}_{\alpha}$, $\min (C)=\min (A)=\min (B), \Lambda^{\prime}<\theta^{+}=\operatorname{otp}(C)$ and $\operatorname{nacc}(C) \subseteq A \subseteq B$.

By Clause (i) of the preceding claim, $\overrightarrow{\mathcal{C}}$ witnesses $\mathrm{P}^{-}\left(\kappa, \kappa,{ }^{\Omega} \sqsubseteq, 1,\{\Omega\}, 2, \kappa\right)$. As $S \triangle \Omega$ is finite, it follows that $\mathrm{P}^{-}\left(\kappa, \kappa,{ }^{S} \sqsubseteq, 1,\{S\}, 2, \kappa\right)$ holds, as well.

Next, by appealing to Fact 4.25 with $\overrightarrow{\mathcal{C}}$ and $\vec{C}$, we obtain a $\mathrm{P}^{-}\left(\kappa, \kappa,{ }^{\Omega} \sqsubseteq, \ldots\right)$ sequence $\overrightarrow{\mathcal{D}}=\left\langle\mathcal{D}_{\alpha} \mid \alpha<\kappa\right\rangle$, and an element $\vec{D}=\left\langle D_{\alpha} \mid \alpha \in \operatorname{acc}(\kappa)\right\rangle$ of $\prod_{\alpha \in \operatorname{acc}(\kappa)} \mathcal{D}_{\alpha}$, satisfying the following properties:

(1) $\left|\mathcal{D}_{\alpha}\right| \leq\left|\mathcal{C}_{\alpha}\right|$ for all $\alpha<\kappa$;

(2) For every cofinal $G \subseteq \kappa$, the following set is stationary:

$$
\left\{\alpha \in \Omega \mid \operatorname{otp}\left(D_{\alpha}\right)=\alpha, \operatorname{nacc}\left(D_{\alpha}\right) \subseteq G\right\} .
$$

It thus follows from [BR19a, Lemma 3.7] and [BR19c, Lemma 3.14] that $\mathrm{P}^{-}\left(\kappa, \kappa,{ }^{\Omega} \sqsubseteq\right.$, $\kappa,\{\Omega\}, 2,<\infty)$ holds. As $S \triangle \Omega$ is finite, it follows that $\mathrm{P}^{-}\left(\kappa, \kappa,{ }^{S} \sqsubseteq, \kappa,\{S\}, 2,<\infty\right)$ hold, as well.

Corollary 4.27. Suppose that $E$ is a nonreflecting stationary subset of a strongly inaccessible cardinal $\kappa$ and $\diamond(E)$ holds. Then, for every nonreflecting stationary $S \subseteq \kappa, \mathrm{P}^{-}\left(\kappa, \kappa, \sqsubseteq^{*}, 1,\{S\}, 2\right)$ holds.

Proof. Let $S \subseteq \kappa$ be stationary and nonreflecting. Then $E \cup S$ is also stationary and nonreflecting and $\diamond(E \cup S)$ holds, so that by Theorem $4.26(1), \mathrm{P}^{-}(\kappa, \kappa$, 
$\left.E \cup S_{\sqsubseteq}, 1,\{E \cup S\}, 2, \kappa\right)$ holds. In particular, we may fix a sequence $\overrightarrow{\mathcal{C}}=\left\langle\mathcal{C}_{\alpha}\right.$ $\alpha<\kappa\rangle$ witnessing $\mathrm{P}^{-}\left(\kappa, \kappa,{ }^{S} \sqsubseteq, 1,\{\kappa\}, 2,1\right)$. By ${ }^{S} \sqsubseteq$-coherence of $\overrightarrow{\mathcal{C}}$, we may assume that $\left|\mathcal{C}_{\alpha}\right|=1$ for every $\alpha \in S$. So, by [BR19c, Lemma 3.8] with $(\mu, \nu):=(\kappa, 2)$, $\mathrm{P}^{-}\left(\kappa, \kappa, \sqsubseteq^{*}, 1,\{S\}, 2,1\right)$ holds. Then, by Theorem $4.16(1), \mathrm{P}^{-}\left(\kappa, \kappa, \sqsubseteq^{*}, 1,\{S\}, 2\right)$ holds, as well.

Our next result about strongly inaccessible cardinals forms the core of Theorem B:

Theorem 4.28. Suppose that $\kappa$ is a strongly inaccessible cardinal, and there exists a sequence $\left\langle A_{\alpha} \mid \alpha \in S\right\rangle$ such that:

- $S$ is a nonreflecting stationary subset of $E_{>\omega}^{\kappa}$;

- For every $\alpha \in S, A_{\alpha}$ is a cofinal subset of $\alpha$;

- For every cofinal $B \subseteq \kappa$, there exists $\alpha \in S$ for which

$$
\left\{\delta<\alpha \mid \min \left(A_{\alpha} \backslash(\delta+1)\right) \in B\right\}
$$

is stationary in $\alpha$.

Then $\mathrm{P}^{-}\left(\kappa, \kappa,{ }^{S} \sqsubseteq, 1,\{S\}, 2\right)$ holds.

Proof. As $S$ is nonreflecting, for every $\alpha \in S$, we may pick a club subset $B_{\alpha}$ of $\alpha$ with $\operatorname{acc}\left(B_{\alpha}\right) \cap S=\emptyset$. Now, for every $\alpha \in S$, let $\mathcal{C}_{\alpha}:=\left\{C_{\alpha}\right\}$, where $C_{\alpha}$ denotes the closure in $\alpha$ of $\left\{\min \left(A_{\alpha} \backslash(\delta+1)\right) \mid \delta \in B_{\alpha}\right\}$. For every $\alpha \in \operatorname{acc}(\kappa) \backslash S$, let $\mathcal{C}_{\alpha}$ consist of all clubs $C$ in $\alpha$ such that $\operatorname{acc}(C) \cap S=\emptyset$. As $S$ is nonreflecting, $\kappa$ is strongly inaccessible, and $\operatorname{acc}\left(C_{\alpha}\right) \subseteq \operatorname{acc}\left(B_{\alpha}\right)$ for every $\alpha \in S$, it follows that $\overrightarrow{\mathcal{C}}:=\left\langle\mathcal{C}_{\alpha}\right.$ $\alpha<\kappa\rangle$ is a $\mathrm{P}^{-}\left(\kappa, \kappa,{ }^{S} \sqsubseteq, \ldots\right)$-sequence. To see that $\overrightarrow{\mathcal{C}}$ witnesses Clause (iv) of Theorem 4.15 with $(\xi, \mu, \mathcal{R}, \theta, \mathcal{S}, \nu):=\left(\kappa, \kappa,{ }^{S} \sqsubseteq, 1,\{S\}, 2\right)$, consider any given club $D \subseteq \kappa$ and cofinal $B \subseteq \kappa$. By Lemma 3.3. fix a cofinal subset $B^{\prime}$ of $B$ that is $D$ separated. By the hypothesis, fix $\alpha \in S$ such that $\left\{\delta<\alpha \mid \min \left(A_{\alpha} \backslash(\delta+1)\right) \in B^{\prime}\right\}$ is stationary in $\alpha$. In particular, $\sup \left(B^{\prime} \cap \alpha\right)=\alpha$, so that also $\sup (D \cap \alpha)=\alpha$, and it follows that $D \cap \alpha$ is a club in $\alpha$, and so is $D \cap B_{\alpha}$. Thus, the set $\left\{\delta \in D \cap B_{\alpha} \mid\right.$ $\left.\min \left(A_{\alpha} \backslash(\delta+1)\right) \in B^{\prime}\right\}$ is stationary in $\alpha$. As $\alpha \in S$, we infer that $\mathcal{C}_{\alpha}=\left\{C_{\alpha}\right\}$ (in particular, $\left.\left|\mathcal{C}_{\alpha}\right|=1\right)$, and for every $\delta \in B_{\alpha}, \min \left(C_{\alpha} \backslash(\delta+1)\right)=\min \left(A_{\alpha} \backslash(\delta+1)\right)$, yielding, in particular, $\sup \left\{\delta \in D \cap \alpha \mid \min \left(C_{\alpha} \backslash(\delta+1)\right) \in B\right\}=\alpha$. Thus, by Theorem 4.15(i), we obtain $\mathrm{P}^{-}\left(\kappa, \kappa,{ }^{S} \sqsubseteq, 1,\{S\}, 2,<\omega\right)$, as sought.

The proof of Lemma 4.20 makes clear that the $\diamond(\kappa)$ hypothesis in BR19c, Lemma 3.8] may be reduced to $\mathbf{s}(\kappa)$ together with $\kappa^{\aleph_{1}}=\kappa$, or together with $\kappa^{\aleph_{0}}=\kappa$ provided that $S$ concentrates on points of uncountable cofinality. In addition, the proof of Theorem 4.16(1) goes through also for $\mu=\kappa^{+}$. Therefore, we arrive at the following conclusion.

Lemma 4.29. Suppose that:

- $\mu \leq \kappa^{+}$and $\nu<\kappa$ are cardinals;

- $\overrightarrow{\mathcal{C}}=\left\langle\mathcal{C}_{\alpha} \mid \alpha<\kappa\right\rangle$ witnesses $\mathrm{P}^{-}\left(\kappa, \mu, \sqsubseteq^{*}, 1,\{\kappa\}, \mu, 1\right)$;

- $S$ is some stationary subset of $\left\{\alpha \in E_{>\omega}^{\kappa}|| \mathcal{C}_{\alpha} \mid<\nu\right\}$;

- $\kappa^{\aleph_{0}}=\kappa$ and $\mathbf{p}(\kappa)$ holds.

Then $\mathrm{P}^{-}\left(\kappa, \mu, \sqsubseteq^{*}, 1,\{S\}, \nu\right)$ holds.

Corollary 4.30. Suppose $\kappa^{\aleph_{0}}=\kappa$, and $\mathbf{\$}(E)$ holds for a nonreflecting stationary $E \subseteq \kappa$.

(1) For every $\mu \leq \kappa^{+}$and every stationary $S \subseteq E_{>\omega}^{\kappa}$ such that there is a $\mathrm{P}^{-}\left(\kappa, \mu,{ }^{E \cup S} \sqsubseteq^{*}, \ldots\right)$-sequence, $\mathrm{P}^{-}\left(\kappa, \mu, \sqsubseteq^{*}, 1,\{S\}, 2\right)$ holds;

(2) For every nonreflecting stationary $S \subseteq E_{>\omega}^{\kappa}, \mathrm{P}^{-}\left(\kappa, \kappa^{+}, \sqsubseteq^{*}, 1,\{S\}, 2\right)$ holds. 
Proof. We shall only prove Clause (1), as Clause (2) easily follows from it.

Suppose that $S \subseteq E_{>\omega}^{\kappa}$ is stationary, and there exists a $\mathrm{P}^{-}\left(\kappa, \mu,{ }^{E \cup S} \complement^{*}, \ldots\right)$ sequence, $\left\langle\mathcal{C}_{\alpha}^{0} \mid \alpha<\kappa\right\rangle$. Clearly, we may assume that $\left|\mathcal{C}_{\alpha}^{0}\right|=1$ for all $\alpha \in E \cup S$.

By the same proof of Corollary 4.22, there exists a stationary $\Omega \subseteq E$ and a $\mathrm{P}^{-}\left(\kappa, \mu,{ }^{\Omega} \sqsubseteq^{*}, 1,\{\Omega\}, 2,1\right)$-sequence $\overrightarrow{\mathcal{C}}=\left\langle\mathcal{C}_{\alpha} \mid \alpha<\kappa\right\rangle$ with $\left|\mathcal{C}_{\alpha}\right| \leq\left|\mathcal{C}_{\alpha}^{0}\right|$ for all $\alpha<\kappa$. In particular, $\overrightarrow{\mathcal{C}}$ is a $\mathrm{P}^{-}\left(\kappa, \mu, \sqsubseteq^{*}, 1,\{\kappa\}, \mu, 1\right)$-sequence with $S \subseteq\left\{\alpha \in E_{>\omega}^{\kappa}|| \mathcal{C}_{\alpha} \mid<\right.$ $2\}$. Then, by Lemma 4.29] $\mathrm{P}^{-}\left(\kappa, \mu, \Xi^{*}, 1,\left\{S^{\prime}\right\}, 2\right)$ holds.

We conclude this subsection, pointing out a few additional connections between instances of $\mathrm{P}^{-}(\kappa, \ldots)$ and the existence of nonreflecting stationary subsets of $\kappa$.

Proposition 4.31. Assume $\chi \in \operatorname{Reg}(\kappa), \min \{\theta, \sigma\}>0$ and $\mathcal{R}$ as in Example 4.5.

(1) Let $\xi<\kappa$. For any $S \subseteq E_{\geq \chi}^{\kappa}, \mathrm{P}_{\xi}^{-}(\kappa, \kappa, \chi \sqsubseteq, 1,\{S\}, 2,1)$ entails the existence of a nonreflecting stationary subset of $S$. In addition, for any $S \subseteq \kappa$, $\mathrm{P}_{\xi}^{-}\left(\kappa, \kappa, \sqsubseteq_{\chi}, 1,\{S\}, 2,1\right)$ entails the existence of stationary subset $\Omega \subseteq S$ such that, for every $\alpha \in E_{\geq \max \left\{\chi, \omega_{1}\right\}}^{\kappa}, \Omega \cap \alpha$ is nonstationary in $\alpha$.

(2) For any $\sigma \in \operatorname{Reg}(\kappa), \mathrm{P}^{-}\left(\kappa, 2, \sigma \sqsubseteq, 1,\left\{E_{\geq \sigma}^{\kappa}\right\}, 2, \sigma\right)$ entails the existence of a nonreflecting stationary subset of $E_{\sigma}^{\kappa}$.

(3) $\mathrm{P}_{\xi}^{-}\left(\kappa, \mu,{ }^{\Omega} \mathcal{R}, \theta,\{\Omega\}, \nu, \sigma\right)$ implies that $\Omega$ is a nonreflecting stationary subset of $\kappa$ and that $\mathrm{P}_{\xi}^{-}\left(\kappa, \mu,{ }^{\Omega} \mathcal{R}, \theta,\{\Omega\}, \nu^{\prime}, \sigma\right)$ holds with $\nu^{\prime}=2$.

(4) If $\kappa=\lambda^{+}$for a regular cardinal $\lambda$, then $\mathrm{P}_{\lambda}^{-}(\kappa, \mu, \mathcal{R}, \theta, \mathcal{S}, \nu, \sigma)$ is equivalent to $\mathrm{P}^{-}\left(\kappa, \mu,{ }_{\lambda}^{\kappa} \mathcal{R}, \theta, \mathcal{S}, \nu, \sigma\right)$.

(5) If $\kappa=\lambda^{+}$for a regular cardinal $\lambda$, then, for every $\mathcal{S} \subseteq \mathcal{P}\left(E_{\lambda}^{\kappa}\right), \mathrm{P}_{\lambda}^{-}(\kappa, \mu, \mathcal{R}$, $\theta, \mathcal{S}, \nu, \sigma)$ is equivalent to $\mathrm{P}_{\lambda}^{-}(\kappa, \mu, \mathcal{R}, \theta, \mathcal{S}, 2, \sigma)$.

(6) If $\kappa=\lambda^{+}$for an infinite cardinal $\lambda$, then, for every $\theta \geq \lambda, \mathrm{P}_{\lambda}^{-}(\kappa, \mu, \mathcal{R}, \theta,\{\kappa\}$, $\nu, \sigma)$ is equivalent to $\mathrm{P}_{\lambda}^{-}\left(\kappa, \mu, \mathcal{R}, \theta,\left\{E_{\mathrm{cf}(\lambda)}^{\kappa}\right\}, 2, \sigma\right)$.

Proof. (1) The easy argument may be extracted from the proof of (2) $\Longrightarrow(1)$ of [BR19c, Corollary 3.4].

(2) The proof is a straight-forward generalization of the proof of Lam17a, Theorem 4.1].

(3) From $\mathrm{P}_{\xi}^{-}(\kappa, \cdots, \theta,\{\Omega\}, \cdot, \sigma)$ with $\min \{\theta, \sigma\}>0$, we infer that $\Omega$ is stationary. Now, for every $\alpha \in E_{>\omega}^{\kappa}$, we may pick a club $C \in \mathcal{C}_{\alpha}$, and so by ${ }^{\Omega} \mathcal{R}$, we know that the club $\operatorname{acc}(C)$ is disjoint from $\Omega$, so that $\Omega \cap \alpha$ is nonstationary in $\alpha$. In effect, for every $\alpha \in \Omega$, we may replace $\mathcal{C}_{\alpha}$ by some singleton subset of itself, and then see that $\mathrm{P}_{\xi}^{-}\left(\kappa, \mu,{ }^{\Omega} \mathcal{R}, \theta,\{\Omega\}, 2, \sigma\right)$ holds, as well.

(4) This is obvious.

(5) By Clauses (3) and (4).

(6) By Clause (3) and [BR19c, Remarks 3.22].

4.4. Hitting on a club. Let $\mathrm{NS}_{\kappa}$ denote the nonstationary ideal over $\kappa$, so that $\mathrm{NS}_{\kappa}^{+}$forms the collection of all stationary subsets of $\kappa$. For every stationary $T \subseteq \kappa$, one denotes $\mathrm{NS}_{\kappa}^{+}\left\lceil T:=\mathrm{NS}_{\kappa}^{+} \cap \mathcal{P}(T)\right.$.

The following result shows that hitting on a club enables increasing the number of sets being hit simultaneously to the maximal possible value. It is used in the proof of [BR19b, Corollary 3.6], and in the justification of [BR19b, Remark ii. following Definition 3.3] and [Lam17a, Theorem 1.10(2)].

Lemma 4.32. Suppose that $\mathcal{S}=\mathrm{NS}_{\kappa}^{+} \uparrow T$ for some stationary $T \subseteq \kappa$. Then $\mathrm{P}_{\xi}^{-}(\kappa, \mu, \mathcal{R}, 1, \mathcal{S}, \nu, \sigma)$ is equivalent to $\mathrm{P}_{\xi}^{-}(\kappa, \mu, \mathcal{R}, \theta, \mathcal{S}, \nu, \sigma)$ with $\theta=\kappa$.

Proof. We focus on the forward implication. Fix a sequence $\overrightarrow{\mathcal{C}}=\left\langle\mathcal{C}_{\alpha} \mid \alpha<\kappa\right\rangle$ witnessing $\mathrm{P}_{\xi}^{-}(\kappa, \mu, \mathcal{R}, 1, \mathcal{S}, \nu, \sigma)$. In particular, for every cofinal $B \subseteq \kappa$, if we let 
$G(B)$ denote the set of all $\alpha<\kappa$ such that $\left|\mathcal{C}_{\alpha}\right|<\nu$, and for all $C \in \mathcal{C}_{\alpha}$,

$$
\sup \left\{\gamma \in C \mid \operatorname{succ}_{\sigma}(C \backslash \gamma) \subseteq B\right\}=\alpha,
$$

then, for every $S \in \mathcal{S}, G(B) \cap S$ is stationary. Recalling that $\mathcal{S}=\{S \subseteq T \mid$ $T$ is stationary , this means that $T \backslash G(B)$ is nonstationary.

Claim 4.32.1. $\overrightarrow{\mathcal{C}}$ witnesses $\mathrm{P}_{\xi}^{-}(\kappa, \mu, \mathcal{R}, \kappa, \mathcal{S}, \nu, \sigma)$, as well.

Proof. Suppose that $\left\langle B_{i} \mid i<\kappa\right\rangle$ is a given sequence of cofinal subsets of $\kappa$. For every $i<\kappa$, let us fix a club $D_{i} \subseteq \kappa$ such that $D_{i} \cap T \subseteq G\left(B_{i}\right)$. Consider the club $D:=\operatorname{acc}(\kappa) \cap\left(\triangle_{i<\kappa} D_{i}\right)$. Let $\alpha \in D \cap T$ be arbitrary. By $\alpha \in D_{0} \cap T$, we infer $\left|\mathcal{C}_{\alpha}\right|<\nu$. Let $C \in \mathcal{C}_{\alpha}$ be arbitrary. Given $i<\alpha$, we have $\alpha \in D_{i} \cap T$, and hence

$$
\sup \left\{\gamma \in C \mid \operatorname{succ}_{\sigma}(C \backslash \gamma) \subseteq B_{i}\right\}=\alpha
$$

as required.

This completes the proof.

Remark 4.33. Recalling Conventions 4.6 and 4.14, the principle $\mathrm{P}^{*}(T, \xi)$ of $[\mathrm{BR} 19 \mathrm{~b}$, Definition 3.3] is nothing but $\mathrm{P}_{\xi}^{-}\left(\kappa, \infty, \sqsubseteq, 1, \mathrm{NS}_{\kappa}^{+} \uparrow T, 2,<\infty\right)$. In particular, $\mathrm{P}^{*}(T, \kappa)$ entails $\mathrm{P}^{-}(\kappa, \kappa, \sqsubseteq, \kappa,\{\kappa\}, 2,1)$, which, by the results of Subsection 6.7 entails the existence of a free $\kappa$-Souslin tree. This justifies remark (ii) after Definition 3.3 of BR19b.

Consider a regular infinite cardinal $\lambda$ and some stationary set $S \subseteq E_{\lambda}^{\lambda^{+}}$. Similar to the fact that $\diamond(S)$ implies $\mathrm{P}_{\lambda}^{-}\left(\lambda^{+}, 2, \lambda \sqsubseteq, 1,\{S\}, 2, \sigma\right)$ (cf. BR17a, Theorem $5.1(2)])$, it is the case that $\diamond^{*}(S)$ implies $\mathrm{P}_{\lambda}^{-}\left(\lambda^{+}, 2,{ }_{\lambda} \sqsubseteq, 1, \mathrm{NS}_{\lambda^{+}}^{+}\lceil S, 2, \sigma) 20\right.$ To prove this, let us first dispose of the following:

Proposition 4.34. Suppose $\alpha$ is some limit ordinal, and $\mathcal{A}$ is a collection of $\operatorname{cf}(\alpha)$ many cofinal subsets of $\alpha$. Then there exists a club $C$ in $\alpha$ of order-type $\operatorname{cf}(\alpha)$ satisfying the following. For all $A \in \mathcal{A}$ and $\sigma<\operatorname{cf}(\alpha)$ :

$$
\sup \left\{\gamma \in C \mid \operatorname{succ}_{\sigma}(C \backslash \gamma) \subseteq A\right\}=\alpha .
$$

Proof. Let $\lambda:=\operatorname{cf}(\alpha)$. Let $\left\langle\alpha_{i} \mid i<\lambda\right\rangle$ be the strictly increasing enumeration of a club in $\alpha$. Fix a surjection $g: \lambda \rightarrow \mathcal{A}$ such that for all $\sigma<\lambda$ and $A \in \mathcal{A}$, the set $\left\{k<\lambda \mid g^{\prime \prime}(k, k+\sigma)=\{A\}\right\}$ is cofinal in $\lambda$. Now, recursively construct a strictly increasing and continuous sequence $\left\langle\gamma_{i} \mid i<\lambda\right\rangle$ such that, for all $i<\lambda, \gamma_{i}>\alpha_{i}$ and $\gamma_{i+1} \in g(i)$. Evidently, $C:=\left\{\gamma_{i} \mid i<\lambda\right\}$ is as sought.

Clause (2) of the next theorem is used in the proof of [BR19b, Proposition 3.10].

Theorem 4.35. Suppose $\lambda$ is any regular infinite cardinal, and $S \subseteq E_{\lambda}^{\lambda^{+}}$is a stationary subset such that $\diamond^{*}(S)$ holds. Set $\Omega:=E_{\lambda}^{\lambda^{+}}$. Then:

(1) $\mathrm{P}_{\lambda}^{-}\left(\lambda^{+}, 2,{ }_{\lambda}^{\Omega} \sqsubseteq, 1, \mathrm{NS}_{\lambda^{+}}^{+} \uparrow S, 2,<\infty\right)$ holds.

(2) If, in addition, $\lambda^{<\lambda}=\lambda$, then $\mathrm{P}_{\lambda}^{-}\left(\lambda^{+}, \infty,{ }^{\Omega} \sqsubseteq, 1, \mathrm{NS}_{\lambda^{+}}^{+} \uparrow S, 2,<\infty\right)$ holds.

Proof. (1) Fix a sequence $\overrightarrow{\mathcal{A}}=\left\langle\mathcal{A}_{\alpha} \mid \alpha \in S\right\rangle$ witnessing $\diamond^{*}(S)$. Without loss of generality, we may assume that, for all $\alpha \in S, \mathcal{A}_{\alpha}^{\prime}:=\left\{A \in \mathcal{A}_{\alpha} \mid \sup (A)=\alpha\right\}$ is of size $\lambda$. We define a sequence $\left\langle C_{\alpha} \mid \alpha<\lambda^{+}\right\rangle$, as follows:

- For each $\alpha \in \lambda^{+} \backslash S$, pick a closed subset $C_{\alpha}$ of $\alpha$ with $\sup \left(C_{\alpha}\right)=\sup (\alpha)$ and $\operatorname{otp}\left(C_{\alpha}\right)=\operatorname{cf}(\alpha)$.

- For each $\alpha \in S$, let $C_{\alpha}$ be given by Proposition 4.34 when fed with $\mathcal{A}_{\alpha}^{\prime}$.

\footnotetext{
${ }^{20}$ For the definition of $\diamond^{*}(S)$, see Rin11a, Definition 1.3].
} 
Now, set $\overrightarrow{\mathcal{C}}=\left\langle\mathcal{C}_{\alpha} \mid \alpha<\lambda^{+}\right\rangle$where $\mathcal{C}_{\alpha}:=\left\{C_{\alpha}\right\}$ for all $\alpha<\lambda^{+}$. Then $\overrightarrow{\mathcal{C}}$ is a $\mathrm{P}_{\lambda}^{-}\left(\lambda^{+}, 2,{ }_{\lambda}^{\Omega} \sqsubseteq, \ldots\right)$-sequence, since, for every $\alpha \in \operatorname{acc}\left(\lambda^{+}\right), C_{\alpha}$ is a club subset of $\alpha$ of order-type $\leq \lambda$, so that ${ }_{\lambda}^{\Omega} \sqsubseteq$-coherence is satisfied vacuously.

To see that $\overrightarrow{\mathcal{C}}$ witnesses $\mathrm{P}_{\lambda}^{-}\left(\lambda^{+}, 2,{ }_{\lambda}^{\Omega} \sqsubseteq, 1, \mathrm{NS}_{\lambda^{+}}^{+}\lceil S, 2,<\infty)\right.$, consider any cofinal set $A \subseteq \lambda^{+}$. By our choice of $\overrightarrow{\mathcal{A}}$, we can fix a club $D \subseteq \lambda^{+}$such that, for every $\alpha \in D \cap S, A \cap \alpha \in \mathcal{A}_{\alpha}$. Consider the club $E:=\operatorname{acc}^{+}(A) \cap D$. For any $\alpha \in E \cap S$, we obtain $A \cap \alpha \in \mathcal{A}_{\alpha}^{\prime}$, so that by our choice of $C_{\alpha}$ it follows that, for all $\sigma<\lambda$,

$$
\sup \left\{\gamma \in C \mid \operatorname{succ}_{\sigma}(C \backslash \gamma) \subseteq A\right\}=\alpha,
$$

as sought.

(2) Let $\overrightarrow{\mathcal{C}}=\left\langle\mathcal{C}_{\alpha} \mid \alpha<\lambda^{+}\right\rangle$be given by Clause (1). Define $\overrightarrow{\mathcal{D}}=\left\langle\mathcal{D}_{\alpha} \mid \alpha<\lambda^{+}\right\rangle$as follows.

- For all $\alpha \leq \lambda$, let $\mathcal{D}_{\alpha}:=\{\alpha\}$.

- For all $\alpha \in E_{<\lambda}^{\lambda^{+}} \backslash \lambda$, let $\mathcal{D}_{\alpha}$ be the collection of all clubs $d$ in $\alpha$ such that $\min (d) \geq \lambda$ and $|d|<\lambda$.

- For all $\alpha \in E_{\lambda}^{\lambda^{+}} \backslash\{\lambda\}$, let $\mathcal{D}_{\alpha}:=\left\{C \backslash \lambda \mid C \in \mathcal{C}_{\alpha}\right\}$.

Then $\overrightarrow{\mathcal{D}}$ witnesses $\mathrm{P}_{\lambda}^{-}\left(\lambda^{+}, \infty,{ }^{\Omega} \sqsubseteq, 1, \mathrm{NS}_{\lambda^{+}}^{+} \mid S, 2,<\infty\right)$.

4.5. Improving the coherence. In this subsection, we show that we can improve the coherence of a sequence witnessing the proxy principle by inserting all necessary initial segments of the clubs into the relevant collections, as long as we allow the sequence to grow wide enough. The main result here is the following:

Corollary 4.36. $\mathrm{P}_{\xi}^{-}\left(\kappa, \kappa, \sqsubseteq^{*}, \theta, \mathcal{S}, \kappa, \sigma\right)$ is equivalent to $\mathrm{P}_{\xi}^{-}(\kappa, \kappa, \sqsubseteq, \theta, \mathcal{S}, \kappa, \sigma)$.

Proof. By Theorem 4.39 below, using $(\Omega, \chi, \nu):=(\emptyset, 0, \kappa)$ and Remark 4.38

The following notion will allow us to state the main result of this subsection in its most general form.

Definition 4.37. Let $\mathrm{P}_{\xi}^{-}\left(\kappa, \mu, \mathcal{R}, \theta, \mathcal{S}, \nu^{*}\right)$ be the assertion obtained by replacing Clause (1) of Definition 4.10 by the weaker property:

$\left(1^{*}\right)$ there exists $\mathcal{C} \in\left[\mathcal{C}_{\alpha}\right]^{<\nu}$ such that, for all $C \in \mathcal{C}_{\alpha}$, there is $C^{\prime} \in \mathcal{C}$ with $\sup \left(C \triangle C^{\prime}\right)<\alpha$.

Remark 4.38. Clearly, for any vector of parameters $\ldots$, we have:

$$
\mathrm{P}_{\xi}^{-}(\kappa, \mu, \varpi, \nu) \Longrightarrow \mathrm{P}_{\xi}^{-}\left(\kappa, \mu, \varpi, \nu^{*}\right) \Longrightarrow \mathrm{P}_{\xi}^{-}(\kappa, \mu, \varpi, \mu) .
$$

Note that setting $(\Omega, \theta, \nu, \sigma):=(\emptyset, 1, \kappa, 1)$ and $\mathcal{S}$ a singleton in the following Theorem provides the justification for [BR19b, Remark (i) following Definition 2.1].

Theorem 4.39. Suppose $\kappa$ is $(<\chi)$-closed and $\mathcal{S} \subseteq \mathcal{P}\left(E_{\geq \chi}^{\kappa}\right)$.

Then $\mathrm{P}_{\xi}^{-}\left(\kappa, \kappa,{ }_{\chi} \sqsubseteq^{*}, \theta, \mathcal{S}, \nu, \sigma\right)$ is equivalent to $\mathrm{P}_{\xi}^{-}\left(\kappa, \kappa,{ }^{\Omega} \sqsubseteq, \theta, \mathcal{S}, \nu^{*}, \sigma\right)$.

Proof. ( $\Longleftarrow)$ Fix a sequence $\left\langle\mathcal{C}_{\alpha} \mid \alpha<\kappa\right\rangle$ witnessing $\mathrm{P}_{\xi}^{-}\left(\kappa, \kappa,{ }^{\Omega} \sqsubseteq, \theta, \mathcal{S}, \nu^{*}, \sigma\right)$. For every $\alpha \in \operatorname{acc}(\kappa)$, pick a subset $\mathcal{C}_{\alpha}^{\bullet} \subseteq \mathcal{C}_{\alpha}$ of minimal cardinality such that, for all $C \in \mathcal{C}_{\alpha}$, there is $C^{\prime} \in \mathcal{C}_{\alpha}^{\bullet}$ with $\sup \left(C \triangle C^{\prime}\right)<\alpha$.

Recalling Convention 4.4, the sequence $\left\langle\mathcal{C}_{\alpha}^{\bullet} \mid \alpha<\kappa\right\rangle$ witnesses $\mathrm{P}_{\xi}^{-}\left(\kappa, \kappa,{ }^{\Omega} \sqsubseteq^{*}, \theta, \mathcal{S}\right.$, $\nu, \sigma)$, as seen by noting the following:

- For any $\alpha \in \operatorname{acc}(\kappa), C \in \mathcal{C}_{\alpha}^{\bullet}$, and $\bar{\alpha} \in \operatorname{acc}(C)$, we may pick $D \in \mathcal{C}_{\bar{\alpha}}$ with $D^{\Omega} \sqsubseteq C$, and then pick $D^{\prime} \in \mathcal{C}_{\bar{\alpha}}^{\bullet}$ with $\sup \left(D \triangle D^{\prime}\right)<\bar{\alpha}$. Thus $\sup \left((C \cap \bar{\alpha}) \triangle D^{\prime}\right)<\bar{\alpha}$, so that $D^{\prime} \Omega^{*} C$, as sought.

- By Definition 4.37, $\left|\mathcal{C}_{\alpha}^{\bullet}\right|<\nu$ wherever the hitting takes place. 
$(\Longrightarrow)$ Fix a sequence $\overrightarrow{\mathcal{C}}=\left\langle\mathcal{C}_{\delta} \mid \delta<\kappa\right\rangle$ witnessing $\mathrm{P}_{\xi}^{-}\left(\kappa, \kappa, \underset{\chi}{\chi} \sqsubseteq^{*}, \theta, \mathcal{S}, \nu, \sigma\right)$. Denote $\Sigma:=\bigcup_{\delta \in \operatorname{acc}(\kappa)} \mathcal{C}_{\delta}$, and for every $\alpha<\kappa$, denote $\mathcal{A}_{\alpha}:=\{C \cap \alpha \mid C \in \Sigma\}$ and $\mathcal{G}_{\alpha}:=\left\{c \in \mathcal{A}_{\alpha} \mid \sup (c)=\alpha\right\}$. Recalling Convention 4.4, let $\overrightarrow{\mathcal{D}}:=\left\langle\mathcal{D}_{\alpha} \mid \alpha<\kappa\right\rangle$ be determined by setting $\mathcal{D}_{\alpha}:=\mathcal{G}_{\alpha}$ for every $\alpha \in \operatorname{acc}(\kappa)$ (cf. [BR19c, Notation 2.4]). We shall show that $\overrightarrow{\mathcal{D}}$ witnesses $\mathrm{P}_{\xi}^{-}\left(\kappa, \kappa,{ }^{\Omega} \sqsubseteq, \theta, \mathcal{S}, \nu^{*}, \sigma\right)$.

Claim 4.39.1. Suppose $\alpha \in E_{\geq \chi}^{\kappa}$. Then every element $c \in \mathcal{G}_{\alpha}$ can be written as $c=(c \cap \beta) \cup(D \backslash \beta)$ for some $D \in \mathcal{C}_{\alpha}$ and $\beta<\alpha$, where $c \cap \beta \in \mathcal{A}_{\beta}$.

Proof. Consider any given $c \in \mathcal{G}_{\alpha}$. Fix $C \in \Sigma$ such that $c=C \cap \alpha$. As $c \in \mathcal{G}_{\alpha}$, we infer that $\sup (C \cap \alpha)=\alpha$, so that $\alpha \in \operatorname{acc}(C) \cup\{\sup (C)\}$. If $\alpha \in \operatorname{acc}(C)$, then by $\chi \sqsubseteq^{*}$-coherence and the fact that $\operatorname{cf}(\alpha) \geq \chi$, we can fix some $D \in \mathcal{C}_{\alpha}$ such that $D \sqsubseteq{ }^{*} C$. Otherwise, $\alpha=\sup (C)$, so that in fact $c=C \in \mathcal{C}_{\alpha}$; in this case we set $D:=C$. In either case, set $\beta:=\min \{\beta \mid D \backslash \beta \sqsubseteq C \backslash \beta\}$. As $D \sqsubseteq{ }^{*} C$, it is clear that $\beta$ is an ordinal $<\alpha$. Furthermore, as $\sup (D)=\sup (c)=\alpha$ and $c=C \cap \alpha$, it follows that $D \backslash \beta=c \backslash \beta$. Finally, notice that $c \cap \beta=C \cap \beta \in \mathcal{A}_{\beta}$, and it is clear that $c=(c \cap \beta) \cup(D \backslash \beta)$.

Claim 4.39.2. $\overrightarrow{\mathcal{D}}$ is a $\mathrm{P}_{\xi}^{-}\left(\kappa, \kappa,{ }^{\Omega} \sqsubseteq, \ldots\right)$-sequence satisfying $\mathcal{C}_{\alpha} \subseteq \mathcal{D}_{\alpha}$ for every $\alpha \in \operatorname{acc}(\kappa)$, and $\mathcal{D}_{\alpha}=\mathcal{C}_{\alpha}$ for every $\alpha \in \Omega \cap \operatorname{acc}(\kappa)$.

Proof. Consider any given $\alpha \in \operatorname{acc}(\kappa)$. It is clear that $\mathcal{C}_{\alpha} \subseteq \mathcal{D}_{\alpha}$, so that, in particular, $\mathcal{D}_{\alpha} \neq \emptyset$. Now, fix any given $c \in \mathcal{D}_{\alpha}$. By definition of $\mathcal{D}_{\alpha}$, we can fix some $C \in \Sigma$ such that $c=C \cap \alpha$ and $\sup (c)=\alpha$. In particular, $c \in \mathcal{K}(\kappa)$ and $\alpha_{c}=\alpha$. Furthermore, $\operatorname{otp}(c) \leq \operatorname{otp}(C) \leq \xi$. If, in addition, $\alpha \in \Omega$, then by ${ }_{\chi}^{\Omega} \sqsubseteq^{*}-$ coherence of $\overrightarrow{\mathcal{C}}$ we cannot have $\alpha \in \operatorname{acc}(C)$, so the only way to have $\sup (C \cap \alpha)=\alpha$ is if in fact $\alpha=\sup (C)$, meaning that $c=C \in \mathcal{C}_{\alpha}$.

Next, we verify ${ }^{\Omega} \sqsubseteq$-coherence: Consider arbitrary $\alpha \in \operatorname{acc}(\kappa), c \in \mathcal{D}_{\alpha}$, and $\bar{\alpha} \in \operatorname{acc}(c)$. Pick $C \in \Sigma$ such that $c=C \cap \alpha$. Then $\bar{\alpha} \in \operatorname{acc}(C)$, so that by ${ }_{\chi}^{\Omega} \sqsubseteq^{*}-$ coherence of $\overrightarrow{\mathcal{C}}$ we obtain $\bar{\alpha} \notin \Omega$. Clearly, $c \cap \bar{\alpha}=C \cap \bar{\alpha} \in \mathcal{A}_{\bar{\alpha}}$. By $\bar{\alpha} \in \operatorname{acc}(C)$, we moreover obtain $C \cap \bar{\alpha} \in \mathcal{D}_{\bar{\alpha}}$, and hence $c \cap \bar{\alpha} \in \mathcal{D}_{\bar{\alpha}}$.

It remains to show that $\left|\mathcal{D}_{\alpha}\right|<\kappa$ for every $\alpha \in \operatorname{acc}(\kappa)$. In fact, we shall prove, by induction on $\alpha$, the stronger result that $\left|\mathcal{A}_{\alpha}\right|<\kappa$ for every ordinal $\alpha<\kappa$. Thus, fix a given ordinal $\alpha<\kappa$, and assume that $\left|\mathcal{A}_{\gamma}\right|<\kappa$ for every ordinal $\gamma<\alpha$; we shall first show that $\left|\mathcal{G}_{\alpha}\right|<\kappa$, and then argue further that $\left|\mathcal{A}_{\alpha}\right|<\kappa$.

To see that $\left|\mathcal{G}_{\alpha}\right|<\kappa$, we consider several cases:

- If $\alpha=0$, then $\mathcal{G}_{\alpha}=\{\emptyset\}$ is a singleton.

- If $\alpha$ is a successor ordinal, then $\mathcal{G}_{\alpha}$ is empty.

Thus we may assume that $\alpha \in \operatorname{acc}(\kappa)$.

- Suppose $\alpha \in E_{<\chi}^{\kappa}$. Set $\eta:=\operatorname{cf}(\alpha)$, and fix an increasing sequence $\left\langle\gamma_{i}\right|$ $i<\eta\rangle$ of ordinals converging to $\alpha$. Define a function $\varphi: \mathcal{G}_{\alpha} \rightarrow \prod_{i<\eta} \mathcal{A}_{\gamma_{i}}$ by setting $\varphi(c):=\left\langle c \cap \gamma_{i} \mid i<\eta\right\rangle$ for every $c \in \mathcal{G}_{\alpha}$. For every $i<\eta$, we have $\left|\mathcal{A}_{\gamma_{i}}\right|<\kappa$ by the induction hypothesis, since $\gamma_{i}<\alpha$. Let $\lambda:=\sup _{i<\eta}\left|\mathcal{A}_{\gamma_{i}}\right|$. We infer from the regularity of $\kappa$ that $\lambda<\kappa$, and then since $\eta<\chi$, it follows from $(<\chi)$-closedness of $\kappa$ that $|\operatorname{Im}(\varphi)| \leq \lambda^{\eta}<\kappa$. As $c=\bigcup_{i<\eta}\left(c \cap \gamma_{i}\right)$ for every $c \in \mathcal{G}_{\alpha}$, it is clear that $\varphi$ is injective. Altogether, $\left|\mathcal{G}_{\alpha}\right|<\kappa$, as sought.

- Finally, suppose $\alpha \in E_{\geq \chi}^{\kappa}$. In this case, define a function

$$
\varphi: \mathcal{G}_{\alpha} \rightarrow \bigcup_{\beta<\alpha} \mathcal{A}_{\beta} \times \alpha \times \mathcal{C}_{\alpha}
$$

by setting $\varphi(c):=\left(c \cap \beta_{c}, \beta_{c}, D_{c}\right)$, where the representation $c=\left(c \cap \beta_{c}\right) \cup$ $\left(D_{c} \backslash \beta_{c}\right)$ is given by Claim 4.39.1. As $\left|\mathcal{A}_{\beta}\right|<\kappa$ for every ordinal $\beta<\alpha$, and 
$\left|\mathcal{C}_{\alpha}\right|<\kappa$ by our choice of $\overrightarrow{\mathcal{C}}$, it follows from regularity of $\kappa$ that $|\operatorname{Im}(\varphi)|<\kappa$.

Furthermore, it is clear that $\varphi$ is injective. Altogether, $\left|\mathcal{G}_{\alpha}\right|<\kappa$, as sought.

In all cases we have shown that $\left|\mathcal{G}_{\alpha}\right|<\kappa$. Of course, $\mathcal{G}_{\beta} \subseteq \mathcal{A}_{\beta}$ for every ordinal $\beta<\kappa$, so that our induction hypothesis implies that $\left|\mathcal{G}_{\beta}\right|<\kappa$ for every ordinal $\beta<\alpha$. We use these facts as we continue to show that $\left|\mathcal{A}_{\alpha}\right|<\kappa$.

Notice that every element $c \in \mathcal{A}_{\alpha}$ can be written as $c=\left(c \cap \beta_{c}\right) \cup\left(c \backslash \beta_{c}\right)$, where $\beta_{c}$ is some (possibly 0) limit ordinal $\leq \alpha, c \cap \beta_{c} \in \mathcal{G}_{\beta_{c}}$, and $c \backslash \beta_{c}$ is finite. To see this, consider any given $c \in \mathcal{A}_{\alpha}$. Define $\beta_{c}:=\min \left\{\beta|| c \backslash \beta \mid<\aleph_{0}\right\}$. As $c \subseteq \alpha$, it is clear that $\beta_{c}$ is an ordinal $\leq \alpha$. By minimality of $\beta_{c}$, it follows that $\beta_{c}$ is a limit ordinal (possibly 0) and $\sup \left(c \cap \beta_{c}\right)=\beta_{c}$. Fix $C \in \Sigma$ such that $c=C \cap \alpha$. Then $c \cap \beta_{c}=C \cap \beta_{c}$, so that, in particular, $c \cap \beta_{c} \in \mathcal{G}_{\beta_{c}}$. It is clear that $c=\left(c \cap \beta_{c}\right) \cup\left(c \backslash \beta_{c}\right)$, and by our definition of $\beta_{c}$ it is clear that $c \backslash \beta_{c}$ is finite.

Thus, to show that $\left|\mathcal{A}_{\alpha}\right|<\kappa$, define a function

$$
\pi: \mathcal{A}_{\alpha} \rightarrow \bigcup\left\{\mathcal{G}_{\beta} \times[\alpha]^{<\aleph_{0}} \mid \beta \in \operatorname{acc}(\alpha+1) \cup\{0\}\right\}
$$

by setting $\pi(c):=\left(c \cap \beta_{c}, c \backslash \beta_{c}\right)$, where the representation $c=\left(c \cap \beta_{c}\right) \cup\left(c \backslash \beta_{c}\right)$ is the one given above. Since $|\alpha|<\kappa$ and $\kappa$ is infinite, we infer that $\left|[\alpha]<\aleph_{0}\right|<\kappa$. Then, by regularity of $\kappa$ and the fact that $\left|\mathcal{G}_{\beta}\right|<\kappa$ for every ordinal $\beta \leq \alpha$, it follows that $|\operatorname{Im}(\pi)|<\kappa$. As $c=\left(c \cap \beta_{c}\right) \cup\left(c \backslash \beta_{c}\right)$ for every $c \in \mathcal{A}_{\alpha}$, it is clear that $\pi$ is injective. Altogether, $\left|\mathcal{A}_{\alpha}\right|<\kappa$, as sought.

Claim 4.39.3. Suppose $\left\langle B_{i} \mid i<\theta\right\rangle$ is a sequence of cofinal subsets of $\kappa$, and $S \in \mathcal{S}$. Then there exist stationarily many $\alpha \in S$ such that:

(1) there exists $\mathcal{C} \in\left[\mathcal{D}_{\alpha}\right]^{<\nu}$ such that, for all $D \in \mathcal{D}_{\alpha}$, there is $C \in \mathcal{C}$ with $\sup (D \triangle C)<\alpha$

(2) for all $D \in \mathcal{D}_{\alpha}$ and $i<\min \{\alpha, \theta\}, \sup \left\{\gamma \in D \mid \operatorname{succ}_{\sigma}(D \backslash \gamma) \subseteq B_{i}\right\}=\alpha$.

Proof. Let $S^{*}$ denote the set of all $\alpha \in S \cap \operatorname{acc}(\kappa)$ such that $\left|\mathcal{C}_{\alpha}\right|<\nu$ and, for all $C \in \mathcal{C}_{\alpha}$ and $i<\min \{\alpha, \theta\}, \sup \left\{\gamma \in C \mid \operatorname{succ}_{\sigma}(C \backslash \gamma) \subseteq B_{i}\right\}=\alpha$. By the choice of $\overrightarrow{\mathcal{C}}, S^{*}$ is a stationary subset of $E_{\geq \chi}^{\kappa}$. Consider any given $\alpha \in S^{*}$. Then, by Claims 4.39.2 and 4.39.1, $\mathcal{C}:=\mathcal{C}_{\alpha}$ witnesses Clause (1). Now, given $D \in \mathcal{D}_{\alpha}$ and $i<\min \{\alpha, \theta\}$, we first pick $C \in \mathcal{C}_{\alpha}$ such that $\sup (D \triangle C)<\alpha$. As $\alpha \in S^{*}$, we know that $\sup \left\{\gamma \in C \mid \operatorname{succ}_{\sigma}(C \backslash \gamma) \subseteq B_{i}\right\}=\alpha$. As $\sup (D \triangle C)<\alpha$, it is also the case that $\sup \left\{\gamma \in D \mid \operatorname{succ}_{\sigma}(D \backslash \gamma) \subseteq B_{i}\right\}=\alpha$.

Thus, $\overrightarrow{\mathcal{D}}$ witnesses $\mathrm{P}_{\xi}^{-}\left(\kappa, \kappa,{ }^{\Omega} \sqsubseteq, \theta, \mathcal{S}, \nu^{*}, \sigma\right)$.

Corollary 4.40. $\mathrm{P}_{\xi}^{-}\left(\kappa, \kappa,{ }^{\Omega} \sqsubseteq^{*}, \theta,\{\Omega\}, \kappa, \sigma\right)$ is equivalent to $\mathrm{P}_{\xi}^{-}\left(\kappa, \kappa,{ }^{\Omega} \sqsubseteq, \theta,\{\Omega\}, 2, \sigma\right)$.

Proof. Apply Theorem 4.39 followed by Proposition 4.31(3).

4.6. Indexed ladders. In this subsection, we consider indexed $\mathrm{P}_{\xi}^{-}\left(\kappa, \mu^{+}, \sqsubseteq, \ldots\right)-$ sequences for infinite cardinals $\mu<\kappa$. Our proof of Theorem A will go through the following concept.

Definition 4.41 ([Lam17b, Definition 6.3]). $\square^{\text {ind }}(\kappa, \mu)$ asserts the existence of a matrix $\left\langle C_{\alpha, i} \mid \alpha<\kappa, i(\alpha) \leq i<\mu\right\rangle$ such that for all $\alpha \in \operatorname{acc}(\kappa)$ :

- $i(\alpha)<\mu$, and, for all $i \in[i(\alpha), \mu), C_{\alpha, i}$ is a club in $\alpha$;

- for all $i \in[i(\alpha), \mu)$ and $\bar{\alpha} \in \operatorname{acc}\left(C_{\alpha, i}\right), i \geq i(\bar{\alpha})$ and $C_{\bar{\alpha}, i} \sqsubseteq C_{\alpha, i}$;

- $\left\langle C_{\alpha, i} \mid i(\alpha) \leq i<\mu\right\rangle$ is $\subseteq$-increasing with $\operatorname{acc}(\alpha)=\bigcup_{i \in[i(\alpha), \mu)} \operatorname{acc}\left(C_{\alpha, i}\right)$, and such that, for every club $D$ in $\kappa$, there exists $\alpha \in \operatorname{acc}(D)$ such that $D \cap \alpha \neq C_{\alpha, i}$ for all $i \in[i(\alpha), \mu)$.

Motivated by the preceding definition, we introduce the following strengthening of $\mathrm{P}_{\xi}^{-}\left(\kappa, \mu^{+}, \sqsubseteq, \ldots\right)$ (compare with Definition 4.2) . 
Definition 4.42. We say that $\left\langle\mathcal{C}_{\alpha} \mid \alpha<\kappa\right\rangle$ is a $\mathrm{P}_{\xi}^{-}\left(\kappa, \mu^{\text {ind }}, \sqsubseteq, \ldots\right)$-sequence iff there exists a sequence $\langle i(\alpha) \mid \alpha<\kappa\rangle$ of ordinals in $\mu$, such that, for every $\alpha \in \operatorname{acc}(\kappa)$, all of the following hold:

- $\mathcal{C}_{\alpha} \subseteq\left\{C \in \mathcal{K}(\kappa) \mid \operatorname{otp}(C) \leq \xi \& \alpha_{C}=\alpha\right\}$;

- there exists a canonical enumeration $\left\langle C_{\alpha, i} \mid i(\alpha) \leq i<\mu\right\rangle$ of $\mathcal{C}_{\alpha}$ (possibly with repetition);

- for all $i \in[i(\alpha), \mu)$ and $\bar{\alpha} \in \operatorname{acc}\left(C_{\alpha, i}\right), i \geq i(\bar{\alpha})$ and $C_{\bar{\alpha}, i} \sqsubseteq C_{\alpha, i}$;

- $\left\langle\operatorname{acc}\left(C_{\alpha, i}\right) \mid i(\alpha) \leq i<\mu\right\rangle$ is $\subseteq$-increasing with $\operatorname{acc}(\alpha)=\bigcup_{i \in[i(\alpha), \mu)} \operatorname{acc}\left(C_{\alpha, i}\right)$.

We will need an adaptation of our wide-club-guessing lemma [BR19a, Lemma 2.5] to the context of indexed sequences.

Lemma 4.43. Suppose that $\aleph_{1} \leq \mu^{+}<\kappa$, and $\left\langle\mathcal{C}_{\alpha} \mid \alpha<\kappa\right\rangle$ is a $\mathrm{P}_{\xi}^{-}\left(\kappa, \mu^{\text {ind }}, \sqsubseteq, \ldots\right)$ sequence satisfying that, for every club $D \subseteq \kappa$, there exists $\alpha \in \operatorname{acc}(D)$ with $D \cap \alpha \notin$ $\mathcal{C}_{\alpha}$. Suppose $S \subseteq \kappa$ is stationary.

Then there is a $\mathrm{P}_{\xi}^{-}\left(\kappa, \mu^{\text {ind }}, \sqsubseteq, \ldots\right)$-sequence $\left\langle\mathcal{C}_{\alpha}^{\bullet} \mid \alpha<\kappa\right\rangle$ with the additional property that for every club $E \subseteq \kappa$, there exist stationarily many $\alpha \in S$ such that, for all $C \in \mathcal{C}_{\alpha}^{\bullet}, \sup (\operatorname{nacc}(C) \cap E)=\alpha$.

Proof. Fix a sequence $\langle i(\alpha) \mid \alpha<\kappa\rangle$ of ordinals in $\mu$ as in Definition 4.42. For every club $D \subseteq \kappa$, we define a map $\Phi_{D}: \mathcal{K}(\kappa) \rightarrow \mathcal{K}(\kappa)$ via

$$
\Phi_{D}(x):= \begin{cases}x \backslash \sup (D \cap \sup (x)), & \text { if } \sup (D \cap \sup (x))<\sup (x) ; \\ \{\sup (D \cap \eta) \mid \eta \in x \& \eta>\min (D)\}, & \text { if } \sup (D \cap \sup (x))=\sup (x) .\end{cases}
$$

By [BR19a, Lemma 2.2], $\Phi_{D}$ is a postprocessing function. Furthermore, by BR19a, Claim 2.5.1], we may fix a club $D \subseteq \kappa$ such that, for every club $E \subseteq \kappa$, there exists $\alpha \in S$ with $\sup \left(\operatorname{nacc}\left(\Phi_{D}(C)\right) \cap E\right)=\alpha$ for all $C \in \mathcal{C}_{\alpha}$. Let $\pi: \kappa \leftrightarrow D$ denote the order-preserving bijection. Now, for every $\alpha \in \operatorname{acc}(\kappa)$, let $j(\alpha):=i(\pi(\alpha))$ and set

$$
C_{\alpha, i}^{\bullet}:=\pi^{-1}\left[\Phi_{D}\left(C_{\pi(\alpha), i}\right)\right]
$$

for every $i \in[j(\alpha), \mu)$. Put $\mathcal{C}_{\alpha}^{\bullet}:=\left\{C_{\alpha, i}^{\bullet} \mid j(\alpha) \leq i<\mu\right\}$.

Claim 4.43.1. Let $\alpha \in \operatorname{acc}(\kappa)$ and $i \in[j(\alpha), \mu)$. Then:

(1) $C_{\alpha, i}^{\bullet}$ is a club in $\alpha$ of order-type $\leq \xi$;

(2) for all $\bar{\alpha} \in \operatorname{acc}\left(C_{\alpha, i}^{\bullet}\right), i \geq j(\bar{\alpha})$ and $C_{\bar{\alpha}, i}^{\bullet} \sqsubseteq C_{\alpha, i}^{\bullet}$.

Proof. (1) As $\alpha \in \operatorname{acc}(\kappa), \pi(\alpha) \in \operatorname{acc}(D)$, and hence the definition of $\Phi_{D}$ implies that $\Phi_{D}\left(C_{\pi(\alpha), i}\right)$ is a club in $\pi(\alpha)$ which is a subset of $D$. So $C_{\alpha, i}^{\bullet}=$ $\pi^{-1}\left[\Phi_{D}\left(C_{\pi(\alpha), i}\right)\right]$ is a club in $\alpha$ of order-type otp $\left(C_{\pi(\alpha), i}\right) \leq \xi$.

(2) Suppose $\bar{\alpha} \in \operatorname{acc}\left(C_{\alpha, i}^{\bullet}\right)$. Then $\bar{\alpha} \in \operatorname{acc}\left(\pi^{-1}\left[\Phi_{D}\left(C_{\pi(\alpha), i}\right)\right]\right)$, so that $\pi(\bar{\alpha}) \in$ $\operatorname{acc}\left(\Phi_{D}\left(C_{\pi(\alpha), i}\right)\right)=\operatorname{acc}\left(C_{\pi(\alpha), i}\right) \cap \operatorname{acc}(D)$. In particular, $i \geq i(\pi(\bar{\alpha}))=j(\bar{\alpha})$ and $C_{\pi(\bar{\alpha}), i} \sqsubseteq C_{\pi(\alpha), i}$. As $\Phi_{D}$ is a postprocessing function, $\Phi_{D}\left(C_{\pi(\bar{\alpha}), i}\right) \sqsubseteq \Phi_{D}\left(C_{\pi(\alpha), i}\right)$, and hence $C_{\bar{\alpha}, i}^{\bullet} \sqsubseteq C_{\alpha, i}^{\bullet}$.

Claim 4.43.2. Let $\alpha \in \operatorname{acc}(\kappa)$. Then $\left\langle\operatorname{acc}\left(C_{\alpha, i}^{\bullet}\right) \mid j(\alpha) \leq i<\mu\right\rangle$ is $\subseteq$-increasing with $\operatorname{acc}(\alpha)=\bigcup_{i \in[j(\alpha), \mu)} \operatorname{acc}\left(C_{\alpha, i}^{\bullet}\right)$.

Proof. It is clear that for any two clubs $C, C^{\prime}$ in $\pi(\alpha), C \subseteq C^{\prime} \Longrightarrow \Phi_{D}(C) \subseteq$ $\Phi_{D}\left(C^{\prime}\right)$. Consequently, $\left\langle\operatorname{acc}\left(C_{\alpha, i}^{\bullet}\right) \mid j(\alpha) \leq i<\mu\right\rangle$ is $\subseteq$-increasing. Finally, for any $\bar{\alpha} \in \operatorname{acc}(\alpha)$, as $\pi(\bar{\alpha}) \in \operatorname{acc}(\pi(\alpha))$, we may find a large enough $i \geq i(\pi(\alpha))=j(\alpha)$ such that $\pi(\bar{\alpha}) \in \operatorname{acc}\left(C_{\pi(\alpha), i}\right)$. As $\pi(\bar{\alpha}) \in \operatorname{acc}(D)$, altogether, $\pi(\bar{\alpha}) \in \operatorname{acc}\left(C_{\pi(\alpha), i}\right) \cap$ $\operatorname{acc}(D)=\operatorname{acc}\left(\Phi_{D}\left(C_{\pi(\alpha), i}\right)\right)$, where the last equality is due to the definition of $\Phi_{D}$ 
and fact that $\pi(\alpha) \in \operatorname{acc}(D)$. Finally, as $\Phi_{D}\left(C_{\pi(\alpha), i}\right) \subseteq \operatorname{Im}(\pi)$, it follows that $\bar{\alpha}$ is an accumulation point of $\pi^{-1}\left[\Phi_{D}\left(C_{\pi(\alpha), i}\right)\right]=C_{\alpha, i}^{\bullet}$.

Claim 4.43.3. Let $E \subseteq \kappa$ be a club. Then there exist stationarily many $\alpha \in S$ such that, for all $C \in \mathcal{C}_{\alpha}^{\bullet}, \sup (\operatorname{nacc}(C) \cap E)=\alpha$.

Proof. Let $B$ be an arbitrary club. We need to find $\alpha \in B \cap S$ such that, for all $C \in \mathcal{C}_{\alpha}^{\bullet}, \sup (\operatorname{nacc}(C) \cap E)=\alpha$.

As $E^{\bullet}:=\{\beta \in B \cap E \mid \pi(\beta)=\beta\}$ is a club, by the choice of $D$, we may now fix $\alpha \in S$ with $\sup \left(\operatorname{nacc}\left(\Phi_{D}(C)\right) \cap E^{\bullet}\right)=\alpha$ for all $C \in \mathcal{C}_{\alpha}$. In particular, $\alpha \in \operatorname{acc}\left(E^{\bullet}\right)$, so that $\alpha \in B \cap S$ and $\pi(\alpha)=\alpha$. Consequently, $\mathcal{C}_{\alpha}^{\bullet}=\left\{\pi^{-1}\left[\Phi_{D}(C)\right] \mid C \in \mathcal{C}_{\alpha}\right\}$. Let $C \in \mathcal{C}_{\alpha}$ be arbitrary. Put $c:=\operatorname{nacc}\left(\Phi_{D}(C)\right) \cap E^{\bullet}$. Then $c$ is a subset of $\operatorname{nacc}\left(\Phi_{D}(C)\right) \cap D \cap E$, consisting of fixed-points of $\pi$. As $\sup (c)=\alpha$, it follows that $\pi^{-1}[c]=c$ is a cofinal subset of $\operatorname{nacc}\left(\pi^{-1}\left[\Phi_{D}(C)\right]\right)$. So, $c \subseteq \operatorname{nacc}\left(\pi^{-1}\left[\Phi_{D}(C)\right]\right) \cap E$ and hence the latter is cofinal in $\alpha$, as sought.

This completes the proof.

If $\overrightarrow{\mathcal{C}}=\left\langle\mathcal{C}_{\alpha} \mid \alpha<\kappa\right\rangle$ is a $\mathrm{P}^{-}\left(\kappa, \mu^{\text {ind }}, \sqsubseteq, \ldots\right)$-sequence such that $S:=\{\alpha<$ $\left.\kappa|| \mathcal{C}_{\alpha} \mid<\aleph_{0}\right\}$ is stationary, then we may find some $i<\mu$ for which $A:=$ $\left\{\alpha \in S \cap \operatorname{acc}(\kappa) \mid C_{\alpha, i}=\max \left(\mathcal{C}_{\alpha}, \subseteq\right)\right\}$ is stationary. For every $\alpha \in A, A \cap$ $\alpha \subseteq \operatorname{acc}(\alpha) \subseteq \operatorname{acc}\left(C_{\alpha, i}\right)$, and hence $\left\langle C_{\alpha, i} \mid \alpha \in A\right\rangle$ is an $\sqsubseteq$-chain converging to some club $D$ that forms a thread through $\overrightarrow{\mathcal{C}}$ (cf. Remark 2.17(3)). In particular, $\mathrm{P}^{-}\left(\kappa, \mu^{\text {ind }}, \sqsubseteq, 1,\{\kappa\}, \nu\right)$ is inconsistent for $\nu \leq \aleph_{0}$. By contrast, we have the following indexed variant of [Rin19, Theorem 3.5] 21

Theorem 4.44. Suppose $\square^{\mathrm{ind}}(\kappa, \mu)$ holds for an infinite cardinal $\mu<\kappa$.

If $\kappa=\lambda^{+}=2^{\lambda}$, then for any regular cardinal $\chi \geq \mu$ such that $\lambda^{\chi}=\lambda$, $\mathrm{P}^{-}\left(\kappa, \mu^{\text {ind }}, \sqsubseteq, 1,\left\{E_{\chi}^{\kappa}\right\}, \mu^{+}\right)$holds.

Proof. Suppose $\kappa=\lambda^{+}$and $\chi \geq \mu$ is a regular cardinal such that $\lambda^{\chi}=\lambda$. In particular, $\chi<\lambda$, so that $\mu^{+} \leq \chi^{+}<\kappa$. Now, as $\square^{\text {ind }}(\kappa, \mu)$ holds, the hypotheses of Lemma 4.43 are satisfied with $S:=E_{\chi}^{\kappa}$. Consequently, we may fix a $\mathrm{P}^{-}\left(\kappa, \mu^{\text {ind }}, \sqsubseteq, \ldots\right)$-sequence $\overrightarrow{\mathcal{C}}=\left\langle\mathcal{C}_{\alpha} \mid \alpha<\kappa\right\rangle$ with the additional property that, for every club $D \subseteq \kappa$, there exist stationarily many $\alpha \in E_{\chi}^{\kappa}$ such that $\sup (\operatorname{nacc}(C) \cap D)=\alpha$ for all $C \in \mathcal{C}_{\alpha}$.

Since $\lambda^{\chi}=\lambda$, the Engelking-Karłowicz Theorem provides a sequence $\left\langle f_{j} \mid j<\lambda\right\rangle$ of functions from $\lambda^{+}$to $\lambda$ with the property that, for every $z \in\left[\lambda^{+}\right]^{\chi}$ and every function $f: z \rightarrow \lambda$, there exists $j<\lambda$ with $f \subseteq f_{j}$. Consequently, we may fix a sequence $\left\langle f_{j} \mid j<\lambda\right\rangle$ of functions from $\lambda^{+}$to $\lambda^{+}$with the property that, for every $z \in\left[\lambda^{+}\right]^{\chi}$ and every regressive function $f: z \rightarrow \lambda^{+}$, there exists $j<\lambda$ with $f \subseteq f_{j}$.

Suppose that $2^{\lambda}=\lambda^{+}$and fix a bijection $\pi: \lambda^{+} \leftrightarrow{ }^{\lambda} \lambda^{+}$. For every $j<\lambda$, define a triangular array $\mathfrak{x}^{j}=\left\langle x_{\gamma, \beta}^{j} \mid \gamma<\beta<\kappa\right\rangle$ via:

$$
x_{\gamma, \beta}^{j}:= \begin{cases}\left\{\pi\left(f_{j}(\beta)\right)(j), \beta\right\} \cap(\gamma, \beta], & \text { if } \beta \in \operatorname{acc}(\kappa) ; \\ \{\beta\}, & \text { otherwise. }\end{cases}
$$

and then consider the corresponding acc-preserving postprocessing function $\Phi_{\mathfrak{x}^{j}}$ given by Lemma 4.9. Denote $\mathcal{C}_{\alpha}^{j}:=\left\{\Phi_{\mathfrak{x}^{j}}(C) \mid C \in \mathcal{C}_{\alpha}\right\}$, so that, for every $j<\lambda$, $\overrightarrow{\mathcal{C}^{j}}:=\left\langle\mathcal{C}_{\alpha}^{j} \mid \alpha<\kappa\right\rangle$ is yet again a $\mathrm{P}^{-}\left(\kappa, \mu^{\text {ind }}, \sqsubseteq, \ldots\right)$-sequence.

Claim 4.44.1. There is $j<\lambda$ such that $\overrightarrow{\mathcal{C}}^{j}$ witnesses $\mathrm{P}^{-}\left(\kappa, \mu^{\text {ind }}, \sqsubseteq, 1,\left\{E_{\chi}^{\kappa}\right\}, \mu^{+}, 1\right)$.

\footnotetext{
${ }^{21}$ The proofs are different, since here there is no guarantee that $\diamond^{*}\left(E_{<\lambda}^{\lambda^{+}}\right)$holds.
} 
Proof. Suppose not. Then, for every $j<\lambda$, there exists a cofinal subset $B_{j} \subseteq \kappa$ such that, for all $\alpha \in E_{\chi}^{\kappa}$, there exists $C \in \mathcal{C}_{\alpha}^{j}$ for which $\sup \left(\operatorname{nacc}(C) \cap B_{j}\right)<\alpha$. Now, let $g: \kappa \rightarrow \kappa$ denote the unique function satisfying, for every $\gamma<\kappa$ :

$$
g(\gamma)=\delta \Longleftrightarrow \bigwedge_{j<\lambda}\left(\pi(\delta)(j)=\min \left(B_{j} \backslash(\gamma+1)\right)\right) .
$$

Fix a club $E \subseteq \operatorname{acc}(\kappa)$ such that, for all $\beta \in E$ and $\gamma<\beta$,

$$
\sup \left\{g(\gamma), \min \left(B_{j} \backslash(\gamma+1)\right) \mid j<\lambda\right\}<\beta .
$$

By the choice of $\overrightarrow{\mathcal{C}}$, let us now fix $\alpha \in E_{\chi}^{\kappa}$ such that $\sup (\operatorname{nacc}(C) \cap E)=\alpha$ for all $C \in \mathcal{C}_{\alpha}$. As $\left|\mathcal{C}_{\alpha}\right| \leq \chi=\operatorname{cf}(\alpha)$, we may find a sequence $\left\langle z_{C} \mid C \in \mathcal{C}_{\alpha}\right\rangle$ of pairwise disjoint sets such that, for all $C \in \mathcal{C}_{\alpha}, z_{C}$ is a cofinal subset of nacc $(C) \cap E \backslash\{\min (C)\}$ with order-type $\chi$. Now, let $z:=\biguplus_{C \in \mathcal{C}_{\alpha}} z_{C}$. Define a function $f: z \rightarrow \lambda^{+}$as follows. For all $\beta \in z$, find the unique $C \in \mathcal{C}_{\alpha}$ such that $\beta \in z_{C}$ and then let $f(\beta):=g(\gamma)$, for $\gamma:=\sup (C \cap \beta)$. Note that as $\beta \in z \subseteq \operatorname{nacc}(C) \cap E \backslash\{\min (C)\}, \gamma<\beta$ and hence also $f(\beta)<\beta$. That is, $f$ is regressive.

Pick $j<\lambda$ such that $f \subseteq f_{j}$. By the choice of $B_{j}$, let us now pick $C^{*} \in \mathcal{C}_{\alpha}^{j}$ such that $\epsilon:=\sup \left(\operatorname{nacc}\left(C^{*}\right) \cap B_{j}\right)$ is smaller than $\alpha$. Find $C \in \mathcal{C}_{\alpha}$ such that $C^{*}=\Phi_{\mathfrak{x}^{j}}(C)$. Pick a large enough $\beta \in z_{C}$ for which $\gamma:=\sup (C \cap \beta)$ is greater than $\epsilon$. Let $\eta:=\pi\left(f_{j}(\beta)\right)(j)$. As $\gamma \in \beta \in E$, we infer that

$$
\eta=\pi\left(f_{j}(\beta)\right)(j)=\pi(g(\gamma))(j)=\min \left(B_{j} \backslash(\gamma+1)\right)<\beta,
$$

and hence $x_{\gamma, \beta}^{j}=\{\eta, \beta\}$. Recalling the definition of $\Phi_{\mathfrak{x}^{j}}$ from Lemma 4.9, we see that $\eta \in \operatorname{nacc}\left(C^{*}\right)$, contradicting the fact that $\eta \in B_{j} \backslash(\epsilon+1)$.

As $2^{\lambda}=\lambda^{+}$and $\lambda^{\aleph_{0}}=\lambda$, Gre76, Lemma 2.1] entails $\diamond\left(\lambda^{+}\right)$. So, by Fact 3.6, $\boldsymbol{\beta}(\kappa)$ holds. Then, by the proof of Theorem $4.16(1), \mathrm{P}^{-}\left(\kappa, \mu^{\text {ind }}, \sqsubseteq, 1,\left\{E_{\chi}^{\kappa}\right\}, \mu^{+}\right)$ holds, as well.

Fact 4.45 ([LL18, Theorem 3.4]). If $\square(\kappa)$ holds, then so does $\square^{\text {ind }}(\kappa, \mu)$ for every $\mu \in \operatorname{Reg}(\kappa)$.

Corollary 4.46. Suppose that $\kappa=\lambda^{+}=2^{\lambda}$ and $\square(\kappa)$ holds. For every pair $\mu \leq \chi$ of infinite regular cardinals with $\lambda^{\chi}=\lambda, \mathrm{P}^{-}\left(\kappa, \mu^{\mathrm{ind}}, \sqsubseteq, 1,\left\{E_{\chi}^{\kappa}\right\}, \mu^{+}\right)$holds.

\section{Departing From $\diamond$}

As seen in Section 2, instances of $\mathrm{P}^{-}(\kappa, \ldots)$ together with $\diamond(\kappa)$ suffice for the construction of a $\kappa$-Souslin tree. For this, in [BR17a, Definition 1.6], we defined the principle $\mathrm{P}(\kappa, \mu, \mathcal{R}, \theta, \mathcal{S}, \nu, \sigma)$ to assert both $\mathrm{P}^{-}(\kappa, \mu, \mathcal{R}, \theta, \mathcal{S}, \nu, \sigma)$ and $\diamond(\kappa)$, and then, in that paper as well as other papers in this project, we presented a gallery of constructions of $\kappa$-Souslin trees having various additional features as applications of the principle $\mathrm{P}(\kappa, \ldots)$.

The goal of this section is to present a principle $\mathrm{P}^{\bullet}(\kappa, \ldots)$ which is strong enough to still allow all of the said constructions, and weak enough to not rely on $\diamond$. Note, however, that assuming $\kappa^{<\kappa}=\kappa, \mathrm{P}^{-}\left(\kappa, \infty, \sqsubseteq_{\chi}^{*}, 1,\{\kappa\}, \kappa, \sigma\right)$ with $\sigma \geq \omega$ implies $\diamond(\kappa)$, hence, here one should really only focus on the case $\sigma=<\omega 22$ For this, we shall adopt Convention 4.18 at the outset, and will always omit the mention of $\sigma$. We shall establish the following, which is the special case $\mathcal{R}:=\sqsubseteq$ of Corollary 5.14

Corollary 5.1. For $\theta>0, \mathrm{P}_{\xi}^{\bullet}(\kappa, \mu, \sqsubseteq, \theta, \mathcal{S}, \nu)$ is equivalent to $\mathrm{P}_{\xi}^{-}(\kappa, \mu, \sqsubseteq, \theta, \mathcal{S}, \nu) \wedge$ $\left(\kappa^{<\kappa}=\kappa\right)$.

\footnotetext{
${ }^{22}$ For constructions of $\kappa$-Souslin trees that rely on instances with $\sigma=\omega$, see [BR17b].
} 
Definition 5.2. Let $\mathcal{F}(\kappa):=\bigcup_{x \in \mathcal{K}(\kappa)}{ }^{x} H_{\kappa}$ denote the collection of all functions from an element of $\mathcal{K}(\kappa)$ to $H_{\kappa}$.

For each $C \in \mathcal{F}(\kappa)$, denote $\stackrel{\circ}{C}:=\operatorname{dom}(C)$ and $\alpha_{C}:=\sup (\stackrel{\circ}{C})$.

Example 5.3. For any $C \in \mathcal{F}(\kappa), \stackrel{\circ}{C}$ is an element of $\mathcal{K}(\kappa)$. Going in the other direction, for every sequence $\left\langle A_{\beta} \mid \beta<\kappa\right\rangle$ of elements of $H_{\kappa}$, for any $x \in \mathcal{K}(\kappa)$, $C_{x}:=\left\langle A_{\beta} \mid \beta \in x\right\rangle$ is an element of $\mathcal{F}(\kappa)$.

For a binary relation $\mathcal{R}$ over $\mathcal{F}(\kappa)$, and a nonempty collection $\mathcal{S}$ of stationary subsets of $\kappa$, we shall define a principle $\mathrm{P}_{\xi}^{\bullet}(\kappa, \mu, \mathcal{R}, \theta, \mathcal{S}, \nu)$ in two stages. In the first stage, we focus on the first four parameters.

Definition 5.4. We say that $\left\langle\mathcal{C}_{\alpha} \mid \alpha<\kappa\right\rangle$ is a $\mathrm{P}_{\xi}^{\bullet}(\kappa, \mu, \mathcal{R}, \ldots)$-sequence iff, for every $\alpha \in \operatorname{acc}(\kappa)$, all of the following hold:

- $\mathcal{C}_{\alpha} \subseteq\left\{C \in \mathcal{F}(\kappa) \mid \operatorname{otp}(\stackrel{\circ}{C}) \leq \xi \& \alpha_{C}=\alpha\right\}$;

- $0<\left|\mathcal{C}_{\alpha}\right|<\mu$;

- for all $C \in \mathcal{C}_{\alpha}$ and $\bar{\alpha} \in \operatorname{acc}(\stackrel{\circ}{C})$, there exists $D \in \mathcal{C}_{\bar{\alpha}}$ with $D \mathcal{R} C$.

Convention 5.5. We shall always assume that $\mathcal{C}_{0}:=\{\emptyset\}$ and $\mathcal{C}_{\alpha+1}:=\{\{(\alpha, \emptyset)\}\}$ for all $\alpha<\kappa$. Likewise, whenever we construct a $\mathrm{P}_{\xi}^{\bullet}(\kappa, \mu, \mathcal{R}, \ldots)$-sequence $\left\langle\mathcal{D}_{\alpha}\right|$ $\alpha<\kappa\rangle$, we shall never bother to define $\mathcal{D}_{0}$ and $\mathcal{D}_{\alpha+1}$ for $\alpha<\kappa$. We also adopt Conventions 4.3, 4.6, and 4.13.

Example 5.6. The binary relations over $\mathcal{F}(\kappa)$ that fit as the parameter $\mathcal{R}$ should be understood as coherence relations. The basic example is the end-extension relation, $\sqsubseteq$, where, for $C, D \in \mathcal{F}(\kappa)$, we define $C \sqsubseteq D$ iff $C=D \uparrow \alpha_{C}$. More nuanced binary relations over $\mathcal{F}(\kappa)$ are obtained by modifying the $\sqsubseteq$ relation as follows:

- We define $C \sqsubseteq^{*} D$ iff there exists $\gamma<\alpha_{C}$ such that $C \uparrow(\stackrel{\circ}{C} \backslash \gamma) \sqsubseteq D \uparrow(\stackrel{\circ}{D} \backslash \gamma)$;

- For $\mathcal{R} \in\left\{\sqsubseteq, \sqsubseteq^{*}\right\}$, we define $C_{\chi} \mathcal{R} D$ iff $\left((C \mathcal{R} D)\right.$ or $\left.\left(\operatorname{cf}\left(\alpha_{C}\right)<\chi\right)\right)$;

- For $\mathcal{R} \in\left\{\sqsubseteq, \sqsubseteq^{*}\right\}$, we define $C \mathcal{R}_{\chi} D$ iff $((C \mathcal{R} D)$ or $(\operatorname{otp}(\stackrel{\circ}{D})<\chi$ and $\operatorname{nacc}(\stackrel{D}{D})$ consists only of successor ordinals));

- For any binary relation $\mathcal{R}$ over $\mathcal{F}(\kappa)$ and any class $\Omega \subseteq$ ORD, we define $C{ }^{\Omega} \mathcal{R} D$ iff $\left((C \mathcal{R} D)\right.$ and $\left.\left(\alpha_{C} \notin \Omega\right)\right)$.

The principle $\mathrm{P}^{-}(\kappa, \ldots)$ of the previous section (Definition 4.10) dealt with hitting of arbitrary cofinal subsets of $\kappa$. The new principle $\mathrm{P}^{\bullet}(\kappa, \ldots)$ focuses on hitting sets of the sort arising by Proposition 2.12 and by the following strengthening of Fact 2.13 .

Fact 5.7 (BR17a, Lemma 2.2]). $\diamond(\kappa)$ is equivalent to the existence of a sequence $\left\langle A_{\beta} \mid \beta<\kappa\right\rangle$ of elements of $H_{\kappa}$ and a partition $\left\langle B_{\iota} \mid \iota<\kappa\right\rangle$ of $\kappa$, such that, for every subset $\Omega \subseteq H_{\kappa}$, every parameter $p \in H_{\kappa^{+}}$, and every $\iota<\kappa$, the following set is cofinal in $\kappa$ :

$$
B_{\iota}(\Omega, p):=\left\{\beta \in B_{\iota} \mid \exists \mathcal{M} \prec H_{\kappa^{+}}\left(\mathcal{M} \cap \Omega=A_{\beta} \& p \in \mathcal{M} \& \mathcal{M} \cap \kappa=\beta\right)\right\} .
$$

As the reader by now probably expects, the cofinal sets considered by $\mathrm{P}^{\bullet}(\kappa, \ldots)$ take the following form.

Definition 5.8. Given $B \subseteq \kappa, \Omega \subseteq H_{\kappa}, p \in H_{\kappa^{+}}$and $C \in \mathcal{F}(\kappa)$, we let $B(\Omega, p, C)$

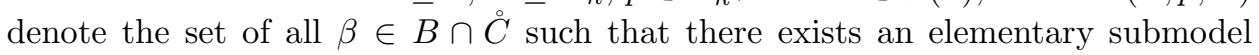
$\mathcal{M} \prec H_{\kappa^{+}}$satisfying:

- $p \in \mathcal{M}$;

- $\mathcal{M} \cap \kappa=\beta$;

- $\mathcal{M} \cap \Omega=C(\beta)$.

We now arrive at the second stage of the definition of this principle. 
Definition 5.9. $\mathrm{P}_{\xi}^{\bullet}(\kappa, \mu, \mathcal{R}, \theta, \mathcal{S}, \nu)$ asserts the existence of a $\mathrm{P}_{\xi}^{\bullet}(\kappa, \mu, \mathcal{R}, \ldots)$-sequence $\left\langle\mathcal{C}_{\alpha} \mid \alpha<\kappa\right\rangle$ and a partition $\left\langle B_{\iota} \mid \iota<\kappa\right\rangle$ of $\kappa$, satisfying the following.

For every sequence $\left\langle\left(\Omega_{i}, p_{i}, \iota_{i}\right) \mid i<\theta\right\rangle$ of elements of $\mathcal{P}\left(H_{\kappa}\right) \times H_{\kappa^{+}} \times \kappa$, every $S \in \mathcal{S}$, and every $n<\omega$, there exist stationarily many $\alpha \in S$ such that:

(1) $\left|\mathcal{C}_{\alpha}\right|<\nu$; and

(2) for all $C \in \mathcal{C}_{\alpha}$ and $i<\min \{\alpha, \theta\}$,

$$
\sup \left\{\gamma \in \stackrel{\circ}{C} \mid \operatorname{succ}_{n}(\stackrel{\circ}{C} \backslash \gamma) \subseteq B_{\iota_{i}}\left(\Omega_{i}, p_{i}, C\right)\right\}=\alpha .
$$

Before we prove the main result of this section, let us point out that $\mathrm{P}^{\bullet}(\kappa, \ldots)$ is indeed a consequence of $\mathrm{P}(\kappa, \ldots)$.

Proposition 5.10. Suppose that $\mathrm{P}_{\xi}^{-}(\kappa, \mu, \mathcal{R}, \theta, \mathcal{S}, \nu)+\diamond(\kappa)$ holds, with $\mathcal{R}$ from Example 5.6. Then so does $\mathrm{P}_{\xi}^{\bullet}(\kappa, \mu, \mathcal{R}, \theta, \mathcal{S}, \nu)$.

Proof. Recalling Convention 4.18, fix a sequence $\left\langle\mathcal{C}_{\alpha} \mid \alpha<\kappa\right\rangle$ witnessing $\mathrm{P}_{\xi}^{-}(\kappa, \mu, \mathcal{R}$, $\theta, \mathcal{S}, \nu,<\omega)$. As $\diamond(\kappa)$ holds, let $\left\langle A_{\beta} \mid \beta<\kappa\right\rangle$ and $\left\langle B_{\iota} \mid \iota<\kappa\right\rangle$ be given by Fact 5.7 .

For every $x \in \mathcal{K}(\kappa)$, let $D_{x}:=\left\langle A_{\beta} \mid \beta \in x\right\rangle$. Then, for every $\alpha \in \operatorname{acc}(\kappa)$, let $\mathcal{D}_{\alpha}:=\left\{D_{x} \mid x \in \mathcal{C}_{\alpha}\right\}$. Evidently, $\left\langle\mathcal{D}_{\alpha} \mid \alpha<\kappa\right\rangle$ and $\left\langle B_{\iota} \mid \iota<\kappa\right\rangle$ together witness $\mathrm{P}_{\xi}^{\bullet}(\kappa, \mu, \mathcal{R}, \theta, \mathcal{S}, \nu)$.

Proposition 5.11. If $\mathrm{P}_{\xi}^{\bullet}(\kappa, \mu, \mathcal{R}, \theta, \mathcal{S}, \nu)$ holds with $\theta>0$, then $\kappa^{<\kappa}=\kappa$.

Proof. Let $\overrightarrow{\mathcal{C}}=\left\langle\mathcal{C}_{\alpha} \mid \alpha<\kappa\right\rangle$ be a sequence that, together with some partition $\left\langle B_{\iota}\right|$ $\iota<\kappa\rangle$ of $\kappa$, witnesses $\mathrm{P}_{\xi}^{\bullet}(\kappa, \mu, \mathcal{R}, \theta, \mathcal{S}, \nu)$ with $\theta>0$.

Claim 5.11.1. $H_{\kappa}=\bigcup_{\alpha \in \operatorname{acc}(\kappa)} \bigcup_{C \in \mathcal{C}_{\alpha}} \operatorname{Im}(C)$.

Proof. We focus on the nontrivial inclusion. Let $A \in H_{\kappa}$. Set $\Omega_{0}:=p_{0}:=A$, $\iota_{0}:=0$, and $n:=1$. Then by the hypothesis on the sequence $\overrightarrow{\mathcal{C}}$, we can choose some $\alpha \in \operatorname{acc}(\kappa)$ such that for all $C \in \mathcal{C}_{\alpha}$, Equation (체 $)$ holds with $i:=0$. Choose some $C \in \mathcal{C}_{\alpha}$ and some $\beta \in \operatorname{nacc}(\stackrel{\circ}{C}) \cap B_{0}(A, A, C)$. Then we can fix an elementary submodel $\mathcal{M} \prec H_{\kappa^{+}}$such that $A \in \mathcal{M}, \mathcal{M} \cap \kappa=\beta$, and $\mathcal{M} \cap A=C(\beta)$. But $|A|<\kappa$ and $|A| \in \mathcal{M}$ by elementarity, so that $|A| \in \mathcal{M} \cap \kappa=\beta$, and we infer that $A \subseteq \mathcal{M}$. Thus, $A=A \cap \mathcal{M}=C(\beta) \in \operatorname{Im}(C)$.

In particular, $\left|H_{\kappa}\right|=\kappa$.

Lemma 5.12. Suppose $\mathcal{R}$ is taken from Example 5.6. Then $\mathrm{P}_{\xi}^{\bullet}(\kappa, \mu, \mathcal{R}, \theta, \mathcal{S}, \nu)$ implies $\mathrm{P}_{\xi}^{-}(\kappa, \mu, \mathcal{R}, \theta, \mathcal{S}, \nu)$.

Proof. For every $C \in \mathcal{F}(\kappa)$, let $x_{C}:=\operatorname{Im}\left(g_{C}\right)$, where the function $g_{C}: \stackrel{\circ}{C} \rightarrow \alpha_{C}$ is defined by setting

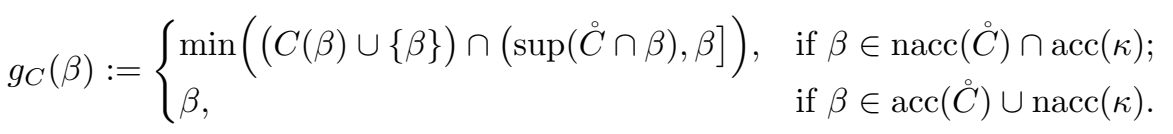

Claim 5.12.1. For all $C, C^{\prime} \in \mathcal{F}(\kappa)$ :

(1) $x_{C}$ is a club in $\alpha_{C}$ with $\operatorname{acc}\left(x_{C}\right)=\operatorname{acc}(\stackrel{\circ}{C})$.

(2) If $C \mathcal{R} C^{\prime}$ then $x_{C} \mathcal{R} x_{C^{\prime}}$.

Proof. Left to the reader (cf. Lemma 4.9).

Fix a sequence $\overrightarrow{\mathcal{C}}=\left\langle\mathcal{C}_{\alpha} \mid \alpha<\kappa\right\rangle$ and a partition $\vec{B}=\left\langle B_{\iota} \mid \iota<\kappa\right\rangle$ of $\kappa$ together witnessing $\mathrm{P}_{\xi}^{\bullet}(\kappa, \mu, \mathcal{R}, \theta, \mathcal{S}, \nu)$. Then, for every $\alpha \in \operatorname{acc}(\kappa)$, let $\mathcal{D}_{\alpha}:=\left\{x_{C} \mid C \in \mathcal{C}_{\alpha}\right\}$. It follows from Claim 5.12.1 that $\overrightarrow{\mathcal{D}}:=\left\langle\mathcal{D}_{\alpha} \mid \alpha<\kappa\right\rangle$ is a $\mathrm{P}_{\xi}^{-}(\kappa, \mu, \mathcal{R}, \ldots)$-sequence. To show that $\overrightarrow{\mathcal{D}}$ witnesses $\mathrm{P}_{\xi}^{-}(\kappa, \mu, \mathcal{R}, \theta, \mathcal{S}, \nu)$, we shall now verify that it satisfies the hitting feature of Definition 4.10, recalling Convention 4.18, 
Claim 5.12.2. Suppose $S \in \mathcal{S},\left\langle A_{i} \mid i<\theta\right\rangle$ is a sequence of cofinal subsets of $\kappa$, and $n<\omega$. Then there exist stationarily many $\alpha \in S$ such that $\left|\mathcal{D}_{\alpha}\right|<\nu$ and, for all $x \in \mathcal{D}_{\alpha}$ and all $i<\min \{\alpha, \theta\}, \sup \left\{\delta \in x \mid \operatorname{succ}_{n}(x \backslash \delta) \subseteq A_{i}\right\}=\alpha$.

Proof. For every $i<\theta$, let $\Omega_{i}:=p_{i}:=A_{i}$ and $\iota_{i}:=0$. By the choice of $\overrightarrow{\mathcal{C}}$ and $\vec{B}$, there are stationarily many $\alpha \in S \cap \operatorname{acc}(\kappa)$ such that $\left|\mathcal{C}_{\alpha}\right|<\nu$ and, for all $C \in \mathcal{C}_{\alpha}$ and all $i<\min \{\alpha, \theta\}, \sup \left\{\gamma \in \stackrel{\circ}{C} \mid \operatorname{succ}_{n+1}(\stackrel{\circ}{C} \backslash \gamma) \subseteq B_{0}\left(A_{i}, A_{i}, C\right)\right\}=\alpha$. Consider any such $\alpha$. Clearly, $\left|\mathcal{D}_{\alpha}\right| \leq\left|\mathcal{C}_{\alpha}\right|<\nu$. Now, consider any given $x \in \mathcal{D}_{\alpha}$, $i<\min \{\alpha, \theta\}$, and $\varepsilon<\alpha$. By definition of $\mathcal{D}_{\alpha}$, we can fix some $C \in \mathcal{C}_{\alpha}$ such that $x=x_{C}$. By our choice of $\alpha$, we can fix $\gamma \in \stackrel{\circ}{C}$ with $\varepsilon<\gamma<\alpha$ such that $\operatorname{succ}_{n+1}(\stackrel{\circ}{C} \backslash \gamma) \subseteq B_{0}\left(A_{i}, A_{i}, C\right)$.

Consider each $j<n+1$. Set $\beta_{j}:=(\stackrel{\circ}{C} \backslash \gamma)(j+1)$. Then $\beta_{j} \in B_{0}\left(A_{i}, A_{i}, C\right)$, meaning that we can fix an elementary submodel $\mathcal{M}_{j} \prec H_{\kappa^{+}}$satisfying $A_{i} \in \mathcal{M}_{j}$, $\mathcal{M}_{j} \cap \kappa=\beta_{j}$, and $\mathcal{M}_{j} \cap A_{i}=C\left(\beta_{j}\right)$. As $A_{i}$ is a cofinal subset of $\kappa$, it follows by elementarity that $\mathcal{M}_{j} \models$ " $A_{i}$ is a cofinal subset of $\kappa$ ", so that in fact $C\left(\beta_{j}\right)$ is a cofinal subset of $\beta_{j}$. Of course, $\beta_{j} \in \operatorname{acc}(\kappa)$. Thus, as $\beta_{j} \in \operatorname{nacc}(\stackrel{\circ}{C})$, we infer from the definition of $g_{C}$ that $g_{C}\left(\beta_{j}\right) \in C\left(\beta_{j}\right) \subseteq A_{i}$.

It is clear that $\gamma, \beta_{0}, \beta_{1}, \ldots, \beta_{n}$ are $\mathrm{n}+2$ consecutive points of $\stackrel{\circ}{C}$. Altogether, we infer from the definition of $x_{C}$ that $g_{C}\left(\beta_{0}\right), g_{C}\left(\beta_{1}\right), \ldots, g_{C}\left(\beta_{n}\right)$ are $\mathrm{n}+1$ consecutive points of $x_{C}$, all above $\gamma$. Letting $\delta:=g_{C}\left(\beta_{0}\right)$, we obtain $\varepsilon<\gamma<\delta, \delta \in x$, and $\operatorname{succ}_{n}(x \backslash \delta) \subseteq A_{i}$, as sought.

It follows that $\overrightarrow{\mathcal{D}}$ is as sought.

We now improve Proposition 5.10 in two ways. First, we reduce $\diamond(\kappa)$ down to $\kappa^{<\kappa}=\kappa$. Second, and more surprisingly, we let our reader choose the partition of $\kappa$.

Theorem 5.13. Suppose that $\kappa^{<\kappa}=\kappa$ and $\mathrm{P}_{\xi}^{-}(\kappa, \mu, \mathcal{R}, \theta, \mathcal{S}, \nu)$ holds with $\mathcal{R}$ from Example 5.6. Let $\vec{B}=\left\langle B_{\iota} \mid \iota<\kappa\right\rangle$ be a given partition of $\kappa$ into stationary sets. Then there exists a sequence $\left\langle\mathcal{C}_{\alpha} \mid \alpha<\kappa\right\rangle$ that, together with $\vec{B}$, witnesses $\mathrm{P}_{\xi}^{\bullet}(\kappa, \mu, \mathcal{R}, \theta, \mathcal{S}, \nu)$.

Proof. Using $\kappa^{<\kappa}=\kappa$, let $\triangleleft$ be some well-ordering of $H_{\kappa}$ of order-type $\kappa$. Also, fix a sequence $\vec{A}=\left\langle A_{\gamma} \mid \gamma<\kappa\right\rangle$ of elements of $H_{\kappa}$ such that:

- for each $\gamma \in \operatorname{nacc}(\kappa), A_{\gamma}=\emptyset$, and

- for all $\iota<\kappa$ and $A \in H_{\kappa},\left\{\gamma \in B_{\iota} \mid A_{\gamma}=A\right\}$ is stationary in $\kappa$.

For every $\gamma<\kappa$, let $\beta_{\gamma}:=\operatorname{otp}\left(A_{\gamma}, \triangleleft\right)$.

Claim 5.13.1. Let $\iota<\kappa, \Omega \in\left[H_{\kappa}\right]^{\kappa}$ and $p \in H_{\kappa^{+}}$. There exists $G \subseteq \kappa$ such that $\left\{\beta_{\gamma} \mid \gamma \in G\right\}$ is cofinal in $\kappa$, and, for every $\gamma \in G$, there exists an elementary submodel $\mathcal{M} \prec H_{\kappa^{+}}$such that:

$$
p \in \mathcal{M}, \quad \mathcal{M} \cap \kappa=\beta_{\gamma}, \quad \mathcal{M} \cap \Omega=A_{\gamma}, \text { and } \beta_{\gamma} \in B_{\iota} \cap \gamma .
$$

Proof. Let $\epsilon<\kappa$ be arbitrary; we shall exhibit the existence of an ordinal $\gamma<\kappa$ with $\beta_{\gamma}>\epsilon$ satisfying all of the four requirements.

As $B_{\iota}$ is stationary, we may pick $\mathcal{M} \prec H_{\kappa^{+}}$with $p, \triangleleft, \Omega \in \mathcal{M}$ such that $\mathcal{M} \cap \kappa \in$ $B_{\iota} \backslash(\epsilon+1)$. Denote $\beta:=\mathcal{M} \cap \kappa$. As $\triangleleft \in \mathcal{M}$, we infer that $\left|\mathcal{M} \cap H_{\kappa}\right|=|\beta|<\kappa$, so that $\mathcal{M} \cap \Omega \in H_{\kappa}$. Thus, by the choice of $\vec{A}$, let us fix $\gamma>\beta$ such that $\mathcal{M} \cap \Omega=A_{\gamma}$. Finally, as $H_{\kappa^{+}} \models \operatorname{otp}(\Omega, \triangleleft)=\kappa$, we infer that $\beta_{\gamma}=\operatorname{otp}\left(A_{\gamma}, \triangleleft\right)=\operatorname{otp}(\mathcal{M} \cap \Omega, \triangleleft)=$ $\kappa^{\mathcal{M}}=\beta$.

Define $c:[\kappa]^{2} \rightarrow \kappa$ by letting, for all $\delta<\gamma<\kappa$,

$$
c(\delta, \gamma):= \begin{cases}\beta_{\gamma}, & \text { if } \beta_{\gamma} \in(\delta, \gamma] \\ \gamma, & \text { otherwise. }\end{cases}
$$


Let $x \in \mathcal{K}(\kappa)$ be arbitrary. We define an element $C_{x}$ of $\mathcal{F}(\kappa)$, as follows:

- $\operatorname{dom}\left(C_{x}\right):=\operatorname{acc}(x) \cup\{c(\sup (x \cap \gamma), \gamma) \mid \gamma \in \operatorname{nacc}(x) \& \gamma \neq \min (x)\}$;

- for all $\beta \in \operatorname{dom}\left(C_{x}\right)$, we let $C_{x}(\beta):=A_{\min (x \backslash \beta)}$.

Claim 5.13.2. For all $x, y \in \mathcal{K}(\kappa)$ :

(1) $\operatorname{dom}\left(C_{x}\right)$ is a club in $\sup (x)$ with $\operatorname{acc}\left(\operatorname{dom}\left(C_{x}\right)\right)=\operatorname{acc}(x)$;

(2) if $x \mathcal{R} y$, then $C_{x} \mathcal{R} C_{y}$.

Proof. Left to the reader.

Recalling Convention 4.18, fix a sequence $\overrightarrow{\mathcal{D}}=\left\langle\mathcal{D}_{\alpha} \mid \alpha<\kappa\right\rangle$ witnessing $\mathrm{P}_{\xi}^{-}(\kappa, \mu$, $\mathcal{R}, \theta, \mathcal{S}, \nu,<\omega)$. Then, for every $\alpha \in \operatorname{acc}(\kappa)$, let $\mathcal{C}_{\alpha}:=\left\{C_{x} \mid x \in \mathcal{D}_{\alpha}\right\}$.

Claim 5.13.3. Suppose that $S \in \mathcal{S}$ and $\left\langle\left(\Omega_{i}, p_{i}, \iota_{i}\right) \mid i<\theta\right\rangle$ is a sequence of elements of $\mathcal{P}\left(H_{\kappa}\right) \times H_{\kappa^{+}} \times \kappa$, and $n$ is a positive integer. Then there exist stationarily many $\alpha \in S$ such that $\left|\mathcal{C}_{\alpha}\right|<\nu$, and, for every $C \in \mathcal{C}_{\alpha}$ and $i<\min \{\alpha, \theta\}$,

$$
\sup \left\{\beta \in \stackrel{\circ}{C} \mid \operatorname{succ}_{n}(\stackrel{\circ}{C} \backslash \beta) \subseteq B_{\iota_{i}}\left(\Omega_{i}, p_{i}, C\right)\right\}=\alpha .
$$

Proof. Let $i<\theta$ be arbitrary.

- If $\left|\Omega_{i}\right|=\kappa$, then, by Claim 5.13.1, we may fix a cofinal subset $G_{i} \subseteq \kappa$ such that, for every $\gamma \in G_{i}$, there exists an elementary submodel $\mathcal{M} \prec H_{\kappa^{+}}$, such that:

$p_{i} \in \mathcal{M}, \quad \mathcal{M} \cap \kappa=\beta_{\gamma}, \quad \mathcal{M} \cap \Omega_{i}=A_{\gamma}, \quad \beta_{\gamma} \in B_{\iota_{i}} \cap \gamma$, and $\quad G_{i} \cap \gamma=G_{i} \cap \beta_{\gamma}$.

- If $\left|\Omega_{i}\right|<\kappa$, then as $\left\{\gamma \in B_{\iota_{i}} \mid A_{\gamma}=\Omega_{i}\right\}$ is stationary, we may fix a cofinal subset $G_{i} \subseteq \kappa$ such that, for every $\gamma \in G_{i}$, there exists an elementary submodel $\mathcal{M} \prec H_{\kappa^{+}}$, such that:

$$
p_{i}, \triangleleft, \Omega_{i} \in \mathcal{M}, \quad \mathcal{M} \cap \kappa=\gamma, \quad \Omega_{i}=A_{\gamma}, \text { and } \gamma \in B_{\iota_{i}} .
$$

Next, fix a club $E \subseteq \kappa$ with the property that, for every $\alpha \in E$ and $i<\min \{\alpha, \theta\}$, if $\left|\Omega_{i}\right|<\kappa$, then $\operatorname{otp}\left(\Omega_{i}, \triangleleft\right)<\alpha$. By the choice of $\overrightarrow{\mathcal{D}}$, we may find a stationary $S^{\prime} \subseteq S \cap E$ such that, for every $\alpha \in S^{\prime},\left|\mathcal{D}_{\alpha}\right|<\nu$, and, for all $x \in \mathcal{D}_{\alpha}$ and $i<\min \{\alpha, \theta\}$ :

$$
\sup \left\{\gamma \in x \mid \operatorname{succ}_{n+1}(x \backslash \gamma) \subseteq G_{i}\right\}=\alpha .
$$

Let $\alpha \in S^{\prime}$ be arbitrary. Clearly, $\left|\mathcal{C}_{\alpha}\right| \leq\left|\mathcal{D}_{\alpha}\right|<\nu$. Fix $x \in \mathcal{D}_{\alpha}$ and $i<\min \{\alpha, \theta\}$; we need to prove that for every $\epsilon<\alpha$, there exists $\beta \in \stackrel{\circ}{C}_{x} \backslash \epsilon$ with

$$
\operatorname{succ}_{n}\left(\stackrel{\circ}{C}_{x} \backslash \beta\right) \subseteq B_{\iota_{i}}\left(\Omega_{i}, p_{i}, C_{x}\right) .
$$

As $\alpha \in S^{\prime}$, let us fix a large enough $\gamma \in x \backslash \epsilon \operatorname{such}$ that $\operatorname{succ}_{n+1}(x \backslash \gamma) \subseteq G_{i}$. If $\left|\Omega_{i}\right|<\kappa$, then we also require that $\operatorname{otp}\left(\Omega_{i}, \triangleleft\right) \leq \gamma$, which is possible since $\alpha \in E$.

Let $\left\{\gamma_{j} \mid j<n+1\right\}$ denote the increasing enumeration of $\operatorname{succ}_{n+1}(x \backslash \gamma)$. Set $\beta:=c\left(\gamma, \gamma_{0}\right)$, so that $\beta \in \stackrel{\circ}{C}_{x} \backslash \epsilon$ and $\operatorname{succ}_{n}\left(\dot{C}_{x} \backslash \beta\right)=\left\{c\left(\gamma_{j}, \gamma_{j+1}\right) \mid j<n\right\}$.

Fix an arbitrary $j<n$, and we shall show that $c\left(\gamma_{j}, \gamma_{j+1}\right) \in B_{\iota_{i}}\left(\Omega_{i}, p_{i}, C_{x}\right)$.

- If $\left|\Omega_{i}\right|=\kappa$, then as $\gamma_{j+1} \in G_{i}$, pick $\mathcal{M} \prec H_{\kappa^{+}}$such that:

- $p_{i} \in \mathcal{M}$;

- $\mathcal{M} \cap \kappa=\beta_{\gamma_{j+1}}$

- $\mathcal{M} \cap \Omega_{i}=A_{\gamma_{j+1}}$;

- $\beta_{\gamma_{j+1}} \in B_{\iota_{i}} \cap \gamma_{j+1}$;

- $G_{i} \cap \gamma_{j+1}=G_{i} \cap \beta_{\gamma_{j+1}}$.

By the last two bullets, $\gamma_{j}<\beta_{\gamma_{j+1}}<\gamma_{j+1}$, so that $c\left(\gamma_{j}, \gamma_{j+1}\right)=\beta_{\gamma_{j+1}}$. Furthermore, $\beta_{\gamma_{j+1}}$ is the unique element of the interval $\dot{C}_{x} \cap\left(\gamma_{j}, \gamma_{j+1}\right)$, so that $C_{x}\left(\beta_{\gamma_{j+1}}\right)=A_{\min \left(x \backslash \beta_{\gamma_{j+1}}\right)}=A_{\gamma_{j+1}}=\mathcal{M} \cap \Omega_{i}$. Altogether, $\mathcal{M}$ witnesses that $c\left(\gamma_{j}, \gamma_{j+1}\right) \in B_{\iota_{i}}\left(\Omega_{i}, p_{i}, C_{x}\right)$.

- If $\left|\Omega_{i}\right|<\kappa$, then as $\gamma_{j+1} \in G_{i}$, pick $\mathcal{M} \prec H_{\kappa^{+}}$such that:

- $p_{i}, \triangleleft, \Omega_{i} \in \mathcal{M}$; 
- $\mathcal{M} \cap \kappa=\gamma_{j+1}$;

- $\Omega_{i}=A_{\gamma_{j+1}}$

- $\gamma_{j+1} \in B_{\iota_{i}}$.

As $\beta_{\gamma_{j+1}}=\operatorname{otp}\left(A_{\gamma_{j+1}}, \triangleleft\right)=\operatorname{otp}\left(\Omega_{i}, \triangleleft\right) \leq \gamma<\gamma_{j}<\gamma_{j+1}$, we infer that $\mathcal{M} \cap \Omega_{i}=$ $\Omega_{i}=A_{\gamma_{j+1}}$ and $c\left(\gamma_{j}, \gamma_{j+1}\right)=\gamma_{j+1}$. In particular, $C_{x}\left(\gamma_{j+1}\right)=A_{\min \left(x \backslash \gamma_{j+1}\right)}=$ $A_{\gamma_{j+1}}=\mathcal{M} \cap \Omega_{i}$. Altogether, $\mathcal{M}$ witnesses that $c\left(\gamma_{j}, \gamma_{j+1}\right) \in B_{\iota_{i}}\left(\Omega_{i}, p_{i}, C_{x}\right)$.

It follows that $\left\langle\mathcal{C}_{\alpha} \mid \alpha<\kappa\right\rangle$ is as sought.

Putting the last three results together, we obtain:

Corollary 5.14. For $\theta>0$, and $\mathcal{R}$ from Example 5.6, the following are equivalent:

(1) $\mathrm{P}_{\xi}^{-}(\kappa, \mu, \mathcal{R}, \theta, \mathcal{S}, \nu) \wedge\left(\kappa^{<\kappa}=\kappa\right)$;

(2) $\mathrm{P}_{\xi}^{\bullet}(\kappa, \mu, \mathcal{R}, \theta, \mathcal{S}, \nu)$;

(3) For every partition $\vec{B}=\left\langle B_{\iota} \mid \iota<\kappa\right\rangle$ of $\kappa$ into stationary sets, there exists a sequence $\overrightarrow{\mathcal{C}}$ that, together with $\vec{B}$, witnesses $\mathrm{P}_{\xi}^{\bullet}(\kappa, \mu, \mathcal{R}, \theta, \mathcal{S}, \nu)$.

The following combines Definition 4.42 with Definition 5.9 .

Definition 5.15. $\mathrm{P}_{\xi}^{\bullet}\left(\kappa, \mu^{\text {ind }}, \sqsubseteq, \theta, \mathcal{S}, \nu\right)$ asserts the existence of sequences $\overrightarrow{\mathcal{C}}=\left\langle\mathcal{C}_{\alpha}\right|$ $\alpha<\kappa\rangle, \vec{B}=\left\langle B_{\iota} \mid \iota<\kappa\right\rangle$, and $\langle i(\alpha) \mid \alpha<\kappa\rangle$ such that $\overrightarrow{\mathcal{C}}$ and $\vec{B}$ together witness $\mathrm{P}_{\xi}^{\bullet}\left(\kappa, \mu^{+}, \sqsubseteq, \theta, \mathcal{S}, \nu\right)$, and for every $\alpha \in \operatorname{acc}(\kappa)$, all of the following hold:

- there exists a canonical enumeration $\left\langle C_{\alpha, i} \mid i(\alpha) \leq i<\mu\right\rangle$ of $\mathcal{C}_{\alpha}$;

- for all $i \in[i(\alpha), \mu)$ and $\bar{\alpha} \in \operatorname{acc}\left(\stackrel{\circ}{C}_{\alpha, i}\right), i \geq i(\bar{\alpha})$ and $C_{\bar{\alpha}, i} \sqsubseteq C_{\alpha, i}$;

- $\left\langle\operatorname{acc}\left(\stackrel{\circ}{C}_{\alpha, i}\right) \mid i(\alpha) \leq i<\mu\right\rangle$ is $\subseteq$-increasing with $\operatorname{acc}(\alpha)=\bigcup_{i \in[i(\alpha), \mu)} \operatorname{acc}\left(\stackrel{\circ}{C}_{\alpha, i}\right)$.

The proofs in this section make clear that the following holds as well.

Corollary 5.16. Suppose that $\theta>0$ and $\mu<\kappa$. Then $\mathrm{P}_{\xi}^{\bullet}\left(\kappa, \mu^{\mathrm{ind}}, \sqsubseteq, \theta, \mathcal{S}, \nu\right)$ is equivalent to $\mathrm{P}_{\xi}^{-}\left(\kappa, \mu^{\text {ind }}, \sqsubseteq, \theta, \mathcal{S}, \nu\right) \wedge\left(\kappa^{<\kappa}=\kappa\right)$.

\section{Tree constructions}

In this section, we present various constructions from instances of the proxy principle $\mathrm{P}^{\bullet}(\kappa, \ldots)$. The next table summarizes the kind of $\kappa$-Souslin trees we obtain from the instance $\mathrm{P}^{\bullet}\left(\kappa, \mu, \mathcal{R}, \theta,\left\{E_{>\partial}^{\kappa}\right\}, \nu\right)$. Of course, for the $\chi$-complete trees, we must also assume that $\kappa$ is $(<\chi)$-closed, or we can simply assume that $\chi=\aleph_{0}$.

\begin{tabular}{|c|c|c|c|c|c|c|}
\hline Theorem & $\mu$ & $\mathcal{R}$ & $\theta$ & $\partial$ & $\nu$ & Type of $\kappa$-Souslin tree \\
\hline 6.8 & 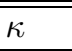 & $\chi \sqsubseteq^{* *}$ & 1 & $\chi$ & $\kappa$ & $\chi$-complete \\
\hline 6.11 & $\mu^{\text {ind }}$ & $\sqsubseteq$ & 1 & $\chi$ & $\kappa$ & $\chi$-complete with a $\mu$-ascent path \\
\hline 6.14 & $\kappa$ & $\chi \sqsubseteq^{*}$ & 1 & $\max \{\chi, \lambda\}$ & 2 & $\begin{array}{l}\chi \text {-complete, } \\
\text { with no ascending path of width }<\lambda\end{array}$ \\
\hline 6.17 & $\kappa$ & $\chi \sqsubseteq$ & 1 & $\max \{\chi, \lambda\}$ & 2 & $\begin{array}{l}\bar{\chi} \text {-complete, rigid, } \\
\text { with no ascending path of width }<\lambda\end{array}$ \\
\hline 6.27 & $\kappa$ & $\chi \sqsubseteq$ & $\kappa$ & $\chi$ & 2 & $\chi$-complete, $\chi$-free \\
\hline 6.33 & $\kappa$ & $\chi \coprod^{*}$ & $\kappa$ & $\chi$ & $\kappa$ & $\chi$-complete, uniformly homogeneous \\
\hline 6.35 & 2 & $\sqsubseteq$ & $\kappa$ & 0 & 2 & slim, uniformly coherent \\
\hline
\end{tabular}

FIGURE 1. Relationship between the vector of parameters and the characteristics of trees obtained.

By SZ99, every uniformly coherent $\omega_{1}$-Souslin tree is the product of two free $\omega_{1}$-Souslin trees. In addition, it is not hard to see that for any cardinal $\lambda$, any 
$\lambda$-free $\lambda^{+}$-Souslin tree is specializable. Finally, by a result of Baumgartner that we mentioned in the introduction, there consistently exist $\omega_{2}$-Souslin trees which are not specializable. This suggests that uniformly coherent $>$ free $>$ specializable $>$ plain, and this claim is in fact supported by the results summarized in the above table. Indeed, for a uniformly coherent $\kappa$-Souslin tree, we assume $\mu=\nu=2$ and $\theta=\kappa$; for a free $\kappa$-Souslin tree, we (allow $\mu=\kappa$ but) assume $\nu=2$ and $\theta=\kappa$; for a $\kappa$-Souslin tree omitting a narrow ascending path (which is a generalization of specializable), we assume $\nu=2$ and $\theta=1$, whereas, for a plain $\kappa$-Souslin tree, $\nu=\kappa$ and $\theta=1$ will do.

The next list demonstrates well the utility of the proxy principle as a device that provides a disconnection between the tree constructions and the study of the combinatorial hypotheses 23

Theorem 6.1. (1) Assuming $\lambda=\operatorname{cf}(\lambda) \geq \aleph_{0}, \diamond\left(E_{\lambda}^{\lambda^{+}}\right)$entails $\mathrm{P}_{\lambda}^{\bullet}\left(\lambda^{+}, 2,{ }_{\lambda} \sqsubseteq\right.$, $\left.\lambda^{+},\left\{E_{\lambda}^{\lambda^{+}}\right\}, 2\right)$. In particular, $\diamond\left(\omega_{1}\right)$ entails $\mathrm{P}_{\omega}^{\bullet}\left(\omega_{1}, 2, \sqsubseteq, \omega_{1},\left\{\omega_{1}\right\}, 2\right)$.

(2) Assuming $\lambda=\operatorname{cf}(\lambda) \geq \aleph_{0}, \diamond^{*}\left(E_{\lambda}^{\lambda^{+}}\right)$entails $\mathrm{P}_{\lambda}^{\bullet}\left(\lambda^{+}, 2,{ }_{\lambda} \sqsubseteq, \lambda^{+}, \mathrm{NS}_{\lambda^{+}}^{+}\left\lceil E_{\lambda^{\lambda^{+}}}, 2\right)\right.$.

(3) Assuming $\lambda \geq \aleph_{0}, \nabla_{\lambda}$ entails $\mathrm{P}_{\lambda}^{\bullet}\left(\lambda^{+}, 2, \sqsubseteq, \lambda^{+},\left\{\lambda^{+}\right\}, 2\right)$.

(4) Assuming $\lambda \geq \aleph_{1}, \square_{\lambda}+\mathrm{CH}_{\lambda}$ entails $\mathrm{P}_{\lambda}^{\bullet}\left(\lambda^{+}, 2\right.$, $\left.,<\lambda,\left\{E_{\chi}^{\lambda^{+}}\right\}, 2\right)$ for every $\chi \in \operatorname{Reg}(\lambda)$, as well as $\mathrm{P}^{\bullet}\left(\lambda^{+}, 2, \sqsubseteq^{*}, 1,\left\{E_{\lambda}^{\lambda^{+}}\right\}, 2\right)$ for $\lambda$ regular.

(5) Assuming $\lambda \geq \beth_{\omega}, \square\left(\lambda^{+}\right)+\mathrm{CH}_{\lambda}$ entails $\mathrm{P}^{\bullet}\left(\lambda^{+}, 2, \sqsubseteq, 1,\left\{\lambda^{+}\right\}, 2\right)$, as well as $\mathrm{P}^{\bullet}\left(\lambda^{+}, 2, \sqsubseteq^{*}, 1,\{S\}, 2\right)$ for every stationary $S \subseteq \lambda^{+}$.

(6) Assuming $\lambda$ is singular, $\square\left(\lambda^{+}\right)+\mathrm{GCH}$ entails $\mathrm{P}^{\bullet}\left(\lambda^{+}, 2, \sqsubseteq, \lambda^{+},\left\{\lambda^{+}\right\}, 2\right)$.

(7) Assuming $\lambda \geq \aleph_{1}, \square\left(\lambda^{+}\right)+\mathrm{GCH}$ entails $\mathrm{P}^{\bullet}\left(\lambda^{+}, 2, \sqsubseteq, 1,\left\{E_{\chi}^{\lambda^{+}}\right\}, 2\right)$ for every $\chi \in \operatorname{Reg}(\lambda)$.

(8) Assuming $\lambda \geq \aleph_{1}$ and $1 \leq \mu<\operatorname{cf}(\lambda), \square\left(\lambda^{+}, \mu\right)+\mathrm{GCH}$ entails $\mathrm{P}^{\bullet}\left(\lambda^{+}, \mu^{+}, \sqsubseteq, 1\right.$, $\left.\left\{E_{\geq \chi}^{\lambda^{+}}\right\}, \mu^{+}\right)$for every $\chi \in \operatorname{Reg}(\operatorname{cf}(\lambda))\left[24\right.$ as well as $\mathrm{P}^{\bullet}\left(\lambda^{+}, \mu^{+}, \sqsubseteq^{*}, 1,\{S\}, \mu^{+}\right)$ for every stationary $S \subseteq \lambda^{+}$.

(9) Assuming $\aleph_{0} \leq \mu<\operatorname{cf}(\lambda)$, $\square^{\text {ind }}\left(\lambda^{+}, \mu\right)+\mathrm{GCH}$ entails $\mathrm{P}^{\bullet}\left(\lambda^{+}, \mu^{\text {ind }}, \sqsubseteq, 1\right.$, $\left.\left\{E_{\geq \chi}^{\lambda^{+}}\right\}, \mu^{+}\right)$for every $\chi \in \operatorname{Reg}(\operatorname{cf}(\lambda))$.

(10) For $\kappa \geq \aleph_{2}$ and a stationary $E \subset \kappa, \square(E)+\diamond(E)$ entails $\mathrm{P}^{\bullet}\left(\kappa, 2, \sqsubseteq^{*}, 1,\{S\}, 2\right)$ for every stationary $S \subseteq \kappa$.

(11) Assuming $\lambda^{<\lambda}=\lambda \geq \aleph_{0}$, after forcing to add a single Cohen subset of $\lambda, \mathrm{P}_{\lambda}^{\bullet}\left(\lambda^{+}, 2, \mathcal{R}, \lambda^{+}, \mathrm{NS}_{\lambda^{+}}^{+}\left\lceil E_{\lambda}^{\lambda^{+}}, 2\right)\right.$ holds with $\mathcal{R}={ }_{\lambda} \sqsubseteq$. If $\square_{\lambda}$ holds in the ground model, then the conclusion holds with $\mathcal{R}=\sqsubseteq$.

(12) Assuming $\lambda^{<\lambda}=\lambda \geq \aleph_{0}$ and $\kappa>\lambda$ strongly inaccessible, after forcing by a $(<\lambda)$-distributive $\kappa$-cc notion of forcing collapsing $\kappa$ to $\lambda^{+}, \mathrm{P}_{\lambda}^{\bullet}\left(\lambda^{+}, \infty, \sqsubseteq, \lambda^{+}\right.$, $\left.\mathrm{NS}_{\kappa}^{+} \uparrow E_{\lambda}^{\lambda^{+}}, 2\right)$ holds.

(13) Assuming $\lambda^{<\lambda}=\lambda \geq \aleph_{1}$ and $\mathrm{CH}_{\lambda}$, after forcing with a $\lambda^{+}$-cc notion of forcing of size $\leq \lambda^{+}$that preserves the regularity of $\lambda$ and is not ${ }^{\lambda} \lambda_{-}$ bounding, $\mathrm{P}_{\lambda}^{\bullet}\left(\lambda^{+}, \infty, \sqsubseteq, \lambda^{+}, \mathrm{NS}_{\lambda^{+}}^{+}\left\lceil E_{\lambda^{\lambda^{+}}}, 2\right)\right.$ holds.

(14) Assuming $\lambda^{<\lambda}=\lambda \geq \aleph_{1}$ and $\mathrm{CH}_{\lambda}$, after forcing with a $\lambda^{+}$-cc notion of forcing of size $\leq \lambda^{+}$that forces $\operatorname{cf}(\lambda)<|\lambda|$ (e.g., Prikry, Magidor, and Radin forcing $), \mathrm{P}_{\lambda}^{\bullet}\left(\kappa, \infty, \sqsubseteq, \kappa, \mathrm{NS}_{\kappa}^{+} \uparrow T, 2\right)$ holds, where $\kappa:=\lambda^{+}$and $T:=E_{\lambda}^{\kappa}$ are defined in the ground model.

(15) For infinite regular cardinals $\theta<\lambda$ satisfying $\lambda^{<\theta}=\lambda$ and $\mathrm{CH}_{\lambda}$, after Lévy-collapsing $\lambda$ to $\theta, \mathrm{P}_{\theta}^{\bullet}\left(\kappa, \infty, \sqsubseteq, \kappa, \mathrm{NS}_{\kappa}^{+} \uparrow T, 2\right)$ holds, where $\kappa:=\lambda^{+}$and $T:=E_{\lambda}^{\kappa}$ are defined in the ground model.

\footnotetext{
${ }^{23}$ Keep in mind the monotonicity properties of the proxy principle, as described in Remark 4.11

${ }^{24}$ For a finite cardinal $\mu, \mu^{+}$stands for $\mu+1$.
} 
(16) If $\lambda=\theta^{+}$for a regular cardinal $\theta$, and $\mathrm{NS} \uparrow E_{\theta}^{\lambda}$ is saturated, then $\mathrm{CH}_{\lambda}$ entails $\mathrm{P}^{\bullet}\left(\lambda^{+}, 2, \lambda \sqsubseteq^{*}, \theta,\left\{E_{\lambda}^{\lambda^{+}}\right\}, 2\right)$.

(17) Assuming $\lambda^{<\lambda}=\lambda \geq \aleph_{1}$ and $\mathrm{CH}_{\lambda}$, if there exists a nonreflecting stationary subset of $E_{\neq \lambda^{+}}^{\lambda^{+}}$, then $\mathrm{P}_{\lambda}^{\bullet}\left(\lambda^{+}, \lambda^{+}, \sqsubseteq,<\lambda,\left\{\lambda^{+}\right\}, 2\right)$ holds, and so does $\mathrm{P}^{\bullet}\left(\lambda^{+}, \lambda^{+}, \sqsubseteq^{*}, 1,\left\{E_{\lambda}^{\lambda^{+}}\right\}, 2\right)$.

(18) Assuming $\lambda=2^{<\lambda}$ is singular and $\square_{\lambda}^{*}+\mathrm{CH}_{\lambda}$, if there exists a nonreflecting stationary subset of $E_{\neq \mathrm{cf}(\lambda)}^{\lambda^{+}}$, then $\mathrm{P}_{\lambda^{2}}^{\bullet}\left(\lambda^{+}, \lambda^{+}, \sqsubseteq, \lambda^{+},\left\{\lambda^{+}\right\}, 2\right)$ holds.

(19) For $\kappa$ strongly inaccessible, if there exists a sequence $\left\langle A_{\alpha} \mid \alpha \in E\right\rangle$ satisfying the hypothesis of Theorem $B$, then $\mathrm{P}^{\bullet}\left(\kappa, \kappa,{ }^{E} \sqsubseteq, 1,\{E\}, 2\right)$ holds.

(20) For $\kappa$ strongly inaccessible, if $\diamond(E)$ holds over some nonreflecting stationary $E \subseteq \kappa$, then $\mathrm{P}^{\bullet}\left(\kappa, \kappa,{ }^{E} \sqsubseteq, \kappa,\{E\}, 2\right)$ holds, and so does $\mathrm{P}^{\bullet}\left(\kappa, \kappa, \sqsubseteq^{*}, 1,\{S\}, 2\right)$ for every nonreflecting stationary $S \subseteq \kappa$.

(21) If $V=L$ and $\kappa$ is not weakly compact, then $\mathrm{P}^{\bullet}(\kappa, 2$, $, \kappa, \mathcal{S}, 2)$ holds for $\mathcal{S}:=\left\{E_{\geq \chi}^{\kappa} \mid \chi \in \operatorname{Reg}(\kappa)\right.$ and $\kappa$ is $(<\chi)$-closed $\}$.

Proof. Each statement asserts that an instance of $\mathrm{P}^{\bullet}(\kappa, \ldots)$ follows from hypotheses that include $\kappa^{<\kappa}=\kappa$. Thus, by Theorem 5.13 (or Corollary 5.16), it suffices to prove $\mathrm{P}^{-}(\kappa, \ldots)$ in each case. Furthermore, for statements where the hypotheses include $\diamond(\kappa)$, we may appeal to Theorem 4.16, so that it suffices to prove $\mathrm{P}^{-}(\kappa, \ldots, 1)$.

(1) By [BR17a, Theorem 3.6] and [BR17a, Corollary 1.12]. (2) By Theorem4.35(1) followed by Lemma 4.32. (3) By [BR17a, Theorem 3.6]. (4) By [BR17a, Corollary 3.9] and [Rin17, Corollary 4.13]. (5) By [Rin17, Corollaries 4.7 and 4.13]. (6) By [BR19a, Corollary 4.22] using $\chi:=\aleph_{0}$, since under GCH every singular cardinal is strong limit. (7) By [Rin17, Corollary 4.5]. (8) By the same proof of Theorem 4.44, and then BR19c, Lemma 3.8]. (9) By Theorem 4.44, (10) This is Corollary 4.19 (2). (11) By Lemma 4.32 above and either Theorem 5.7 or Theorem 4.2(2) of [BR17a]. Note that the cited theorems from [BR17a] require $\lambda$ to be uncountable just to be able to verify $\diamond\left(\lambda^{+}\right)$, whereas, here, we settle for $\mathrm{P}^{-}\left(\lambda^{+}, \ldots\right) \wedge \mathrm{CH}_{\lambda}$. See the proof of Rin15, Theorem 2.3] for the exact details. (12) By [BR19b, Proposition 3.10] (which relies on Theorem 4.35(2) above) followed by Lemma 4.32. (13) By [BR19b, Proposition B(1) and Theorem 3.4] followed by Lemma 4.32 above (cf. Remark 4.33). (14) By [BR19b, Proposition B(2) and Theorem 3.4] followed by Lemma 4.32 above (cf. Remark 4.33) . (15) By BR19b, Proposition 3.9] followed by Lemma 4.32 above (cf. Remark 4.33). (16) By BR17a, Theorem 6.4]. (17) By BR19c, Theorem A]. (18) By [BR19c, Theorem B]. (19) This is Theorem 4.28, (20) By Theorem 4.26 and Corollary 4.27, (21) By [BR17a, Corollary 1.10(5)] or BR19a, Corollary 4.14].

6.1. Basic characteristics of trees. Examining our construction of the $\kappa$-Souslin tree in the proof of Proposition 2.18, we notice that all of the hard work took place when constructing the nonzero limit levels of the tree. It was at those levels that we balanced the normality requirement with the need to bound the size of $T_{\alpha}$ and to seal antichains.

In contrast, the only constraint we addressed at successor levels was the requirement that the tree be ever-branching, in consideration of Lemma 2.1, and we did that by assigning two immediate successors to every node from the previous level 25 But here we have the flexibility to impose some additional features, without affecting the most important global properties of the tree.

Definition 6.2. A streamlined $\kappa$-tree $T$ is said to be:

- binary iff $T \subseteq{ }^{<\kappa} 2$;

\footnotetext{
${ }^{25}$ For two nodes $x, y$ in a streamlined tree $T$, we say that $y$ is an immediate successor of $x$ iff $x \subsetneq y$ and $\operatorname{dom}(y)=\operatorname{dom}(x)+1$.
} 
- $\varsigma$-splitting (for an ordinal $\varsigma<\kappa$ ) iff every node in $T$ admits at least $\varsigma$ many immediate successors;

- splitting iff it is 2-splitting;

- prolific iff, for all $\alpha<\kappa$ and $t \in T_{\alpha},\left\{t^{\curvearrowright} i \mid i<\max \{\omega, \alpha\}\right\} \subseteq T_{\alpha+1}$.

While a $\varsigma$-splitting tree, for any value $\varsigma>2$, cannot be binary, we observe the following implications between properties of a streamlined $\kappa$-tree:

prolific $\Longrightarrow \omega$-splitting $\Longrightarrow$ splitting $\Longrightarrow$ ever-branching.

Referring back to our construction in the proof of Proposition 2.18, we note that the tree constructed there was binary. However, we can easily tweak the construction of the successor levels in order to ensure that the resulting tree ends up being (no longer binary, but rather) prolific and/or $\varsigma$-splitting for some value of $\varsigma<\kappa$, without affecting the validity of any other aspects of the proof. Precisely, for an ordinal $\varsigma<\kappa$, to obtain a $\kappa$-Souslin tree that is prolific and $\varsigma$-splitting, we modify the successor level in the proof of Proposition 2.18, setting, for every $\alpha<\kappa$,

$$
T_{\alpha+1}:=\left\{t^{\curvearrowright} i \mid t \in T_{\alpha}, i<\max \{\alpha, \varsigma, \omega\}\right\} .
$$

Of course, it is no longer true that each $T_{\alpha}$ is a subset of $\alpha 2$ of size $\leq \max \left\{\aleph_{0},|\alpha|\right\}$ as claimed in the original proof, but that was simply a matter of preference. The important constraint, to be maintained for all $\alpha<\kappa$ throughout the recursive construction, is that $T_{\alpha}$ is a subset of ${ }^{\alpha} \kappa$ of size $<\kappa$; this follows at successor levels from the fact that $\varsigma<\kappa$, and at limit levels from regularity of $\kappa$ together with the property $(*)_{\alpha}$ of the construction. Furthermore, $\triangleleft$ must be chosen at the outset to be a well-ordering of ${ }^{<\kappa} \kappa$ (or some larger set) instead of ${ }^{<\kappa} 2$.

As we present the more involved Souslin-tree constructions throughout the rest of this paper, the reader should have no trouble adapting them between binary and prolific/ $\varsigma$-splitting, as desired. Note also that an abstract translation between various kinds of trees is offered in the appendix of BR17b.

But even at the limit levels, there is some degree of flexibility, as witnessed by the two alternatives of the following definition.

Definition 6.3. A streamlined tree $T$ is said to be:

- $\operatorname{slim}$ if $\left|T_{\alpha}\right| \leq \max \left\{|\alpha|, \aleph_{0}\right\}$ for every ordinal $\alpha$.

- $\chi$-complete if, for any $\subsetneq$-increasing sequence $\eta$, of length $<\chi$, of elements of $T$, the limit of the sequence, $\bigcup \operatorname{Im}(\eta)$, is also in $T$.

Notice that the $\kappa$-Souslin tree constructed in the proof of Proposition 2.18 is slim. This is a result of adhering to property $(*)_{\alpha}$ in the definition of levels $T_{\alpha}$ for every $\alpha \in \operatorname{acc}(\kappa)$. Recalling the discussion in Subsection 2.4, there needs to be some stationary $\Gamma \subseteq \kappa$ on which, for every $\alpha \in \Gamma$, not every $\alpha$-branch through $T \uparrow \alpha$ will have its limit placed into $T_{\alpha}$. In the proof of Proposition 2.18 we took the simplest approach by setting $\Gamma:=\operatorname{acc}(\kappa) 26$ A much more complicated approach is taken in BR17b, §5]. In the upcoming treatment, we shall focus on a relatively simple form of a set $\Gamma$, namely, $\Gamma:=E_{>\chi}^{\kappa}$ for some $\chi \in \operatorname{Reg}(\kappa)$. Note that this means that the resulting $\kappa$-Souslin tree would be $\chi$-complete. Nevertheless, a minor tweaking would facilitate obtaining slim $\kappa$-Souslin trees; simply set $\chi:=\omega$ and make sure to impose $\omega_{1}$ as the value of the second parameter of the proxy principle 27

\footnotetext{
${ }^{26}$ As a result of taking this simplest approach, the tree also satisfies the property of being club-regressive (in addition to being slim), as explored in BR17a Proposition 2.3].

${ }^{27}$ The notion of slimness is more prevalent in the context of $\kappa$-Kurepa trees as a property that rules out some trivial examples. Our interest in slim $\kappa$-Souslin trees comes from [BR17b], where we constructed $\kappa$-Souslin trees whose reduced powers are $\kappa$-Kurepa.
} 
6.2. The underlying setup. Throughout the rest of this section, we fix a wellordering $\triangleleft_{\kappa}$ of $H_{\kappa}$. All of the constructions in this section will use hypotheses of the form $\mathrm{P}^{\bullet}(\kappa, \ldots)$ that imply $\kappa^{<\kappa}=\kappa$. Thus, we shall moreover assume that $\operatorname{otp}\left(H_{\kappa}, \triangleleft_{\kappa}\right)=\kappa$.

Definition 6.4. For every $T \in H_{\kappa}$, denote $\beta(T):=0$ unless there is $\beta<\kappa$ such that $T \subseteq{ }^{<\beta} H_{\kappa}$ and $T \nsubseteq{ }^{<\beta} H_{\kappa}$, in which case, we let $\beta(T):=\beta$ for this unique $\beta$. We also let $T_{\beta(T)}:=\{x \in T \mid \operatorname{dom}(x)=\beta(T)\} 28$

We collect here a gallery of actions which we will use throughout the constructions of this section. The reader may skip this definition at the moment, and come back to each of its clauses upon its use.

Definition 6.5. (1) The default extension function, extend : $\left(H_{\kappa}\right)^{2} \rightarrow H_{\kappa}$, is defined as follows. Let extend $(x, T):=x$, unless $\bar{Q}:=\left\{z \in T_{\beta(T)} \mid x \subseteq z\right\}$ is nonempty, in which case, we let extend $(x, T):=\min \left(\bar{Q}, \triangleleft_{\kappa}\right)$.

(2) The function for sealing antichains, anti : $\left(H_{\kappa}\right)^{3} \rightarrow H_{\kappa}$, is defined as follows. Let $\operatorname{anti}(x, T, \mho):=\operatorname{extend}(x, T)$, unless

$$
Q:=\left\{z \in T_{\beta(T)} \mid \exists y \in \mho(x \cup y \subseteq z)\right\}
$$

is nonempty, in which case, we let $\operatorname{anti}(x, T, \mho):=\min \left(Q, \triangleleft_{\kappa}\right)$.

(3) The function for sealing ascending paths, sealpath : $\left(H_{\kappa}\right)^{3} \rightarrow H_{\kappa}$, is defined as follows. Given $(x, T, \mho) \in\left(H_{\kappa}\right)^{3}$, if the set

$Q:=\left\{z \in T_{\beta(T)} \mid x \subseteq z \& \forall y \in \mho[\operatorname{dom}(y)=\operatorname{dom}(x)+1 \Longrightarrow y \nsubseteq z]\right\}$

is nonempty, then let $\operatorname{sealpath}(x, T, \mho):=\min \left(Q, \triangleleft_{\kappa}\right)$. Otherwise, let $\operatorname{sealpath}(x, T, \mho):=\operatorname{extend}(x, T)$.

(4) The function for sealing automorphisms, auto : $\left(H_{\kappa}\right)^{4} \rightarrow H_{\kappa}$, is defined as follows. Given $(x, T, b, \mho)$ : If $x \in T \uparrow(\beta(T)), b$ is a partial function from $T \uparrow(\beta(T))$ to $T \uparrow(\beta(T)), \mho$ is an automorphism of $T \uparrow(\beta(T))$, and the set

$$
Q:=\left\{x_{0} \in \operatorname{dom}(b) \mid \mho\left(x_{0}\right) \neq x_{0}\right\}
$$

is nonempty, then let $x_{0}:=\min \left(Q, \triangleleft_{\kappa}\right), \tilde{x}_{0}:=\operatorname{extend}\left(b\left(x_{0}\right), T\right), \tilde{y}_{0}:=$ $\bigcup_{\gamma<\beta(T)} \mho\left(\tilde{x}_{0}\lceil\gamma)\right.$, and auto $(x, T, b, \mho):=\operatorname{extend}\left(x, T \backslash\left\{\tilde{y}_{0}\right\}\right)$. Otherwise, let auto $(x, T, b, \mho):=\operatorname{extend}(x, T)$.

(5) The function for sealing antichains in derived trees, free : $\left(H_{\kappa}\right)^{4} \rightarrow H_{\kappa}$, is defined as follows 29 Given $(x, T, \vec{b}, \mho) \in\left(H_{\kappa}\right)^{4}$ : If $T$ is a streamlined tree, $\vec{b} \in{ }^{<\kappa} T$ and $\vec{b} \neq \emptyset$, then put $z:=\operatorname{anti}(\emptyset, T(\vec{b}), \mho)$, and consider the following options:

- If $z \in \beta(T)\left(\operatorname{dom}(\vec{b}) H_{\kappa}\right)$ and there exists some $\xi<\operatorname{dom}(\vec{b})$ such that $x \cup(z)_{\xi}$ is in $T$, then let free $(x, T, \vec{b}, \mho):=(z)_{\xi}$ for the least such $\xi$.

- Otherwise, let free $(x, T, \vec{b}, \mho):=\operatorname{extend}(x, T)$.

The following is obvious.

Lemma 6.6 (Extension Lemma). Suppose $x \in T \in H_{\kappa}, \mho, b, \vec{b} \in H_{\kappa}$, and $T$ is a normal subtree of $\leq \beta(T) \kappa$. Then extend $(x, T)$, anti $(x, T, \mho)$, sealpath $(x, T, \mho)$, and free $(x, T, \vec{b}, \mho)$ are elements of $T_{\beta(T)}$ extending $x$. If $T \uparrow \beta(T)$ is moreover everbranching, then auto $(x, T, b, \mho)$ is an element of $T_{\beta(T)}$ extending $x$, as well.

\footnotetext{
${ }^{28}$ If $T$ is a streamlined tree, then this notation coheres with the fourth bullet of Lemma 2.4 but, in general, $T_{\beta(T)}$ may well be empty.

${ }^{29}$ The notation $(z)_{\xi}$ and $T(\vec{b})$ will be introduced in Subsection 6.7 below.
} 
6.3. The prototype construction. In this subsection, we present a construction of a $\kappa$-Souslin tree from the weakest useful instance of the proxy principle.

Corollary 6.7. If $\mathrm{P}^{\bullet}\left(\kappa, \kappa, \sqsubseteq^{*}, 1,\{\kappa\}, \kappa\right)$ holds, then there exists a $\kappa$-Souslin tree.

Proof. Appeal to Theorem 6.8 below with $\chi:=\aleph_{0}$.

All of the subsequent constructions in this section, with the exception of the construction of a uniformly homogeneous tree in Subsection 6.8. will be modeled after the following construction.

Theorem 6.8. Suppose that $\kappa$ is $(<\chi)$-closed for a given $\chi \in \operatorname{Reg}(\kappa)$. Let $\varsigma<\kappa$. If $\mathrm{P}^{\bullet}\left(\kappa, \kappa, \chi \coprod^{*}, 1,\left\{E_{\geq \chi}^{\kappa}\right\}, \kappa\right)$ holds, then there exists a normal, prolific, $\varsigma$-splitting, $\chi$-complete $\kappa$-Souslin tree.

Proof. The proof is very similar in spirit to the one from Proposition 2.18, one just needs to be a little bit more careful.

Suppose $\mathrm{P}^{\bullet}\left(\kappa, \kappa,{ }_{\chi} \sqsubseteq^{*}, 1,\left\{E_{\geq \chi}^{\kappa}\right\}, \kappa\right)$ holds, as witnessed by $\overrightarrow{\mathcal{C}}=\left\langle\mathcal{C}_{\alpha} \mid \alpha<\kappa\right\rangle$ and $\vec{B}=\left\langle B_{\iota} \mid \iota<\kappa\right\rangle$. We shall recursively construct a sequence $\left\langle T_{\alpha} \mid \alpha<\kappa\right\rangle$ of levels whose union will ultimately be the desired tree $T$. In this construction, we shall ensure that for each $\alpha<\kappa$, the level $T_{\alpha}$ will be a subset of ${ }^{\alpha} \kappa$ of size $<\kappa$.

Let $T_{0}:=\{\emptyset\}$, and for all $\alpha<\kappa$, let $T_{\alpha+1}:=\left\{t^{\curvearrowright} i \mid t \in T_{\alpha}, i<\max \{\alpha, \varsigma, \omega\}\right\}$.

Next, suppose that $\alpha<\kappa$ is a nonzero limit ordinal, and that $\left\langle T_{\beta} \mid \beta<\alpha\right\rangle$ has already been defined. Constructing the level $T_{\alpha}$ involves deciding which $\alpha$-branches through $T \uparrow \alpha$ will have their limits placed into the tree. Denote $\Gamma:=E_{\geq \chi}^{\kappa}$. The construction splits into two cases, depending on whether $\alpha \in \Gamma$ :

- Suppose $\alpha \notin \Gamma$. Then we let $T_{\alpha}$ consist of the limits of all $\alpha$-branches through $T \uparrow \alpha$. This construction ensures that the tree will be $\chi$-complete, and as any $\alpha$ branch through $T\lceil\alpha$ is determined by a subset of $T\lceil\alpha$ of cardinality $\operatorname{cf}(\alpha)<\chi$, the $(<\chi)$-closedness of $\kappa$ together with $|T| \alpha \mid<\kappa$ ensures that $\left|T_{\alpha}\right|<\kappa$ at these levels. Normality at level $T_{\alpha}$ is verified by induction: Fixing a closed sequence of ordinals of minimal order-type converging to $\alpha$ enables us to find, for any given $x \in T \uparrow \alpha$, an $\alpha$-branch through $T \uparrow \alpha$ containing $x$, and the limit of such an $\alpha$-branch will necessarily be in $T_{\alpha}$.

- Now suppose $\alpha \in \Gamma$. The idea for ensuring normality at level $T_{\alpha}$ is to attach to each $C \in \mathcal{C}_{\alpha}$ and $x \in T \uparrow \stackrel{\circ}{C}$ some node $\mathbf{b}_{x}^{C} \in{ }^{\alpha} \kappa$ above $x$, and then let

$$
T_{\alpha}:=\left\{\mathbf{b}_{x}^{C} \mid C \in \mathcal{C}_{\alpha}, x \in T \uparrow \stackrel{\circ}{C}\right\} .
$$

By the induction hypothesis, $\left|T_{\beta}\right|<\kappa$ for all $\beta<\alpha$, and by the choice of $\overrightarrow{\mathcal{C}}$ we have $\left|\mathcal{C}_{\alpha}\right|<\kappa$, so that by regularity of $\kappa$ we are guaranteed to end up with $\left|T_{\alpha}\right|<\kappa$.

As for every $C \in \mathcal{C}_{\alpha}$ and $x \in T \uparrow \stackrel{\circ}{C}, \mathbf{b}_{x}^{C}$ will be the limit of some $\alpha$-branch through $T \uparrow \alpha$ that contains $x$, we opt to describe $\mathbf{b}_{x}^{C}$ as the $\operatorname{limit} \bigcup \operatorname{Im}\left(b_{x}^{C}\right)$ of a sequence $b_{x}^{C} \in \prod_{\beta \in \stackrel{\circ}{C} \backslash \operatorname{dom}(x)} T_{\beta}$ such that:

- $b_{x}^{C}(\operatorname{dom}(x))=x$;

- $b_{x}^{C}\left(\beta^{\prime}\right) \subsetneq b_{x}^{C}(\beta)$ for any pair $\beta^{\prime}<\beta$ of ordinals from $(\stackrel{\circ}{C} \backslash \operatorname{dom}(x))$;

- $b_{x}^{C}(\beta)=\bigcup \operatorname{Im}\left(b_{x}^{C} \uparrow \beta\right)$ for all $\beta \in \operatorname{acc}(\stackrel{\circ}{C} \backslash \operatorname{dom}(x))$.

Let $C \in \mathcal{C}_{\alpha}$ be arbitrary. By recursion over $\beta \in \stackrel{\circ}{C}$, we shall assign a value $b_{x}^{C}(\beta)$ in $T_{\beta}$ for all $x \in T \uparrow(\stackrel{\circ}{C} \cap(\beta+1))$.

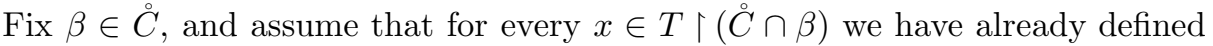

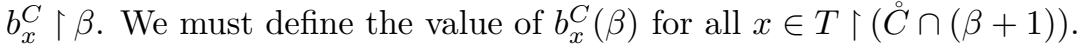

(1) For every $x \in T_{\beta}$, let $b_{x}^{C}(\beta):=x$. We take care of these nodes separately, because for any such node $x$, the sequence $b_{x}^{C}$ is just starting here.

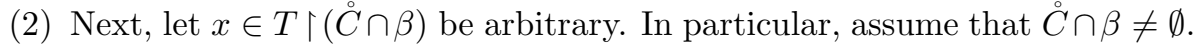




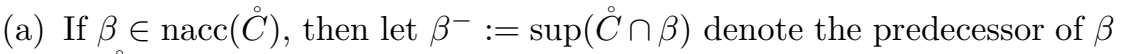
in $\stackrel{\circ}{C}$, and then set

$$
b_{x}^{C}(\beta):= \begin{cases}\operatorname{anti}\left(b_{x}^{C}\left(\beta^{-}\right), T \uparrow(\beta+1), C(\beta)\right), & \text { if } \beta \in B_{0} ; \\ \operatorname{extend}\left(b_{x}^{C}\left(\beta^{-}\right), T \uparrow(\beta+1)\right), & \text { otherwise. }\end{cases}
$$

(b) If $\beta \in \operatorname{acc}(\stackrel{\circ}{C})$, then we let $b_{x}^{C}(\beta):=\bigcup \operatorname{Im}\left(b_{x}^{C} \uparrow \beta\right)$, as promised.

The following is obvious, and is aligned with the microscopic perspective described in requirement (2) of Subsection 2.6.

Dependencies 6.8.1. For any two consecutive points $\beta^{-}<\beta$ of $\operatorname{dom}\left(b_{x}^{C}\right)$, the value of $b_{x}^{C}(\beta)$ is completely determined by $b_{x}^{C}\left(\beta^{-}\right), T \uparrow(\beta+1)$ and $C(\beta)$.

In the case $\beta \in \operatorname{nacc}(\stackrel{\circ}{C})$, since $b_{x}^{C}\left(\beta^{-}\right)$belongs to the normal tree $T \uparrow(\beta+1)$, we infer from the Extension Lemma (Lemma 6.6) that $b_{x}^{C}(\beta)$ is an element of $T_{\beta}$ extending $b_{x}^{C}\left(\beta^{-}\right)$. In the case $\beta \in \operatorname{acc}(\stackrel{\circ}{C})$, the fact that $b_{x}^{C}(\beta)$ belongs to $T_{\beta}$ requires an argument:

Claim 6.8.2. Let $\beta \in \operatorname{acc}(\stackrel{\circ}{C})$. Then $b_{x}^{C}(\beta) \in T_{\beta}$.

Proof. If $\beta \notin \Gamma$, then $T_{\beta}$ was constructed to consist of the limits of all $\beta$-branches through $T \uparrow \beta$, including the limit of the $\beta$-branch determined by $b_{x}^{C} \uparrow \beta$, which is $b_{x}^{C}(\beta)$. Thus, we may assume that $\beta \in \Gamma$.

Since $\beta \in \operatorname{acc}(\stackrel{\circ}{C})$, let us fix $D \in \mathcal{C}_{\beta}$ such that $D \chi \sqsubseteq^{*} C$. As $\Gamma=E_{\geq \chi}^{\kappa}$, in fact,

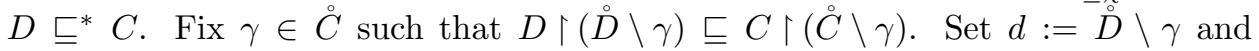
$y:=b_{x}^{C}(\gamma)$. We now prove, by induction on $\delta \in d$, that $b_{x}^{C}(\delta)=b_{y}^{C}(\delta)=b_{y}^{D}(\delta)$.

- Clearly, $b_{x}^{C}(\min (d))=y=b_{y}^{C}(\min (d))=b_{y}^{D}(\min (d))$.

- Suppose $\delta^{-}<\delta$ are successive points of $d$, and $b_{x}^{C}\left(\delta^{-}\right)=b_{y}^{C}\left(\delta^{-}\right)=b_{y}^{D}\left(\delta^{-}\right)$. Then, by Dependencies 6.8.1, also $b_{x}^{C}(\delta)=b_{y}^{C}(\delta)=b_{y}^{D}(\delta)$.

- For $\delta \in \operatorname{acc}(d)$ : If the sequences are identical up to $\delta$, then their limits must be identical.

It follows that $b_{x}^{C}(\beta)=\bigcup_{\delta \in d} b_{x}^{C}(\delta)=\bigcup_{\delta \in d} b_{y}^{D}(\delta)=\mathbf{b}_{y}^{D}$, and by the induction hypothesis $(* *)_{\beta}$, the latter is in $T_{\beta}$. So $b_{x}^{C}(\beta) \in T_{\beta}$, as sought.

This completes the definition of the sequence $b_{x}^{C}$, and thus of its limit $\mathbf{b}_{x}^{C}$, for each $C \in \mathcal{C}_{\alpha}$ and each $x \in T\left\lceil\stackrel{\circ}{C}\right.$. Consequently, the level $T_{\alpha}$ is defined as promised in $(* *)_{\alpha}$.

Claim 6.8.3. Let $\alpha \in \Gamma$ and $t \in T_{\alpha}$. Then there exists $C \in \mathcal{C}_{\alpha}$ such that $\{\delta \in \dot{C} \mid$ $\left.t=\mathbf{b}_{t\lceil\delta}^{C}\right\}$ is a final segment of $\stackrel{\dot{C}}{\text {. }}$.

Proof. By the same analysis from the proof of Claim 6.8.2.

Having constructed all levels of the tree, we then let $T:=\bigcup_{\alpha<\kappa} T_{\alpha}$. It is clear from the construction that $T$ is a normal, prolific, $\varsigma$-splitting, $\chi$-complete streamlined $\kappa$-tree. By Lemma 2.1 to prove that $T$ is $\kappa$-Souslin, it suffices to show that it has no $\kappa$-sized antichains. By Lemma 2.9, it suffices to prove the following.

Claim 6.8.4. Let $A \subseteq T$ be a maximal antichain. Then there exists $\alpha<\kappa$ such that every node of $T_{\alpha}$ extends some element of $A \cap(T\lceil\alpha)$.

Proof. Set $\Omega:=A$ and $p:=\{T, A\}$. By our choice of $\overrightarrow{\mathcal{C}}$ and $\vec{B}$, and recalling Definition [5.9, we now fix $\alpha \in E_{\geq \chi}^{\kappa}$ such that, for all $C \in \mathcal{C}_{\alpha}$,

$$
\sup \left\{\gamma \in \stackrel{\circ}{C} \mid \operatorname{succ}_{1}(\stackrel{\circ}{C} \backslash \gamma) \subseteq B_{0}(\Omega, p, C)\right\}=\alpha .
$$

Let $t \in T_{\alpha}$ be arbitrary. As $\alpha \in E_{\geq \chi}^{\kappa}=\Gamma$, we appeal to Claim 6.8.3, to find

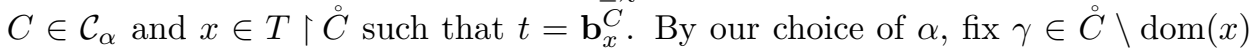


with $\operatorname{succ}_{1}(\stackrel{\circ}{C} \backslash \gamma) \subseteq B_{0}(\Omega, p, C)$. Let $\beta:=\min (\stackrel{\circ}{C} \backslash(\gamma+1))$, so that $\beta \in B_{0}(\Omega, p, C)$. Recalling Definition 5.8 and Proposition $2.12(2)$, we infer that $C(\beta)=A \cap(T\lceil\beta)$ and the latter is a maximal antichain in $T\lceil\beta$.

As $\gamma$ is the predecessor of $\beta$ in $\stackrel{\circ}{C}$, and as $\beta \in B_{0}$, we infer that

$$
b_{x}^{C}(\beta):=\operatorname{anti}\left(b_{x}^{C}(\gamma), T \uparrow(\beta+1), C(\beta)\right) .
$$

Write $\bar{x}:=b_{x}^{C}(\gamma), \bar{T}:=T \uparrow(\beta+1)$ and $\mho:=C(\beta)$. Recalling Definition 6.5(2), we consider the set:

$$
Q:=\left\{z \in \bar{T}_{\beta(\bar{T})} \mid \exists y \in \mho(\bar{x} \cup y \subseteq z)\right\} .
$$

By now, we know that

$$
Q=\left\{z \in T_{\beta} \mid \exists y \in A \cap(T \uparrow \beta)\left(b_{x}^{C}(\gamma) \cup y \subseteq z\right)\right\} .
$$

As $A \cap(T\lceil\beta)$ is a maximal antichain in $T\lceil\beta$, we infer from the normality of $T$ that $Q$ is nonempty, meaning that $b_{x}^{C}(\beta)$ extends some element of $A \cap(T\lceil\beta)$. In particular, $t$ extends some element of $A \cap(T\lceil\alpha)$.

This completes the proof.

6.4. A tree with an ascent path. In BR17b, a gallery of constructions of $\kappa$ Souslin trees with ascent paths was presented. Each of those constructions assumed an instance of the form $\mathrm{P}(\kappa, \ldots, \sigma)$ with $\sigma=\omega$, which, by Proposition 4.31(2), requires the existence of a nonreflecting stationary subset of $E_{\omega}^{\kappa}$. In this section, we present a construction from a weaker instance of the proxy principle which is compatible with reflection, thereby improving an old theorem of Baumgartner (see Dev83]). Note, however, that the objects we obtain here are not as complex and flexible as the ones obtained from the stronger instances in BR17b.

Definition 6.9. Suppose that $T \subseteq{ }^{<\kappa} H_{\kappa}$ is a streamlined $\kappa$-tree. We say that $\vec{f}=\left\langle f_{\alpha} \mid \alpha<\kappa\right\rangle$ is a $\mu$-ascent path through $T$ iff for every pair $\alpha<\beta<\kappa$ :

(1) $f_{\alpha}$ is a function from $\mu$ to $T_{\alpha}$;

(2) $\left\{i<\mu \mid f_{\alpha}(i) \subsetneq f_{\beta}(i)\right\}$ is co-bounded in $\mu$.

Remark 6.10. In the general language of [BR17b, Definition 1.2], the above is called an $\mathcal{F}_{\mu}^{\text {bd }}$-ascent path.

Theorem 6.11. Suppose that $\kappa$ is $(<\chi)$-closed for a given $\chi \in \operatorname{Reg}(\kappa)$, and $\aleph_{0} \leq$ $\mu<\kappa$. Let $\varsigma<\kappa$. If $\mathrm{P}^{\bullet}\left(\kappa, \mu^{\text {ind }}, \sqsubseteq, 1,\left\{E_{>\chi}^{\kappa}\right\}, \kappa\right)$ holds, then there exists a normal, prolific, $\varsigma$-splitting, $\chi$-complete $\kappa$-Souslin tree admitting a $\mu$-ascent path.

Proof. Recalling Definition 5.15] we fix sequences $\overrightarrow{\mathcal{C}}=\left\langle\mathcal{C}_{\alpha} \mid \alpha<\kappa\right\rangle$ and $\vec{B}=\left\langle B_{\iota}\right|$ $\iota<\kappa\rangle$ together witnessing $\mathrm{P}^{\bullet}\left(\kappa, \mu^{+}, \sqsubseteq, 1,\left\{E_{\geq \chi}^{\kappa}\right\}, \kappa\right)$, along with $\langle i(\alpha) \mid \alpha<\kappa\rangle$ satisfying for every $\alpha \in \operatorname{acc}(\kappa)$ :

(1) there exists a canonical enumeration $\left\langle C_{\alpha, i} \mid i(\alpha) \leq i<\mu\right\rangle$ of $\mathcal{C}_{\alpha}$;

(2) for all $i \in[i(\alpha), \mu)$ and $\bar{\alpha} \in \operatorname{acc}\left(\stackrel{C}{\alpha, i}_{\alpha}\right), i \geq i(\bar{\alpha})$ and $C_{\bar{\alpha}, i} \sqsubseteq C_{\alpha, i}$;

(3) $\left\langle\operatorname{acc}\left(\stackrel{i}{C}_{\alpha, i}\right) \mid i(\alpha) \leq i<\mu\right\rangle$ is $\subseteq$-increasing with $\operatorname{acc}(\alpha)=\bigcup_{i \in[i(\alpha), \mu)} \operatorname{acc}\left(\stackrel{\circ}{C}_{\alpha, i}\right)$.

Without loss of generality, we may also assume that:

(4) $0 \in \stackrel{\circ}{C}$ for every $C \in \bigcup_{\alpha \in \operatorname{acc}(\kappa)} \mathcal{C}_{\alpha}$.

Now, the construction of the tree $T$ is identical to that in Theorem 6.8 using $\overrightarrow{\mathcal{C}}$ and $\vec{B}$. We are left with demonstrating that $T$ admits a $\mu$-ascent path.

Referring to the construction, recall that $\Gamma:=E_{\geq \chi}^{\kappa}$ is stationary in $\kappa$, and that for every $\alpha^{\prime} \in \Gamma, C \in \mathcal{C}_{\alpha^{\prime}}$ and $x \in T \uparrow \stackrel{\circ}{C}, \mathbf{b}_{x}^{C}$ is an element of $T_{\alpha^{\prime}}$ extending $x$.

For every $\alpha<\kappa$, let $\alpha^{\prime}:=\min (\Gamma \backslash \alpha)$, and then define $f_{\alpha}: \mu \rightarrow T_{\alpha}$ via

$$
f_{\alpha}(i):=\mathbf{b}_{\emptyset}^{C_{\alpha^{\prime}, \max \left\{i, i\left(\alpha^{\prime}\right)\right\}}} \uparrow \alpha .
$$


Claim 6.11.1. $\left\langle f_{\alpha} \mid \alpha<\kappa\right\rangle$ forms a $\mu$-ascent path through $T$.

Proof. Fix an arbitrary pair $\alpha<\beta$ of ordinals in $\kappa$. Write $\alpha^{\prime}:=\min (\Gamma \backslash \alpha)$ and $\beta^{\prime}:=\min (\Gamma \backslash \beta)$.

- If $\alpha^{\prime}=\beta^{\prime}$, then we trivially get that $f_{\alpha}(i) \subsetneq f_{\beta}(i)$ for all $i<\mu$.

- If $\alpha^{\prime}<\beta^{\prime}$, then $\alpha^{\prime} \in \Gamma \cap \beta^{\prime} \subseteq \operatorname{acc}\left(\beta^{\prime}\right)$. By Clause (3), find a large enough $j<\mu$ such that $\alpha^{\prime} \in \operatorname{acc}\left({\stackrel{\circ}{\beta^{\prime}, i}}\right)$ for all $i \in[j, \mu)$. For any such $i$, we have $C_{\alpha^{\prime}, i} \sqsubseteq C_{\beta^{\prime}, i}$, so that, by Dependencies 6.8.1. $\mathbf{b}_{\emptyset}^{C_{\alpha^{\prime}, i}}=\mathbf{b}_{\emptyset}^{C_{\beta^{\prime}, i}}\left\lceil\alpha^{\prime}\right.$. In particular, $f_{\alpha}(i) \subsetneq f_{\beta}(i)$ on a tail of $i$ 's.

This completes the proof.

Theorem $\mathrm{A}$ is the case $(\lambda, \mu):=\left(\aleph_{1}, \aleph_{0}\right)$ of the following:

Corollary 6.12. Suppose that $\lambda$ is an uncountable cardinal, and $\square\left(\lambda^{+}\right)+\mathrm{CH}_{\lambda}$ holds. For every $\mu \in \operatorname{Reg}(\lambda)$ satisfying $\lambda^{\mu}=\lambda$, there exists a $\lambda^{+}$-Souslin tree admitting a $\mu$-ascent path.

Proof. By Corollary 4.46, Corollary 5.16 and Theorem 6.11, setting $\chi:=\mu$.

Corollary 6.13. In the Harrington-Shelah model [HS85, Theorem A] in which every stationary subset of $E_{\aleph_{0}}^{\aleph_{2}}$ reflects, there also exists an $\aleph_{2}$-Souslin tree with an $\omega$-ascent path.

Proof. The Harrington-Shelah model is a forcing extension of $L$ in which GCH holds, and the first Mahlo cardinal in $L$ becomes $\aleph_{2}$. By standard results in inner model theory, $\square\left(\aleph_{2}\right)$ holds, and hence Corollary 6.12 applies.

6.5. Omitting an ascending path. In [L̈̈c17, Definition 1.3], Lücke considered a weakening of a $\mu$-ascent path which he calls an ascending path of width $\mu$. This is obtained by replacing Clause (2) of Definition 6.9 by:

$\left(2^{\prime}\right)$ there are $i, j<\mu$ such that $f_{\alpha}(i) \subsetneq f_{\beta}(j)$.

Lücke proved (see Lüc17, Theorem 1.9 and subsequent comment]) that, assuming $\lambda^{<\lambda}=\lambda$, for every $\lambda^{+}$-Aronszajn tree $T$, the following are equivalent:

- for every $\Lambda<\lambda$, there is no ascending path of width $\Lambda$ through $T$;

- $T$ is specializable, that is, there exists a notion of forcing $\mathbb{P}$ that does not change the cardinal structure up to and including $\lambda^{+}$, and such that, in $V^{\mathbb{P}}, T$ is the union of $\lambda$ many antichains.

We next show that by decreasing the value of $\nu$ in Theorem 6.8, we can get a $\kappa$-Souslin tree satisfying the additional property of omitting an ascending path.

Theorem 6.14. Suppose that $\kappa$ is $(<\chi)$-closed for a given $\chi \in \operatorname{Reg}(\kappa)$. Let $\varsigma<\kappa$. Given an infinite cardinal $\lambda<\kappa$, put $\mathcal{S}:=\left\{E_{\geq \chi}^{\kappa} \cap E_{>\Lambda}^{\kappa} \mid \Lambda<\lambda\right\}$.

If $\mathrm{P}^{\bullet}\left(\kappa, \kappa, \chi \sqsubseteq^{*}, 1, \mathcal{S}, 2\right)$ holds, then there exists a normal, prolific, $\varsigma$-splitting, $\chi$-complete, $\kappa$-Souslin tree $T$ such that, for all $\Lambda<\lambda$, there is no ascending path of width $\Lambda$ through $T$.

Proof. Suppose $\mathrm{P}^{\bullet}\left(\kappa, \kappa, \chi \sqsubseteq^{*}, 1, \mathcal{S}, 2\right)$ holds, as witnessed by $\overrightarrow{\mathcal{C}}=\left\langle\mathcal{C}_{\alpha} \mid \alpha<\kappa\right\rangle$ and $\vec{B}=\left\langle B_{\iota} \mid \iota<\kappa\right\rangle$. We recursively construct a sequence $\left\langle T_{\alpha} \mid \alpha<\kappa\right\rangle$ of levels whose union will ultimately be the desired tree $T$, as in the proof of Theorem 6.8, using $\Gamma:=E_{\geq \chi}^{\kappa}$. In particular, for every $\alpha \in \Gamma$, we shall have:

$$
T_{\alpha}:=\left\{\mathbf{b}_{x}^{C} \mid C \in \mathcal{C}_{\alpha}, x \in T \uparrow \stackrel{\circ}{C}\right\}
$$


The only difference is in the definition of $b_{x}^{C}$ in the case $\alpha \in \Gamma$, where stage (2)(a) is now done as follows.

$$
b_{x}^{C}(\beta):= \begin{cases}\operatorname{anti}\left(b_{x}^{C}\left(\beta^{-}\right), T \uparrow(\beta+1), C(\beta)\right), & \text { if } \beta \in B_{0} ; \\ \operatorname{sealpath}\left(b_{x}^{C}\left(\beta^{-}\right), T \uparrow(\beta+1), C(\beta)\right), & \text { if } \beta \in B_{1} ; \\ \operatorname{extend}\left(b_{x}^{C}\left(\beta^{-}\right), T \uparrow(\beta+1)\right), & \text { otherwise. }\end{cases}
$$

In all cases, since $b_{x}^{C}\left(\beta^{-}\right)$belongs to the normal tree $T \uparrow(\beta+1)$, we infer from the Extension Lemma (Lemma 6.6) that $b_{x}^{C}(\beta)$ is an element of $T_{\beta}$ extending $b_{x}^{C}\left(\beta^{-}\right)$. Now, it is clear that Dependencies 6.8.1 and Claims 6.8.2 6.8.3 and 6.8.4 are all valid, so that $T:=\bigcup_{\alpha<\kappa} T_{\alpha}$ is a normal, prolific, $\varsigma$-splitting, $\chi$-complete streamlined $\kappa$-Souslin tree. Thus, we are left with verifying the following.

Claim 6.14.1. For every $\Lambda<\lambda, T$ admits no ascending path of width $\Lambda$.

Proof. Fix a nonzero $\Lambda<\lambda$, and suppose toward a contradiction that $\vec{f}=\left\langle f_{\alpha}\right.$ : $\Lambda \rightarrow T_{\alpha}|\alpha<\kappa\rangle$ is an ascending path of width $\Lambda$ through $T$. Let $p:=\vec{f}$ and $\Omega:=\left\{f_{\alpha}(\xi) \mid \xi<\Lambda, \alpha<\kappa\right\}$, so that $p \in H_{\kappa^{+}}$and $\Omega \subseteq H_{\kappa}$. By our choice of $\overrightarrow{\mathcal{C}}$ and $\vec{B}$, and recalling Definition [5.9. we now fix $\alpha \in E_{\geq \chi}^{\kappa} \cap E_{>\Lambda}^{\kappa}$ above $\lambda$ such that $\mathcal{C}_{\alpha}$ is a singleton, say, $\mathcal{C}_{\alpha}=\left\{C_{\alpha}\right\}$, and

$$
\sup \left\{\gamma \in \stackrel{\circ}{C}_{\alpha} \mid \operatorname{succ}_{1}\left(\stackrel{\circ}{C}_{\alpha} \backslash \gamma\right) \subseteq B_{1}\left(\Omega, p, C_{\alpha}\right)\right\}=\alpha .
$$

For every $\xi<\Lambda$, since $f_{\alpha}(\xi) \in T_{\alpha}$ and $\alpha \in \Gamma$, by the construction of the level $T_{\alpha}$, we can fix $x_{\xi} \in T \uparrow \stackrel{\circ}{\alpha}_{\alpha}$ such that $f_{\alpha}(\xi)=\mathbf{b}_{x_{\xi}}^{C_{\alpha}}$. Now let

$$
\delta:=\max \left\{\Lambda^{+}, \sup \left\{\operatorname{dom}\left(x_{\xi}\right) \mid \xi<\Lambda\right\}\right\} .
$$

Since $\operatorname{dom}\left(x_{\xi}\right)<\alpha$ for every $\xi<\Lambda$, and $\Lambda^{+} \leq \lambda<\alpha$, it follows from $\operatorname{cf}(\alpha)>\Lambda$ that $\delta<\alpha$. Thus, we may find a large enough $\beta \in \operatorname{nacc}\left(\stackrel{\circ}{C}_{\alpha}\right) \cap B_{1}\left(\Omega, p, C_{\alpha}\right)$ such that $\beta^{-}:=\sup \left(\dot{C}_{\alpha} \cap \beta\right)$ is greater than $\delta$. Denote $\mho:=C_{\alpha}(\beta)$. It follows that, for all $\xi<\Lambda$ :

- $\beta^{-} \in \dot{C}_{\alpha}$

- $x_{\xi} \in T \uparrow\left(\stackrel{\circ}{C}_{\alpha} \cap \beta^{-}\right)$;

- $f_{\alpha}(\xi)=\mathbf{b}_{x_{\xi}}^{C_{\alpha}}$;

- $C_{\alpha}(\beta)=\mho$.

Since $\beta^{-}+1 \leq \beta<\alpha$ and we have assumed that $\vec{f}$ is an ascending path of width $\Lambda$ through $T$, we can fix $\bar{\xi}<\Lambda$ such that, for some $\xi<\Lambda, f_{\beta^{-+1}}(\xi) \subsetneq f_{\alpha}(\bar{\xi})$. As $\operatorname{dom}\left(x_{\bar{\xi}}\right) \in \stackrel{\circ}{C}_{\alpha} \cap \beta$, we obtain $b_{x_{\bar{\xi}}}^{C_{\alpha}}(\beta) \subsetneq \mathbf{b}_{x_{\bar{\xi}}}^{C_{\alpha}}=f_{\alpha}(\bar{\xi})$. Let us examine how $b_{x_{\bar{\xi}}}^{C_{\alpha}}(\beta)$ was chosen.

As $\beta \in \operatorname{nacc}\left(\stackrel{\circ}{C}_{\alpha}\right) \cap B_{1}$ and $\beta^{-}=\sup \left(\stackrel{\circ}{C}_{\alpha} \cap \beta\right)$, we have

$$
b_{x_{\bar{\xi}}}^{C_{\alpha}}(\beta)=\operatorname{sealpath}\left(b_{x_{\bar{\xi}}}^{C_{\alpha}}\left(\beta^{-}\right), T \uparrow(\beta+1), C_{\alpha}(\beta)\right) .
$$

Returning to Definition 6.5(3) , we consider the following set:

$$
Q:=\left\{z \in T_{\beta} \mid b_{x_{\bar{\xi}}}^{C_{\alpha}}\left(\beta^{-}\right) \subseteq z \& \forall y \in \mho\left[\operatorname{dom}(y)=\beta^{-}+1 \Longrightarrow y \nsubseteq z\right]\right\} .
$$

Recalling Definition 5.8 , let us fix an elementary submodel $\mathcal{M} \prec H_{\kappa^{+}}$satisfying:

$$
p \in \mathcal{M}, \quad \mathcal{M} \cap \kappa=\beta, \text { and } \mathcal{M} \cap \Omega=\mho .
$$

Clearly, $\beta$ is a limit ordinal, and $\mho=\left\{f_{\gamma}(\xi) \mid \xi<\Lambda, \gamma<\beta\right\}$. In particular, $\left\{y \in \mho \mid \operatorname{dom}(y)=\beta^{-}+1\right\}=\left\{f_{\beta^{-+1}}(\xi) \mid \xi<\Lambda\right\}$. Since $T$ is a prolific tree and $\beta^{-}>\delta \geq \Lambda^{+}$, the node $b_{x_{\bar{\xi}}}^{C_{\alpha}}\left(\beta^{-}\right)$must have at least $\Lambda^{+}$many immediate successors in $T_{\beta^{-}+1}$. Thus we can fix $w \in T_{\beta^{-}+1}$ extending $b_{x_{\bar{\xi}}}^{C_{\alpha}}\left(\beta^{-}\right)$that is distinct from $f_{\beta^{-}+1}(\xi)$ for every $\xi<\Lambda$. By normality, we can extend $w$ to some element $z \in T_{\beta}$. It follows that $Q$ is nonempty, so that $b_{x_{\bar{\xi}}}^{C_{\alpha}}(\beta)$ was chosen from $Q$, and 
in particular is incomparable with $f_{\beta^{-+1}}(\xi)$ for every $\xi<\Lambda$. It follows that $f_{\alpha}(\bar{\xi})$ is incomparable with $f_{\beta^{-}+1}(\xi)$ for every $\xi<\Lambda$, contradicting our choice of $\bar{\xi}$ and completing the proof.

This completes the proof.

6.6. Rigid. Compared to the previous subsection, here we strengthen $\mathcal{R}$ from $\chi \sqsubseteq^{*}$ to $\chi \sqsubseteq$, and gain rigidity as a result.

Definition 6.15 (cf. [DJ74, $\S \mathrm{V} .1]$ ). An automorphism of a streamlined tree $T$ is a bijection $\Omega: T \leftrightarrow T$ satisfying $(x \subsetneq y \Longleftrightarrow \Omega(x) \subsetneq \Omega(y)$ ). The identity map is known as the trivial automorphism. A streamlined tree is said to be rigid iff its only automorphism is the trivial one.

Note that any automorphism $\Omega$ of a streamlined tree $T$ is level-preserving, that is, $\Omega\left[T_{\beta}\right]=T_{\beta}$ for every ordinal $\beta$.

Corollary 6.16. If $\mathrm{P}^{\bullet}\left(\kappa, \kappa, \sqsubseteq, 1,\left\{E_{>\lambda}^{\kappa}\right\}, 2\right)$ holds, then there exists a rigid $\kappa$-Souslin tree admitting no ascending path of width $\Lambda$ for every $\Lambda<\lambda$.

Proof. Appeal to the next theorem with $\chi:=\aleph_{0}$.

Theorem 6.17. Suppose that $\kappa$ is $(<\chi)$-closed for a given $\chi \in \operatorname{Reg}(\kappa)$. Let $\varsigma<\kappa$. Given an infinite cardinal $\lambda<\kappa$, put $\mathcal{S}:=\left\{E_{\geq \chi}^{\kappa} \cap E_{>\Lambda}^{\kappa} \mid \Lambda<\lambda\right\}$.

If $\mathrm{P}^{\bullet}(\kappa, \kappa, \chi \sqsubseteq, 1, \mathcal{S}, 2)$ holds, then there exists a normal, prolific, $\varsigma$-splitting, $\chi$ complete, rigid $\kappa$-Souslin tree $T$ such that, for all $\Lambda<\lambda$, there is no ascending path of width $\Lambda$ through $T$.

Proof. Suppose $\mathrm{P}^{\bullet}(\kappa, \kappa, \chi \sqsubseteq, 1, \mathcal{S}, 2)$ holds. Fix a partition of $\kappa$ into stationary sets, $\vec{B}=\left\langle B_{\iota} \mid \iota<\kappa\right\rangle$, such that $B_{2} \cap \operatorname{nacc}(\kappa)=\emptyset$. By Corollary 5.14, then, fix $\overrightarrow{\mathcal{C}}=$ $\left\langle\mathcal{C}_{\alpha} \mid \alpha<\kappa\right\rangle$ that, together with $\vec{B}$, witnesses $\mathrm{P}^{\bullet}\left(\kappa, \kappa,{ }_{\chi} \sqsubseteq, 1, \mathcal{S}, 2\right)$. We recursively construct a sequence $\left\langle T_{\alpha} \mid \alpha<\kappa\right\rangle$ of levels whose union will ultimately be the desired tree $T$, as in the proof of Theorem 6.8, using $\Gamma:=E_{\geq \chi}^{\kappa}$. In particular, for every $\alpha \in \Gamma$, we shall have:

$$
T_{\alpha}:=\left\{\mathbf{b}_{x}^{C} \mid C \in \mathcal{C}_{\alpha}, x \in T\lceil\stackrel{\circ}{C}\} .\right.
$$

The only difference is in the definition of $b_{x}^{C}$ in the case $\alpha \in \Gamma$, where stage (2)(a) is now done as follows.

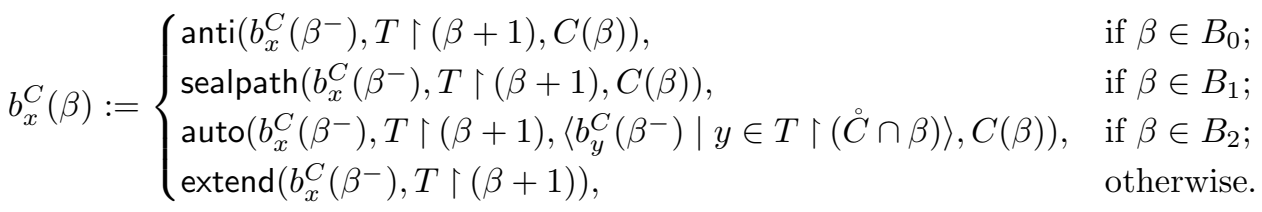

In all cases, since $b_{x}^{C}\left(\beta^{-}\right)$belongs to the normal tree $T \uparrow(\beta+1)$ and $T \uparrow \beta$ is ever-branching, we infer from the Extension Lemma (Lemma [6.6) that $b_{x}^{C}(\beta)$ is an element of $T_{\beta}$ extending $b_{x}^{C}\left(\beta^{-}\right)$.

The following is obvious.

Dependencies 6.17.1. For any two consecutive points $\beta^{-}<\beta$ of $\operatorname{dom}\left(b_{x}^{C}\right)$, the value of $b_{x}^{C}(\beta)$ is completely determined by $b_{x}^{C}\left(\beta^{-}\right), T \uparrow(\beta+1), C(\beta)$, and the map $y \mapsto b_{y}^{C}\left(\beta^{-}\right)$over $T \uparrow(\stackrel{\circ}{C} \cap \beta)$.

Note that, unlike Dependencies 6.8.1, which only involves the value of $C$ at $\beta$, here we have a dependency on $C \uparrow \beta$ as well. The price we pay for this added dependency is that, in order to carry out the proof of Claim 6.8.2 in this context, we require $\overrightarrow{\mathcal{C}}$ to satisfy the stronger $\chi \sqsubseteq$-coherence, rather than merely $\chi \sqsubseteq^{*}$-coherence. 
Claims 6.8.2, 6.8.3 and 6.8.4 remain valid, so that $T:=\bigcup_{\alpha<\kappa} T_{\alpha}$ is a normal, prolific, $\varsigma$-splitting, $\chi$-complete streamlined $\kappa$-Souslin tree. Also, Claim 6.14.1 remains valid, so that for all $\Lambda<\lambda$, there is no ascending path of width $\Lambda$ through $T$. Thus, we are left with proving the following.

Claim 6.17.2. $T$ is rigid.

Proof. Suppose $\Omega: T \rightarrow T$ is a nontrivial automorphism, and we will derive a contradiction. Let $p:=\{\Omega, T\}$, so that $p \in H_{\kappa^{+}}$and $\Omega \subseteq H_{\kappa}$. By our choice of $\vec{B}$ and $\overrightarrow{\mathcal{C}}$, and recalling Definition 5.9, we now fix $\alpha \in E_{\geq \chi}^{\kappa}$ such that $\mathcal{C}_{\alpha}$ is a singleton, say, $\mathcal{C}_{\alpha}=\left\{C_{\alpha}\right\}$, and

$$
\sup \left\{\gamma \in \stackrel{\circ}{C}_{\alpha} \mid \operatorname{succ}_{1}\left(\stackrel{\circ}{C}_{\alpha} \backslash \gamma\right) \subseteq B_{2}\left(\Omega, p, C_{\alpha}\right)\right\}=\alpha .
$$

By Proposition $2.12(3)$, for any $\beta \in B_{2}\left(\Omega, p, C_{\alpha}\right), C_{\alpha}(\beta)=\Omega \uparrow(T \uparrow \beta)$ and the latter is a nontrivial automorphism of $T \uparrow \beta$. In particular, $\Omega \uparrow(T \uparrow \alpha)$ is a nontrivial automorphism of $T \uparrow \alpha$. Thus, by normality, we can let $x_{0}$ be the $\triangleleft_{\kappa}$-least element of $T \uparrow \stackrel{\circ}{C}_{\alpha}$ such that $\Omega\left(x_{0}\right) \neq x_{0}$.

As $\alpha \in \Gamma$, by the nature of the construction of $T_{\alpha}$, it makes sense to consider the particular node $\mathbf{b}_{x_{0}}^{C_{\alpha}}$. Since $\mathbf{b}_{x_{0}}^{C_{\alpha}} \in T_{\alpha}$ and $\Omega$ is an automorphism of $T$, we have $\Omega\left(\mathbf{b}_{x_{0}}^{C_{\alpha}}\right) \in T_{\alpha}$, so that we may choose some $x \in T \uparrow \stackrel{\circ}{C}_{\alpha}$ such that $\Omega\left(\mathbf{b}_{x_{0}}^{C_{\alpha}}\right)=\mathbf{b}_{x}^{C_{\alpha}} 30$

Fix a large enough $\beta \in \operatorname{nacc}\left(\stackrel{\circ}{C}_{\alpha}\right) \cap B_{2}\left(\Omega, p, C_{\alpha}\right)$ such that $\sup \left(\dot{C}_{\alpha} \cap \beta\right)>$ $\max \left\{\operatorname{dom}\left(x_{0}\right), \operatorname{dom}(x)\right\}$. Then $b_{x_{0}}^{C_{\alpha}}(\beta)=\mathbf{b}_{x_{0}}^{C_{\alpha}} \uparrow \beta$ and $b_{x}^{C_{\alpha}}(\beta)=\mathbf{b}_{x}^{C_{\alpha}} \uparrow \beta$. Since $\Omega$ is an automorphism, it follows that

$$
\Omega\left(b_{x_{0}}^{C_{\alpha}}(\beta)\right)=\Omega\left(\mathbf{b}_{x_{0}}^{C_{\alpha}} \uparrow \beta\right)=\Omega\left(\mathbf{b}_{x_{0}}^{C_{\alpha}}\right) \uparrow \beta=\mathbf{b}_{x}^{C_{\alpha}} \uparrow \beta=b_{x}^{C_{\alpha}}(\beta) .
$$

Write $\beta^{-}:=\sup \left(\stackrel{\circ}{C}_{\alpha} \cap \beta\right), \bar{T}:=T \uparrow(\beta+1), \bar{b}:=\left\langle b_{y}^{C_{\alpha}}\left(\beta^{-}\right) \mid y \in T \uparrow\left(\stackrel{\circ}{\alpha}_{\alpha} \cap \beta\right)\right\rangle$ and $\mho:=C_{\alpha}(\beta)$. As $\beta \in \operatorname{nacc}\left(\stackrel{C}{C}_{\alpha}\right) \cap B_{2}$, we infer:

- $b_{x_{0}}^{C_{\alpha}}(\beta)=\operatorname{auto}\left(b_{x_{0}}^{C_{\alpha}}\left(\beta^{-}\right), \bar{T}, \bar{b}, \mho\right)$;

- $b_{x}^{C_{\alpha}}(\beta)=\operatorname{auto}\left(b_{x}^{C_{\alpha}}\left(\beta^{-}\right), \bar{T}, \bar{b}, \mho\right)$.

Consider an arbitrary $z \in\left\{b_{x_{0}}^{C_{\alpha}}\left(\beta^{-}\right), b_{x}^{C_{\alpha}}\left(\beta^{-}\right)\right\}$. Recall Definition 6.5(4), and let us analyze auto $(z, \bar{T}, \bar{b}, \mho)$. We have $z \in \bar{T}$, and $\operatorname{dom}(z)=\beta^{-}<\beta=\beta(\bar{T})$. Since $\beta \in B_{2}\left(\Omega, p, C_{\alpha}\right), \beta$ is a limit ordinal and $\mho$ is the automorphism $\Omega \uparrow(\bar{T} \uparrow \beta)$ of $\bar{T} \uparrow \beta$. Also, $\bar{b}$ is a partial function from $\bar{T} \uparrow(\beta(\bar{T}))$ to $\bar{T} \uparrow(\beta(\bar{T}))$. Since $x_{0} \in \operatorname{dom}(\bar{b})$ and $\Omega\left(x_{0}\right) \neq x_{0}$, the set $Q:=\left\{x_{0} \in \operatorname{dom}(\bar{b}) \mid \mho\left(x_{0}\right) \neq x_{0}\right\}$ is nonempty. By the very choice of $x_{0}$, moreover, $x_{0}=\min \left(Q, \triangleleft_{\kappa}\right)$. Write $\tilde{x}_{0}:=\operatorname{extend}\left(\bar{b}\left(x_{0}\right), \bar{T}\right)$ and $\tilde{y}_{0}:=\bigcup_{\gamma<\beta(\bar{T})} \mho\left(\tilde{x}_{0}\lceil\gamma)\right.$. Since $\beta(\bar{T})=\beta$ is a limit ordinal and $\mho$ is the automorphism $\Omega \uparrow(T \uparrow \beta)$, we obtain $\tilde{y}_{0}=\Omega\left(\tilde{x}_{0}\right)$. Altogether:

- $b_{x_{0}}^{C_{\alpha}}(\beta)=\operatorname{auto}\left(b_{x_{0}}^{C_{\alpha}}\left(\beta^{-}\right), \bar{T}, \bar{b}, \mho\right)=\operatorname{extend}\left(b_{x_{0}}^{C_{\alpha}}\left(\beta^{-}\right), \bar{T} \backslash\left\{\tilde{y}_{0}\right\}\right)$.

- $b_{x}^{C_{\alpha}}(\beta)=\operatorname{auto}\left(b_{x}^{C_{\alpha}}\left(\beta^{-}\right), \bar{T}, \bar{b}, \mho\right)=\operatorname{extend}\left(b_{x}^{C_{\alpha}}\left(\beta^{-}\right), \bar{T} \backslash\left\{\tilde{y}_{0}\right\}\right)$.

Since $x_{0} \subsetneq b_{x_{0}}^{C_{\alpha}}\left(\beta^{-}\right)=\bar{b}\left(x_{0}\right) \subsetneq \operatorname{extend}\left(\bar{b}\left(x_{0}\right), \bar{T}\right)=\tilde{x}_{0}$, we have $\Omega\left(x_{0}\right) \subsetneq \Omega\left(\tilde{x}_{0}\right)=$ $\tilde{y}_{0}$. Thus, by the fact that $\Omega\left(x_{0}\right)$ and $x_{0}$ are two distinct elements of the same level, we infer that $b_{x_{0}}^{C_{\alpha}}\left(\beta^{-}\right)$is incomparable with $\tilde{y}_{0}$. Consequently,

$$
\begin{aligned}
b_{x_{0}}^{C_{\alpha}}(\beta) & =\operatorname{extend}\left(b_{x_{0}}^{C_{\alpha}}\left(\beta^{-}\right), \bar{T} \backslash\left\{\tilde{y}_{0}\right\}\right) \\
& =\operatorname{extend}\left(b_{x_{0}}^{C_{\alpha}}\left(\beta^{-}\right), \bar{T}\right)=\operatorname{extend}\left(\bar{b}\left(x_{0}\right), \bar{T}\right)=\tilde{x}_{0},
\end{aligned}
$$

so that

$$
\Omega\left(b_{x_{0}}^{C_{\alpha}}(\beta)\right)=\Omega\left(\tilde{x}_{0}\right)=\tilde{y}_{0} .
$$

As $b_{x}^{C_{\alpha}}(\beta)=\operatorname{extend}\left(b_{x}^{C_{\alpha}}\left(\beta^{-}\right), \bar{T} \backslash\left\{\tilde{y}_{0}\right\}\right)$, we obtain $b_{x}^{C_{\alpha}}(\beta) \in \bar{T} \backslash\left\{\tilde{y}_{0}\right\}$. So

$$
\Omega\left(b_{x_{0}}^{C_{\alpha}}(\beta)\right) \neq b_{x}^{C_{\alpha}}(\beta) .
$$

This is a contradiction.

\footnotetext{
${ }^{30}$ Recall that $C_{\alpha}$ is the sole element of $\mathcal{C}_{\alpha}$.
} 
This completes the proof.

Theorem B is the case $\lambda:=\aleph_{1}$ of the following corollary.

Corollary 6.18. Suppose that $\kappa$ is a strongly inaccessible cardinal, $\lambda<\kappa$ is regular and uncountable, and there exists a sequence $\left\langle A_{\alpha} \mid \alpha \in S\right\rangle$ such that:

- $S$ is a nonreflecting stationary subset of $E_{\geq \lambda}^{\kappa}$;

- For every $\alpha \in S, A_{\alpha}$ is a cofinal subset of $\alpha$;

- For every cofinal $B \subseteq \kappa$, there exists $\alpha \in S$ for which

$$
\left\{\delta<\alpha \mid \min \left(A_{\alpha} \backslash(\delta+1)\right) \in B\right\}
$$

is stationary in $\alpha$.

Then there is a rigid $\kappa$-Souslin tree admitting no ascending path of width $<\lambda$.

Proof. By Theorem 4.28 in particular, $\mathrm{P}^{-}\left(\kappa, \kappa, \sqsubseteq, 1,\left\{E_{\geq \lambda}^{\kappa}\right\}, 2\right)$ holds. As $\kappa$ is strongly inaccessible, it follows from Theorem 5.13 that $\mathrm{P}^{\bullet}\left(\kappa, \kappa, \sqsubseteq, 1,\left\{E_{\geq \lambda}^{\kappa}\right\}, 2\right)$ holds, as well. Now appeal to Theorem 6.17 with $\chi:=\aleph_{0}$.

6.7. Free. Recall that the square of a tree $\left(T,<_{T}\right)$ is the poset $\left(\hat{T},<_{\hat{T}}\right)$, where

- $\hat{T}:=\left\{\left(t, t^{\prime}\right) \mid t, t^{\prime} \in T, \operatorname{ht}(t)=\operatorname{ht}\left(t^{\prime}\right)\right\}$,

- $\left(s, s^{\prime}\right)<_{\hat{T}}\left(t, t^{\prime}\right)$ iff $s<_{T} t$ and $s^{\prime}<_{T} t^{\prime}$.

By a theorem of Kurepa (see [JW97, Lemma 14.14, Theorem 14.15]), the square of an ever-branching tree of cardinality $\kappa$ has an antichain of size $\kappa$. In particular:

Fact 6.19 (Kurepa, Kur52). The square of a $\kappa$-Souslin tree is not $\kappa$-Souslin.

In contrast, as we shall soon see, the product of two $\kappa$-Souslin trees may still be Souslin. In fact, this is also true for longer products:

Definition 6.20. For a sequence of trees $\left\langle\mathcal{T}^{i} \mid i<\tau\right\rangle$ with $\mathcal{T}^{i}=\left(T^{i},<_{T^{i}}\right)$ for each $i<\tau$, the product $\bigotimes_{i<\tau} \mathcal{T}^{i}$ is defined to be the tree $\left(\hat{T},<_{\hat{T}}\right)$, where:

- $\hat{T}$ consists of all $\vec{t} \in \prod_{i<\tau} T^{i}$ that are level sequences, that is, $i \mapsto \operatorname{ht}_{\mathcal{T}^{i}}(\vec{t}(i))$ is constant over $\tau$

- $\vec{s}<_{\hat{T}} \vec{t}$ iff $\vec{s}(i)<_{T^{i}} \vec{t}(i)$ for every $i<\tau$.

Now, consider a fixed tree $\mathcal{T}=\left(T,<_{T}\right)$. For a node $s \in T$, let us denote $T(s):=\{x \in T \mid x$ is comparable with $s\}$. That is, $T(s)=s_{\downarrow} \cup\{s\} \cup s^{\uparrow}$. Then, for any level sequence $\vec{s}=\left\langle s_{i} \mid i<\tau\right\rangle$ in $\bigotimes_{i<\tau} \mathcal{T}$, we can consider the derived tree $\bigotimes_{i<\tau}\left(T\left(s_{i}\right),<_{T}\right)$ and ask whether it is $\kappa$-Souslin.

In order to bring the notion of derived trees within our framework of streamlined trees, we work with a slightly different definition, as follows.

Notation 6.21 $\left(i^{\text {th }}\right.$-component). For every function $x: \alpha \rightarrow{ }^{\tau} H_{\kappa}$ and every $i<\tau$, let $(x)_{i}: \alpha \rightarrow H_{\kappa}$ denote the sequence $\langle x(\varepsilon)(i) \mid \varepsilon<\alpha\rangle$.

Definition 6.22 (Derived tree, [BR19c, Definition 4.4]). Suppose that $T \subseteq{ }^{<\kappa} H_{\kappa}$ is a streamlined tree, and $\vec{s}=\left\langle s_{i} \mid i<\tau\right\rangle$ is a nonempty sequence of elements of $T$. We let $T(\vec{s})$ stand for the collection of all $x: \alpha \rightarrow{ }^{\tau} H_{\kappa}$ such that:

- $\alpha$ is an ordinal; and

- for all $i<\tau,(x)_{i} \cup s_{i}$ is in $T$.

Note that for a given level sequence $\vec{s}=\left\langle s_{i} \mid i<\tau\right\rangle$ of elements of a streamlined tree $T$, the tree $(T(\vec{s}), \subsetneq)$ is order-isomorphic to $\bigotimes_{i<\tau}\left(T\left(s_{i}\right), \subsetneq\right) 31$

Fact 6.23 (cf. [BR19c, Lemma 4.7]). Suppose that $T \subseteq{ }^{<\kappa} H_{\kappa}$ is a streamlined tree. For every $\chi<\kappa$ and every nonempty sequence $\vec{s} \in<\chi T$ :

\footnotetext{
${ }^{31}$ Recall the last bullet of Lemma 2.4
} 
(1) $T(\vec{s}) \subseteq{ }^{<\kappa} H_{\kappa}$ is a streamlined tree;

(2) If $T$ is normal, then so is $T(\vec{s})$;

(3) If $T$ is a normal $\kappa$-tree and $\kappa$ is $(<\chi)$-closed, then $T(\vec{s})$ is a $\kappa$-tree;

(4) If $T$ is ever-branching, then so is $T(\vec{s})$;

(5) If $\vec{s}=\langle\emptyset\rangle$, then $(T(\vec{s}), \subsetneq)$ is order-isomorphic to $(T, \subsetneq)$ via the isomorphism $x \mapsto(x)_{0}$. Likewise, if $\vec{s}=\langle\emptyset, \emptyset\rangle$, then $(T(\vec{s}), \subsetneq)$ is order-isomorphic to the square of $(T, \subsetneq)$.

By Fact 6.19, if a level-sequence $\vec{s}$ is not injective, then the derived tree $T(\vec{s})$ will not be Souslin. Altogether, this leads us to the following definition.

Definition 6.24 ([BR19c, Definition 4.5]). A streamlined $\kappa$-Souslin tree $T$ is said to be $\chi$-free if for every $\delta<\kappa$ and every nonempty injection $\vec{s} \in{ }^{<\chi} T_{\delta}$, the derived tree $T(\vec{s})$ is again a $\kappa$-Souslin tree.

Remarks 6.25. (1) By [BR17b, Lemma A.7(1)], if there exists a $\chi$-free $\kappa$-Souslin tree, then $\kappa$ is $(<\chi)$-closed.

(2) Instead of $\aleph_{0}$-free, we simply say free.

Corollary 6.26. If $\mathrm{P}^{\bullet}(\kappa, \kappa, \sqsubseteq, \kappa,\{\kappa\}, 2)$ holds, then there is a free $\kappa$-Souslin tree.

Proof. Appeal to the next theorem with $\chi:=\aleph_{0}$.

The main theorem of this subsection reads as follows.

Theorem 6.27. Suppose that $\kappa$ is $(<\chi)$-closed for a given $\chi \in \operatorname{Reg}(\kappa)$. Let $\varsigma<\kappa$. If $\mathrm{P}^{\bullet}\left(\kappa, \kappa, \chi \sqsubseteq, \kappa,\left\{E_{>\chi}^{\kappa}\right\}, 2\right)$ holds, then there exists a normal, prolific, $\varsigma$-splitting, $\chi$-complete, $\chi$-free $\kappa$-Souslin tree.

Proof. Suppose $\mathrm{P}^{\bullet}\left(\kappa, \kappa, \chi \sqsubseteq, \kappa,\left\{E_{>\chi}^{\kappa}\right\}, 2\right)$ holds, as witnessed by $\left\langle\mathcal{C}_{\alpha} \mid \alpha<\kappa\right\rangle$ and $\left\langle B_{\iota} \mid \iota<\kappa\right\rangle$. Let $\pi: \kappa \rightarrow \kappa$ be such that $\beta \in B_{\pi(\beta)}$ for all $\beta<\kappa$. Let $\phi: \kappa \leftrightarrow H_{\kappa}$ witness the isomorphism $(\kappa, \in) \cong\left(H_{\kappa}, \triangleleft_{\kappa}\right)$. Put $\psi:=\phi \circ \pi$.

We recursively construct a sequence $\left\langle T_{\alpha} \mid \alpha<\kappa\right\rangle$ of levels whose union will ultimately be the desired tree $T$, as in the proof of Theorem 6.8 using $\Gamma:=E_{\geq \chi}^{\kappa}$. In particular, for every $\alpha \in \Gamma$, we shall have:

$$
T_{\alpha}:=\left\{\mathbf{b}_{x}^{C} \mid C \in \mathcal{C}_{\alpha}, x \in T \uparrow \stackrel{\circ}{C}\right\} .
$$

The only difference is in the definition of $b_{x}^{C}$ in the case $\alpha \in \Gamma$, where stage (2)(a) is now done as follows.

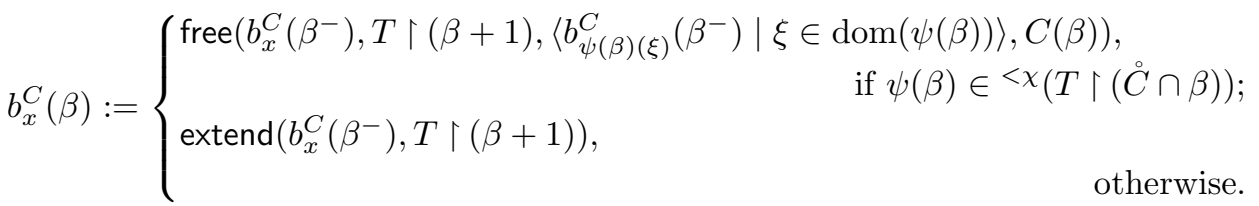

In all cases, since $b_{x}^{C}\left(\beta^{-}\right)$belongs to the normal tree $T \uparrow(\beta+1)$, we infer from the Extension Lemma (Lemma 6.6) that $b_{x}^{C}(\beta)$ is an element of $T_{\beta}$ extending $b_{x}^{C}\left(\beta^{-}\right)$.

The following is obvious.

Dependencies 6.27.1. For any two consecutive points $\beta^{-}<\beta$ of $\operatorname{dom}\left(b_{x}^{C}\right)$, the value of $b_{x}^{C}(\beta)$ is completely determined by $b_{x}^{C}\left(\beta^{-}\right), T \uparrow(\beta+1), C(\beta), \psi(\beta)$, and the map $y \mapsto b_{y}^{C}\left(\beta^{-}\right)$over $T \uparrow(\stackrel{\circ}{C} \cap \beta)$.

It follows that Claims 6.8.2 and 6.8.3 remain valid, where, as in the proof of Theorem 6.17, we appeal to the stronger $\chi \sqsubseteq^{*}$-coherence of $\overrightarrow{\mathcal{C}}$ in order to prove Claim 6.8.2 despite the increased dependency. It follows that $T:=\bigcup_{\alpha<\kappa} T_{\alpha}$ is a normal, prolific, $\varsigma$-splitting, $\chi$-complete streamlined $\kappa$-tree. Thus, we are left with verifying the following. 
Claim 6.27.2. $T$ is a $\chi$-free $\kappa$-Souslin tree.

Proof. We rely throughout on the various clauses of Fact $6.23, T$ is $\kappa$-Souslin iff $T(\langle\emptyset\rangle)$ is $\kappa$-Souslin, and hence it suffices to prove $\chi$-freeness. Fix an arbitrary nonzero ordinal $\tau<\chi$, some $\delta<\kappa$, and an injection $\vec{w}=\left\langle w_{\xi} \mid \xi<\tau\right\rangle \in{ }^{\tau} T_{\delta}$. We need to show that the derived tree $\hat{T}:=T(\vec{w})$ is a $\kappa$-Souslin tree. For this, fix an arbitrary maximal antichain $A$ in $\hat{T}$. Let $\Omega:=A$ and $p:=\{A, T\}$. Fix a transversal $\left\langle C_{\alpha} \mid \alpha<\kappa\right\rangle \in \prod_{\alpha<\kappa} \mathcal{C}_{\alpha}$. Recalling Definition 5.9. we now infer that the following set is stationary:

$$
W:=\left\{\alpha \in E_{\geq \chi}^{\kappa} \mid \mathcal{C}_{\alpha}=\left\{C_{\alpha}\right\} \text { and } \forall i<\alpha\left[\sup \left(\operatorname{nacc}\left(\stackrel{\circ}{C}_{\alpha}\right) \cap B_{i}\left(\Omega, p, C_{\alpha}\right)\right)=\alpha\right]\right\} .
$$

Since $\left\{\alpha \in E_{\geq \chi}^{\kappa} \mid<\chi(T\lceil\alpha) \subseteq \phi[\alpha]\}\right.$ is a club relative to $E_{\geq \chi}^{\kappa}$, let us fix an ordinal $\alpha \in W \backslash(\delta+1)$ such that $<\chi(T\lceil\alpha) \subseteq \phi[\alpha]$.

Let $v \in \hat{T}_{\alpha}$ be arbitrary, and we shall show that it extends some element of $A \cap(\hat{T} \uparrow \alpha)$.

Let $\xi<\tau$ be arbitrary. As $(v)_{\xi} \in T_{\alpha}$ and $\alpha \in W \subseteq \Gamma$, by Claim 6.8.3, there exists some $x_{\xi} \in T \uparrow \dot{C}_{\alpha}$ such that $(v)_{\xi}=\mathbf{b}_{x_{\xi}}^{C_{\alpha}}$. Furthermore, by $\tau<\chi \leq \operatorname{cf}(\alpha)$ and Claim 6.8.3. we may fix a large enough $\gamma \in \stackrel{\circ}{C}_{\alpha} \backslash \delta$ along with $\vec{x}:=\left\langle x_{\xi}\right|$ $\xi<\tau\rangle \in{ }^{\tau} T_{\gamma}$ such that $(v)_{\xi}=\mathbf{b}_{x_{\xi}}^{C_{\alpha}}$ for all $\xi<\tau$. Of course, $\vec{x} \in<\chi(T \uparrow \alpha) \subseteq$ $\phi[\alpha]$. In particular, $i:=\phi^{-1}(\vec{x})$ is $<\alpha$, so that our choice of $\alpha$ guarantees that $\sup \left(\operatorname{nacc}\left(\stackrel{\circ}{C}_{\alpha}\right) \cap B_{i}\left(\Omega, p, C_{\alpha}\right)\right)=\alpha$.

Fix a large enough $\beta \in \operatorname{nacc}\left(\stackrel{\circ}{C}_{\alpha}\right) \cap B_{i}\left(\Omega, p, C_{\alpha}\right)$ with $\gamma \leq \sup \left(\stackrel{\circ}{C}_{\alpha} \cap \beta\right)<\beta<\alpha$. Write $\bar{T}:=T \uparrow(\beta+1), \beta^{-}:=\sup \left(\dot{C}_{\alpha} \cap \beta\right)$, and $\vec{b}:=\left\langle b_{x_{\xi}}^{C_{\alpha}}\left(\bar{\beta}^{-}\right) \mid \xi<\tau\right\rangle$. As $\beta \in B_{i}$, we infer

$$
\psi(\beta)=\phi(\pi(\beta))=\phi(i)=\vec{x} \in^{\tau}\left(T_{\gamma}\right) \subseteq<\chi\left(T \uparrow\left(\check{C}_{\alpha} \cap \beta\right)\right),
$$

so that, for all $x \in T \uparrow\left(\stackrel{\circ}{C}_{\alpha} \cap \beta\right)$,

$$
b_{x}^{C_{\alpha}}(\beta)=\operatorname{free}\left(b_{x}^{C_{\alpha}}\left(\beta^{-}\right), \bar{T}, \vec{b}, C_{\alpha}(\beta)\right) .
$$

Recalling Definition 6.5(5), we must analyze $z:=\operatorname{anti}\left(\emptyset, \bar{T}(\vec{b}), C_{\alpha}(\beta)\right)$. Clearly, $\beta(\bar{T}(\vec{b}))=\beta(\bar{T})=\beta$ and $\bar{T}(\vec{b})=T(\vec{b}) \uparrow(\beta+1)$. Thus, consider the set

$$
Q:=\left\{z \in T(\vec{b})_{\beta} \mid \exists y \in C_{\alpha}(\beta)(y \subseteq z)\right\} .
$$

Let $a: \beta^{-} \rightarrow{ }^{\tau} H_{\kappa}$ be the unique function satisfying $(a)_{\xi}=\vec{b}(\xi)$ for all $\xi<\tau$. For all $\xi<\tau$, we have $x_{\xi} \subseteq b_{x_{\xi}}^{C_{\alpha}}\left(\beta^{-}\right)=(a)_{\xi} \subsetneq \mathbf{b}_{x_{\xi}}^{C_{\alpha}}=(v)_{\xi}$, so that $a=v \uparrow \beta^{-}$is in $\hat{T} \uparrow \beta$. Since $\beta \in B_{i}\left(A,\{A, T\}, C_{\alpha}\right)$, Proposition 2.12(2) entails that $C_{\alpha}(\beta)=A \cap(\hat{T} \uparrow \beta)$ is a maximal antichain in $\hat{T} \uparrow \beta$, and we may pick some $y \in C_{\alpha}(\beta)$ such that $a \cup y \in \hat{T} \uparrow \beta$. Since $T$ is normal, $\hat{T}$ is normal, and hence there exists some $z^{\prime} \in \hat{T}_{\beta}$ such that $a \cup y \subseteq z^{\prime}$. As $a \subseteq z^{\prime}$, we obtain $z^{\prime} \in T(\vec{b})$. Therefore, $Q$ is nonempty, and $z=\min \left(Q, \triangleleft_{\kappa}\right)$.

Notice that $\beta>\gamma \geq \delta$. Since $\left\{w_{\xi} \mid \xi<\tau\right\}$ is an antichain and $w_{\xi} \subsetneq(z)_{\xi}$ for every $\xi<\tau$, it follows that $\left\{(z)_{\xi} \mid \xi<\tau\right\}$ is also an antichain. Thus, we infer that, for all $\xi<\tau$,

$$
\left\{\xi^{\prime}<\tau \mid b_{x_{\xi}}^{C_{\alpha}}\left(\beta^{-}\right) \cup(z)_{\xi^{\prime}} \in T\right\}=\{\xi\} .
$$

Consequently, for all $\xi<\tau, b_{x_{\xi}}^{C_{\alpha}}(\beta)=\operatorname{free}\left(b_{x_{\xi}}^{C_{\alpha}}\left(\beta^{-}\right), \bar{T}, \vec{b}, C_{\alpha}(\beta)\right)=(z)_{\xi}$.

Let $y \in A \cap(\hat{T} \uparrow \beta)$ be a witness to the choice of $z$. Then for all $\xi<\tau$ we infer

$$
(y)_{\xi} \subsetneq(z)_{\xi}=b_{x_{\xi}}^{C_{\alpha}}(\beta) \subsetneq \mathbf{b}_{x_{\xi}}^{C_{\alpha}}=(v)_{\xi} .
$$

This completes the proof. 
Of course, there are cases in which one wants to construct free trees that are slim (or, moreover, regressive) rather than complete. For this, note that the above construction would be equally successful had we set $\Gamma:=\operatorname{acc}(\kappa)$, abandoning the goal of completeness, provided that we assume the stronger coherence relation $\sqsubseteq$ instead of $\chi \sqsubseteq$. In addition, if we require $\left|\mathcal{C}_{\alpha}\right|<\aleph_{1}$ for every $\alpha<\kappa$, then the tree will satisfy $\left|T_{\alpha}\right| \leq|\alpha|$ for all infinite $\alpha<\kappa$. Altogether, we have the following 32

Theorem 6.28. Suppose that $\kappa$ is $(<\chi)$-closed for a given $\chi \in \operatorname{Reg}(\kappa)$.

If $\mathrm{P}^{\bullet}\left(\kappa, \aleph_{1}, \sqsubseteq, \kappa,\left\{E_{\geq \chi}^{\kappa}\right\}, 2\right)$ holds, then there exists a normal, prolific, slim, $\chi$-free $\kappa$-Souslin tree.

\subsection{Uniformly homogeneous and uniformly coherent.}

Definition 6.29. For two elements $x, y$ of $H_{\kappa}$, we define $x * y$ to be the empty set, unless $x, y \in{ }^{<\kappa} H_{\kappa}$ with $\operatorname{dom}(x)<\operatorname{dom}(y)$, in which case $x * y: \operatorname{dom}(y) \rightarrow H_{\kappa}$ is defined by stipulating:

$$
(x * y)(\varepsilon):= \begin{cases}x(\varepsilon), & \text { if } \varepsilon \in \operatorname{dom}(x) \\ y(\varepsilon), & \text { otherwise. }\end{cases}
$$

Definition 6.30. A streamlined $\kappa$-tree $T$ is said to be:

- coherent iff $\{\varepsilon \in \operatorname{dom}(x) \cap \operatorname{dom}(y) \mid x(\varepsilon) \neq y(\varepsilon)\}$ is finite for all $x, y \in T$;

- uniformly homogeneous iff for all $y \in T$ and $x \in T \uparrow \operatorname{dom}(y), x * y$ is in $T$;

- uniformly coherent iff it is coherent and uniformly homogeneous.

Corollary 6.31. If $\mathrm{P}^{\bullet}(\kappa, \kappa, \sqsubseteq, \kappa,\{\kappa\}, \kappa)$ holds, then there exists a uniformly homogeneous $\kappa$-Souslin tree.

Proof. Appeal to the next theorem with $\chi:=\aleph_{0}$.

Theorem 6.32. Suppose that $\kappa$ is $(<\chi)$-closed for a given $\chi \in \operatorname{Reg}(\kappa)$. Let $\varsigma<\kappa$. If $\mathrm{P}^{\bullet}\left(\kappa, \kappa, \chi \sqsubseteq, \kappa,\left\{E_{\geq \chi}^{\kappa}\right\}, \kappa\right)$ holds, then there exists a normal, prolific, $\varsigma$-splitting, $\chi$-complete, uniformly homogeneous $\kappa$-Souslin tree.

Proof. Suppose $\mathrm{P}^{\bullet}\left(\kappa, \kappa, \chi \sqsubseteq, \kappa,\left\{E_{\geq \chi}^{\kappa}\right\}, \kappa\right)$ holds, as witnessed by $\left\langle\mathcal{C}_{\alpha} \mid \alpha<\kappa\right\rangle$ and $\left\langle B_{\iota} \mid \iota<\kappa\right\rangle$. We may assume that $0 \in \stackrel{\circ}{C}$ for every $C \in \bigcup_{\alpha \in \operatorname{acc}(\kappa)} \mathcal{C}_{\alpha}$. Let $\pi: \kappa \rightarrow \kappa$ be such that $\beta \in B_{\pi(\beta)}$ for all $\beta<\kappa$. Let $\phi: \kappa \leftrightarrow H_{\kappa}$ witness the isomorphism $(\kappa, \in) \cong\left(H_{\kappa}, \triangleleft_{\kappa}\right)$. Put $\psi:=\phi \circ \pi$.

We recursively construct a sequence $\left\langle T_{\alpha} \mid \alpha<\kappa\right\rangle$ of levels whose union will ultimately be the desired tree $T$. Denote $\Gamma:=E_{\geq \chi}^{\kappa}$. For every $\alpha \in \Gamma$ and $C \in \mathcal{C}_{\alpha}$, we shall define an $\alpha$-branch $\mathbf{b}^{C}$ through $T \uparrow \alpha$; then, we will ensure that, for every $\alpha \in \operatorname{acc}(\kappa)$ :

$$
T_{\alpha}= \begin{cases}\left\{x * \mathbf{b}^{C} \mid C \in \mathcal{C}_{\alpha}, x \in T \uparrow \alpha\right\}, & \text { if } \alpha \in \Gamma ; \\ \left\{t \in{ }^{\alpha} H_{\kappa} \mid \forall \bar{\alpha}<\alpha\left(t \uparrow \bar{\alpha} \in T_{\bar{\alpha}}\right)\right\}, & \text { otherwise. }\end{cases}
$$

Here we go. Let $T_{0}:=\{\emptyset\}$, and for all $\alpha<\kappa$, let $T_{\alpha+1}:=\left\{t^{\curvearrowright} i \mid t \in T_{\alpha}\right.$, $i<\max \{\alpha, \varsigma, \omega\}\}$. Next, suppose that $\alpha \in \operatorname{acc}(\kappa)$ and that $\left\langle T_{\beta} \mid \beta<\alpha\right\rangle$ has already been defined. The construction splits into two cases:

- If $\alpha \in \operatorname{acc}(\kappa) \backslash \Gamma$, then $\operatorname{cf}(\alpha)<\chi$, and we let $T_{\alpha}$ consist of the limits of all $\alpha$-branches through $T \uparrow \alpha$. This coheres with $(\otimes)_{\alpha}$ and ensures that the outcome tree will be $\chi$-complete.

- Now suppose $\alpha \in \Gamma$. For every $C \in \mathcal{C}_{\alpha}$, we shall define a node $\mathbf{b}^{C}$, and then we would let:

$$
T_{\alpha}:=\left\{x * \mathbf{b}^{C} \mid C \in \mathcal{C}_{\alpha}, x \in T \uparrow \alpha\right\}
$$

\footnotetext{
${ }^{32}$ For more results in this direction, see [BR17b, $\left.\S 6\right]$.
} 
To obtain $\mathbf{b}^{C}$, we define a sequence $b^{C} \in \prod_{\beta \in C} T_{\beta}$ by recursion. Let $b^{C}(0):=\emptyset$. Next, suppose $\beta^{-}<\beta$ are successive points of $\stackrel{\circ}{C}$, and $b^{C}\left(\beta^{-}\right)$has already been defined. Let

$$
b^{C}(\beta):= \begin{cases}b^{C}\left(\beta^{-}\right) * \operatorname{anti}\left(\psi(\beta) * b^{C}\left(\beta^{-}\right), T \uparrow(\beta+1), C(\beta)\right), & \text { if } \psi(\beta) \in T \uparrow \beta^{-} ; \\ \operatorname{extend}\left(b^{C}\left(\beta^{-}\right), T \uparrow(\beta+1)\right), & \text { otherwise. }\end{cases}
$$

If $\psi(\beta) \in T \uparrow \beta^{-}$, then, by the induction hypothesis $(\otimes)_{\beta}, \psi(\beta) * b^{C}\left(\beta^{-}\right)$belongs to the normal tree $T \uparrow(\beta+1)$, so that the Extension Lemma (Lemma 6.6) together with $(\otimes)_{\beta}$ imply $b^{C}(\beta)$ is an element of $T_{\beta}$ extending $b^{C}\left(\beta^{-}\right)$. If $\psi(\beta) \notin T\left\lceil\beta^{-}\right.$, then again the Extension Lemma implies that $b^{C}(\beta)$ is in $T_{\beta}$ and extends $b^{C}\left(\beta^{-}\right)$.

The following is obvious.

Dependencies 6.32.1. For any two consecutive points $\beta^{-}<\beta$ of $\operatorname{dom}\left(b^{C}\right)$, the value of $b^{C}(\beta)$ is completely determined by $b^{C}\left(\beta^{-}\right), \psi(\beta), T \uparrow(\beta+1)$ and $C(\beta)$.

In the case $\beta \in \operatorname{acc}(\stackrel{\circ}{C})$, we let $b^{C}(\beta):=\bigcup\left(\operatorname{Im}\left(b^{C} \uparrow \beta\right)\right)$. The fact that the latter belongs to $T_{\beta}$ follows from Dependencies $6.32 .1(\otimes)_{\beta}$ and $\chi \sqsubseteq$-coherence.

This completes the definition of the level $T_{\alpha}$.

Having constructed all levels of the tree, we then let $T:=\bigcup_{\alpha<\kappa} T_{\alpha}$. It is clear that $T$ is a normal, prolific, $\varsigma$-splitting, $\chi$-complete and uniformly homogeneous streamlined $\kappa$-tree.

Claim 6.32.2. Let $A \subseteq T$ be a maximal antichain. Then there exists $\alpha<\kappa$ such that every node of $T_{\alpha}$ extends some element of $A \cap(T \uparrow \alpha)$.

Proof. Let $\Omega:=A$ and $p:=\{T, A\}$. Consider the club $D:=\{\alpha \in \operatorname{acc}(\kappa) \mid$ $T\lceil\alpha \subseteq \phi[\alpha]\}$, and then pick $\alpha \in D \cap \Gamma$ such that, for all $C \in \mathcal{C}_{\alpha}$ and $i<\alpha$,

$$
\sup \left(\operatorname{nacc}(\stackrel{\circ}{C}) \cap B_{i}(\Omega, p, C)\right)=\alpha .
$$

Now, let $v \in T_{\alpha}$ be arbitrary, and we shall show that $v$ extends some element of $A \cap(T \uparrow \alpha)$. As $\alpha \in \Gamma$, let us fix $C \in \mathcal{C}_{\alpha}$ and $x \in T \uparrow \alpha$ such that $v=x * \mathbf{b}^{C}$. As $\alpha \in D$ and $x \in T\lceil\alpha$, we may also find $i<\alpha$ such that $\phi(i)=x$.

Fix $\beta \in \operatorname{nacc}(\stackrel{\circ}{C}) \cap B_{i}(\Omega, p, C)$ large enough to ensure that $\beta^{-}>\operatorname{dom}(x)$ for

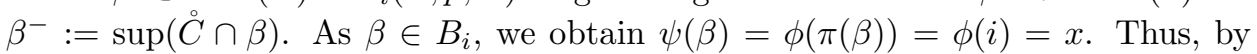
writing $\bar{x}:=x * b^{C}\left(\beta^{-}\right), \bar{T}:=T \uparrow(\beta+1)$ and $\mho:=C(\beta)$, we get that

$$
b^{C}(\beta):=b^{C}\left(\beta^{-}\right) * \operatorname{anti}(\bar{x}, \bar{T}, \mho) .
$$

Recalling Definition 6.5)(2), we consider the set:

$$
Q:=\left\{z \in \bar{T}_{\beta(\bar{T})} \mid \exists y \in \mho(\bar{x} \cup y \subseteq z)\right\} .
$$

By now, we know that

$$
Q=\left\{z \in T_{\beta} \mid \exists y \in A \cap(T \uparrow \beta)\left(\left(x * b^{C}\left(\beta^{-}\right)\right) \cup y \subseteq z\right)\right\} .
$$

Since $\beta \in B_{i}(A,\{T, A\}, C)$, we infer from Proposition $2.12(2)$ that $C(\beta)=A \cap(T \uparrow \beta)$ is a maximal antichain in $T \uparrow \beta$. So $Q$ is nonempty, and $b^{C}(\beta)=b^{C}\left(\beta^{-}\right) * z$, for $z=\min \left(Q, \triangleleft_{\kappa}\right)$. Pick $y \in A \cap(T \uparrow \beta)$ witnessing that $z \in Q$. Then:

$$
v=x * \mathbf{b}^{C} \supsetneq=x * b^{C}(\beta)=x *\left(b^{C}\left(\beta^{-}\right) * z\right)=z \supsetneq y,
$$

as sought.

This completes the proof.

Theorem 6.33. If $\kappa$ is $(<\chi)$-closed, then $\mathrm{P}^{\bullet}\left(\kappa, \kappa, \chi \sqsubseteq^{*}, \kappa,\left\{E_{\geq \chi}^{\kappa}\right\}, \kappa\right)$ implies the existence of a normal, prolific, $\chi$-complete, uniformly homogeneous $\kappa$-Souslin tree.

Proof. By Theorem 6.32, using Theorem 4.39 
Definition 6.34. For a streamlined $\kappa$-tree $T$ and a subset $\Delta \subseteq \kappa$, we say that $T$ is $\Delta$-similar iff, for all $\delta \in \Delta \cap \operatorname{acc}(\kappa)$ and $x, y \in T_{\delta}, \sup \{\varepsilon<\delta \mid x(\varepsilon) \neq y(\varepsilon)\}<\delta$.

It is easy to see that a streamlined $\kappa$-tree is coherent iff it is $\kappa$-similar. It is also easy to see that in the construction of Theorem 6.32, the outcome tree $T$ is $\Delta$-similar for the set $\Delta:=\left\{\delta \in \Gamma|| \mathcal{C}_{\delta} \mid=1\right\}$. Therefore, we get:

Theorem 6.35. Suppose $\mathrm{P}^{\bullet}(\kappa, 2, \sqsubseteq, \kappa,\{\kappa\}, 2)$ holds. Then there exists a normal, slim, prolific, club-regressive, uniformly coherent $\kappa$-Souslin tree.

Remark 6.36. The definition of club-regressive trees may be found in [BR17a, §2]. For further analysis, see BR17a, Proposition 2.5].

\section{SOME OPEN PROBLEMS}

It follows from Theorem 6.1(11) that after forcing to add a single Cohen real over a model of $\mathrm{CH}+\neg \diamond\left(\aleph_{1}\right)$, a very strong instance of $\mathrm{P}^{\bullet}\left(\aleph_{1}, \ldots\right)$ holds, while $\diamond\left(\aleph_{1}\right)$ fails. We conjecture that Radin forcing can be used to answer the following question in the affirmative:

Question 7.1. Is a conjunction of the form $\mathrm{P}^{\bullet}(\kappa, \ldots)+\neg \diamond(\kappa)$ consistent for $\kappa$ inaccessible?

Corollaries 4.19 and 4.36 leave the following question open:

Question 7.2. Assume $\square(\kappa)+\diamond(\kappa)$.

(i) Does $\mathrm{P}^{-}(\kappa, 2, \sqsubseteq, 1,\{\kappa\}, 2)$ hold?

(ii) Does (the weaker instance) $\mathrm{P}^{-}(\kappa, \kappa, \sqsubseteq, 1,\{\kappa\}, \kappa)$ hold?

By Clauses (1) and (5) of Theorem6.1, the answer to (i) is affirmative for $\kappa=\aleph_{1}$ and $\kappa=\lambda^{+}$such that $\lambda \geq \beth_{\omega}$. By [Rin17, Theorem 4.3 and Corollary 2.5], the answer to (i) is also affirmative for $\kappa=\lambda^{+}$such that $\lambda^{\aleph_{0}}=\lambda$. Thus, the remaining cases are $\kappa$ inaccessible or $\aleph_{1}<\kappa<\beth_{\omega}$. For $\kappa$ strongly inaccessible, a sufficient condition for (ii) is given by Theorem 4.28 ,

Our next question has to do with the parameter $\theta$ of the proxy principle.

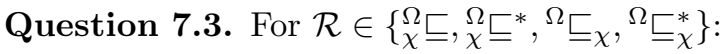

(i) Does $\mathrm{P}^{-}(\kappa, \mu, \mathcal{R}, 1,\{\kappa\}, \nu)$ entail $\mathrm{P}^{-}(\kappa, \mu, \mathcal{R}, \kappa,\{\kappa\}, \nu)$ ?

(ii) Does $\mathrm{P}^{\bullet}(\kappa, \mu, \mathcal{R}, 1,\{\kappa\}, \nu)$ entail $\mathrm{P}^{\bullet}(\kappa, \mu, \mathcal{R}, \kappa,\{\kappa\}, \nu)$ ?

Some partial answers to (ii) include [BR19a, Lemma 3.7], [BR19c, Lemma 3.20] and LR19, Lemmas 3.8 and 3.9], but there are more findings which haven't yet been published. Motivated by [LR19, Lemma 2.13], we hope to prove that, for $\kappa$ inaccessible, $\mathrm{P}^{\bullet}(\kappa, 2, \sqsubseteq, 1,\{\kappa\}, 2)$ entails the existence of graph of size and chromatic number $\kappa$ all of whose smaller subgraphs are countably chromatic.

Question 7.4. Assume GCH. For a regular uncountable cardinal $\lambda$ :

(i) Does $\square_{\lambda}$ entail the existence of a uniformly coherent $\lambda^{+}$-Souslin tree?

(ii) Does the existence of a nonreflecting stationary subset of $E_{<\lambda}^{\lambda^{+}}$entail the existence of a $\lambda$-free $\lambda^{+}$-Souslin tree?

By [BR19a, Theorem C] and [BR19c, Theorem B], the answer is affirmative for the analogous questions concerning $\lambda$ singular. Also, note that an affirmative answer to Question 7.3(ii) would provide an affirmative answer to Question 7.4.

Question 7.5. (i) May $\boldsymbol{\beta}(\kappa)$ be waived from the hypothesis of Theorem 4.16?

(ii) May the hypothesis $\mathrm{P}_{\xi}^{-}(\kappa, \mu, \mathcal{R}, \theta, \mathcal{S}, \nu)$ of Theorem 5.13 be weakened to $\mathrm{P}_{\xi}^{-}(\kappa, \mu, \mathcal{R}, \theta, \mathcal{S}, \nu, 1) ? 33$

\footnotetext{
${ }^{33}$ Recall Convention 4.18
} 


\section{ACKNOWLEDGements}

The first author was supported by the Center for Absorption in Science, Ministry of Aliyah and Integration, State of Israel. The second author was partially supported by the European Research Council (grant agreement ERC-2018-StG 802756) and by the Israel Science Foundation (grant agreement 2066/18).

This paper is submitted to the proceedings of 50 Years of Set Theory in Toronto. The two authors first met at the Toronto Set Theory Seminar, when Brodsky was a Ph.D. student of Stevo Todorcevic and Rinot was a Fields-Ontario postdoctoral fellow of Ilijas Farah, Stevo Todorcevic and Bill Weiss.

We thank James Cummings, Moti Gitik, Yair Hayut, Adi Jarden, Menachem Kojman, Chris Lambie-Hanson, Philipp Lücke, Matti Rubin (of blessed memory), Stevo Todorcevic, and Bill Weiss, with whom we had stimulating discussions on the subject matter of this project throughout the years.

\section{REFERENCES}

[AS93] Uri Abraham and Saharon Shelah. A $\Delta_{2}^{2}$ well-order of the reals and incompactness of $L\left(Q^{M M}\right)$. Ann. Pure Appl. Logic, 59(1):1-32, 1993.

[Alv99] Carlos Alvarez. On the history of Souslin's problem. Arch. Hist. Exact Sci., 54(3):181242, 1999.

[BR17a] Ari Meir Brodsky and Assaf Rinot. A microscopic approach to Souslin-tree constructions. Part I. Ann. Pure Appl. Logic, 168(11):1949-2007, 2017.

[BR17b] Ari Meir Brodsky and Assaf Rinot. Reduced powers of Souslin trees. Forum Math. Sigma, 5(e2):1-82, 2017.

[BR19a] Ari Meir Brodsky and Assaf Rinot. Distributive Aronszajn trees. Fund. Math., 245(3):217-291, 2019.

[BR19b] Ari Meir Brodsky and Assaf Rinot. More notions of forcing add a Souslin tree. Notre Dame J. Form. Log., 60(3):437-455, 2019.

[BR19c] Ari Meir Brodsky and Assaf Rinot. A remark on Schimmerling's question. Order, 36(3):525-561, 2019

[BS86] Shai Ben-David and Saharon Shelah. Souslin trees and successors of singular cardinals. Ann. Pure Appl. Logic, 30(3):207-217, 1986.

[Cum97] James Cummings. Souslin trees which are hard to specialise. Proc. Amer. Math. Soc., 125(8):2435-2441, 1997.

[Dev78] Keith J. Devlin. A note on the combinatorial principles $\diamond(E)$. Proc. Amer. Math. Soc., 72(1):163-165, 1978.

[Dev83] Keith J. Devlin. Reduced powers of $\aleph_{2}$-trees. Fund. Math., 118(2):129-134, 1983.

[Dev84] Keith J. Devlin. Constructibility. Perspectives in Mathematical Logic. Springer-Verlag, Berlin, 1984.

[DJ74] Keith J. Devlin and Hảvard Johnsbràten. The Souslin problem. Lecture Notes in Mathematics, Vol. 405. Springer-Verlag, Berlin, 1974.

[Dra74] Frank R. Drake. Set theory - an introduction to large cardinals, volume 76 of Studies in Logic and the Foundations of Mathematics. North-Holland Publishing Co., Amsterdam, 1974.

[Gre76] John Gregory. Higher Souslin trees and the generalized continuum hypothesis. J. Symbolic Logic, 41(3):663-671, 1976.

[HJ99] Karel Hrbacek and Thomas Jech. Introduction to set theory, volume 220 of Monographs and Textbooks in Pure and Applied Mathematics. Marcel Dekker, Inc., New York, third edition, 1999

[HS85] Leo Harrington and Saharon Shelah. Some exact equiconsistency results in set theory. Notre Dame J. Formal Logic, 26(2):178-188, 1985.

[HSW10] Michael Holz, Karsten Steffens, and Edmund Weitz. Introduction to cardinal arithmetic. Modern Birkhäuser Classics. Birkhäuser Verlag, Basel, 2010. Reprint of the 1999 original.

[Jec67] Tomáš Jech. Non-provability of Souslin's hypothesis. Comment. Math. Univ. Carolinae, 8(2):291-305, 1967.

[Jen68] Ronald B Jensen. Souslin's hypothesis is incompatible with V=L. Notices Amer. Math. Soc., 15(6):935, 1968.

[Jen72] R. Björn Jensen. The fine structure of the constructible hierarchy. Ann. Math. Logic, 4:229-308; erratum, ibid. 4:443, 1972. With a section by Jack Silver. 
[Jus01] Winfried Just. \&-like principles under CH. Fund. Math., 170(3):247-256, 2001.

[JW97] Winfried Just and Martin Weese. Discovering modern set theory. II, volume 18 of Graduate Studies in Mathematics. American Mathematical Society, Providence, RI, 1997. Set-theoretic tools for every mathematician.

[Kan11] Akihiro Kanamori. Historical remarks on Suslin's problem. In Set theory, arithmetic, and foundations of mathematics: theorems, philosophies, volume 36 of Lect. Notes Log., pages 1-12. Assoc. Symbol. Logic, La Jolla, CA, 2011.

[KLY07] Bernhard König, Paul Larson, and Yasuo Yoshinobu. Guessing clubs in the generalized club filter. Fund. Math., 195(2):177-191, 2007.

[KS93] Menachem Kojman and Saharon Shelah. $\mu$-complete Suslin trees on $\mu^{+}$. Archive for Mathematical Logic, 32:195-201, 1993.

[Kun80] Kenneth Kunen. Set theory, volume 102 of Studies in Logic and the Foundations of Mathematics. North-Holland Publishing Co., Amsterdam-New York, 1980. An introduction to independence proofs.

[Kur35] Georges (Đuro) Kurepa. Ensembles ordonnés et ramifiés. Thèses de l'entre-deuxguerres, 175, 1935. http://www.numdam.org/item/THESE_1935__175__1_0/ Publications de l'Institut Mathématique Beograd, 4:1-138, 1935. Included (without the Appendix) in Kur96, pp. 12-114].

[Kur38] Georges Kurepa. Ensembles linéaires et une classe de tableaux ramifiés (tableaux ramifiés de M. Aronszajn). Publ. Math. Univ. Belgrade, 6-7:129-160, 1938. Available (excluding pp. 135-138) at http://elib.mi.sanu.ac.rs/files/journals/publ/6/14.pdf. Included in Kur96, pp. 115-142].

[Kur52] Đuro Kurepa. Sur une propriété caractéristique du continu linéaire et le problème de Suslin. Acad. Serbe Sci. Publ. Inst. Math., 4:97-108, 1952. http://elib.mi.sanu.ac.rs/files/journals/publ/10/10.pdf. Included in Kur96. pp. 329-339].

[Kur96] Đuro Kurepa. Selected papers of Đuro Kurepa. Matematički Institut SANU, Belgrade, 1996. Edited and with commentaries by Aleksandar Ivić, Zlatko Mamuzić, Žarko Mijajlović and Stevo Todorčević.

[Lar99] Paul Larson. An $\mathbf{S}_{\max }$ variation for one Souslin tree. J. Symbolic Logic, 64(1):81-98, 1999.

[Lam17a] Chris Lambie-Hanson. Aronszajn trees, square principles, and stationary reflection. Mathematical Logic Quarterly, 63(3-4):265-281, 2017.

[Lam17b] Chris Lambie-Hanson. Squares and narrow systems. J. Symb. Log., 82(3):834-859, 2017.

[Lev02] Azriel Levy. Basic set theory. Dover Publications, Inc., Mineola, NY, 2002.

[LL18] Chris Lambie-Hanson and Philipp Lücke. Squares, ascent paths, and chain conditions. J. Symb. Log., 83(4):1512-1538, 2018.

[LR19] Chris Lambie-Hanson and Assaf Rinot. Reflection on the coloring and chromatic numbers. Combinatorica, 39(1):165-214, 2019.

[Lüc17] Philipp Lücke. Ascending paths and forcings that specialize higher Aronszajn trees. Fund. Math., 239(1):51-84, 2017.

[Mal96] Vyacheslav Ivanovich Malykhin. The Suslin hypothesis and its significance for settheoretic mathematics. Russian Mathematical Surveys, 51(3):419-437, 1996.

[Mil43] Edwin W. Miller. A note on Souslin's problem. Amer. J. Math., 65(4):673-678, 1943.

[Ost76] A. J. Ostaszewski. On countably compact, perfectly normal spaces. J. London Math. Soc. (2), 14(3):505-516, 1976.

[Rin11a] Assaf Rinot. Jensen's diamond principle and its relatives. In Set theory and its applications, volume 533 of Contemp. Math., pages 125-156. Amer. Math. Soc., Providence, RI, 2011.

[Rin11b] Assaf Rinot. On guessing generalized clubs at the successors of regulars. Ann. Pure Appl. Logic, 162(7):566-577, 2011.

[Rin14] Assaf Rinot. The Ostaszewski square, and homogeneous Souslin trees. Israel J. Math., 199(2):975-1012, 2014.

[Rin15] Assaf Rinot. Chromatic numbers of graphs - large gaps. Combinatorica, 35(2):215-233, 2015.

[Rin17] Assaf Rinot. Higher Souslin trees and the GCH, revisited. Adv. Math., 311(C):510-531, 2017.

[Rin19] Assaf Rinot. Souslin trees at successors of regular cardinals. Math. Log. Q., 65(2): 200204, 2019

[RZ20] Assaf Rinot and Jing Zhang. Strongest colorings. In preparation, 2020.

[Roi90] Judith Roitman. Introduction to modern set theory. Pure and Applied Mathematics (New York). John Wiley \& Sons, Inc., New York, 1990. A Wiley-Interscience Publication. 
[Rud69] Mary Ellen Rudin. Souslin's conjecture. Amer. Math. Monthly, 76(10):1113-1119, 1969.

[Sch14] Ralf Schindler. Set theory. Exploring independence and truth. Universitext. Springer, Cham, 2014.

[She84a] Saharon Shelah. An $\aleph_{2}$ Souslin tree from a strange hypothesis. Abs. Amer. Math. Soc., 160:198, 1984.

[She84b] Saharon Shelah. Can you take Solovay's inaccessible away? Israel Journal of Mathematics, 48:1-47, 1984 .

[She99] Saharon Shelah. On full Suslin trees. Colloquium Mathematicum, 79:1-7, 1999.

[SZ99] Saharon Shelah and Jindrich Zapletal. Canonical models for $\aleph_{1}$ combinatorics. Annals of Pure and Applied Logic, 98:217-259, 1999.

[ST71] R. M. Solovay and S. Tennenbaum. Iterated Cohen extensions and Souslin's problem. Ann. of Math. (2), 94(2):201-245, 1971.

[Sou20] Mikhail Yakovlevich Souslin. Problème 3. Fundamenta Mathematicae, 1(1):223, 1920.

[SS78] Lynn Arthur Steen and J. Arthur Seebach, Jr. Counterexamples in topology. SpringerVerlag, New York-Heidelberg, second edition, 1978.

[Ten68] Stanley Tennenbaum. Souslin's problem. Proc. Nat. Acad. Sci. U.S.A., 59(1):60-63, 1968.

[Tod84] Stevo Todorčević. Trees and linearly ordered sets. In Handbook of set-theoretic topology, pages 235-293. North-Holland, Amsterdam, 1984.

[Vel86] Boban Veličković. Jensen's $\square$ principles and the Novák number of partially ordered sets. J. Symbolic Logic, 51(1):47-58, 1986.

Department of Mathematics, Bar-Ilan University, RAMAT-Gan 5290002, Israel.

$U R L:$ http://u.math.biu.ac.il/ brodska/

$U R L:$ http://www. assafrinot.com 